Equações parciais elípticas com crescimento exponencial

Yony Raúl Santaria Leuyacc 


\title{
Equações parciais elípticas com crescimento exponencial
}

\author{
Yony Raúl Santaria Leuyacc
}

Orientador: Prof. Dr. Sérgio Henrique Monari Soares

Dissertação apresentada ao Instituto de Ciências Matemáticas e de Computação - ICMC-USP, como parte dos requisitos para obtenção do título de Mestre em Ciências - Matemática. VERSÃO REVISADA

USP - São Carlos

Março de 2014 
Ficha catalográfica elaborada pela Biblioteca Prof. Achille Bassi e Seção Técnica de Informática, ICMC/USP, com os dados fornecidos pelo(a) autor(a)

\begin{tabular}{|c|c|}
\hline \multirow[t]{3}{*}{ L652e } & $\begin{array}{l}\text { Leuyacc, Yony Raúl Santaria } \\
\text { Equações parciais elípticas com crescimento } \\
\text { exponencial / Yony Raúl Santaria Leuyacc; } \\
\text { orientador Sérgio Henrique Monari Soares. -- São } \\
\text { Carlos, } 2014 \text {. } \\
\text { 108 p. }\end{array}$ \\
\hline & $\begin{array}{l}\text { Dissertação (Mestrado - Programa de Pós-Graduação } \\
\text { em Matemática) -- Instituto de Ciências Matemáticas } \\
\text { e de Computação, Universidade de são Paulo, } 2014 .\end{array}$ \\
\hline & $\begin{array}{l}\text { 1. Desigualdades de Trudinger-Moser. 2. Equações } \\
\text { diferenciais parciais elipticas. 3. Crescimento } \\
\text { exponencial. 4. Métodos variacionais. I. Soares, } \\
\text { Sérgio Henrique Monari, orient. II. Tŕtulo. }\end{array}$ \\
\hline
\end{tabular}


A minha família. 


\section{Agradecimentos}

Agradeço especialmente ao professor Dr. Sérgio Monari a orientação, amizade e apoio. Agradeço-lhe os conhecimentos ensinados e a minha introdução ao fascinante mundo das EDPs elípticas.

Agradeço à Jackeline as palavras de motivação e o apoio constante para que continuasse meu trabalho com sucesso e para superar-me cada dia.

Agradeço aos meus amigos e professores da Universidad Nacional de San Marcos, em especial ao professor Agripino Garcia, principal incentivador para fazer o mestrado.

Agradeço ao povo brasileiro a calorosa receptividade em acolher-me de braços abertos neste lindo país.

Agradeço à Universidade de São Paulo, em particular ao Instituto de Matemáticas e Computação, que contribuiu para minha formação acadêmica.

Agradeço aos amigos que fiz nesta cidade, pela ajuda e por me fazer mais grato em minha estância.

Agradeço à CAPES - Coordenação de Aperfeiçoamento de Pessoal de Nível Superior o apoio financeiro e à coordenação da Pós Graduação em Matemática da USP. 

The greatest enemy of knowledge is not ignorance, it is the illusion of knowledge.

Stephen Hawking 


\section{Resumo}

Neste trabalho estudamos existência, multiplicidade e não existência de soluções não triviais para o seguinte problema elíptico:

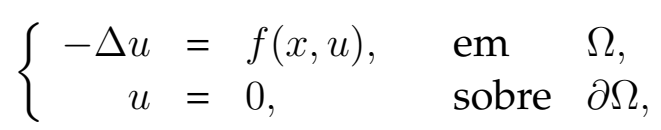

onde $\Omega$ é um conjunto limitado de $\mathbb{R}^{2}$ com fronteira suave e a função $f$ possui crescimento exponencial. Para a existência de soluções são aplicados métodos variacionais combinados com as desigualdades de Trudinger-Moser. O resultado de não-existência é restrito ao caso de soluções radiais positivas e $\Omega=B_{1}(0)$. A prova usa técnicas de equações diferenciais ordinárias.

Palavras-chave: Espaços de Orlicz, Desigualdades de Trudinger-Moser, Equações parciais elípticas, Crescimento exponencial, Métodos variacionais. 
viii 


\section{Abstract}

In this work we study the existence, multiplicity and non-existence of non-trivial solutions to the following elliptic problem:

$$
\left\{\begin{aligned}
-\Delta u & =f(x, u), & & \text { in } \Omega, \\
u & =0, & & \text { on } \partial \Omega,
\end{aligned}\right.
$$

where $\Omega$ is a bounded and smooth domain in $\mathbb{R}^{2}$ and $f$ possesses exponential growth. The existence results are proved by using variational methods and the TrudingerMoser inequalities. The non-existence result is restricted to the case of positive radial solutions and $\Omega=B_{1}(0)$. The proof uses techniques of the theory of ordinary differential equations.

Keywords: Orlicz spaces, Trudinger-Moser inequalities, Partial elliptic equations, Exponential growth, Variational methods. 


\section{Sumário}

Introdução

Notações $\quad 5$

1 Imersões nos Espaços de Orlicz $\quad 7$

1.1 Classes e espaços de Orlicz . . . . . . . . . . . . . . . . 7

1.2 Teoremas de imersão nos espaços de Orlicz . . . . . . . . . . . . . . . . 15

1.3 Otimalidade das imersões nos espaços de Orlicz . . . . . . . . . . . . 23

2 A desigualdade de Trudinger-Moser 27

2.1 Simetrização de Schwarz . . . . . . . . . . . . . . . . 27

2.2 Lemas . . . . . . . . . . . . . . . . . . . . . . . . 30

2.3 A desigualdade de Trudinger-Moser . . . . . . . . . . . . . . 35

3 Equações elípticas com crescimento crítico e subcrítico 43

3.1 A formulação variacional . . . . . . . . . . . . . . . . . 45

3.2 Demonstrações dos Teoremas . . . . . . . . . . . . . . . . 63

3.2.1 O caso subcrítico ..................... 63

3.2 .2 O caso crítico ........................... 65

4 Existência e não existência de soluções radiais $\quad 71$

4.1 Formulação do problema . . . . . . . . . . . . . . . 72

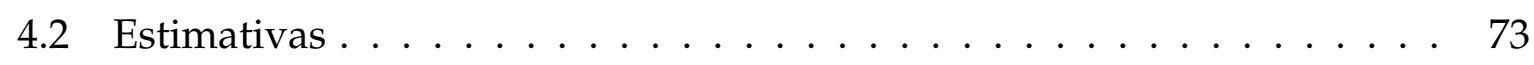

4.3 Demonstrações dos Teoremas . . . . . . . . . . . . . . . 86

A Resultados Básicos $\quad 95$

A.1 Os Espaços de Sobolev . . . . . . . . . . . . . . . . . . . . 96 
A.2 O Teorema do Passo da Montanha . . . . . . . . . . . . . . . . . 97

$\begin{array}{lll}\text { B Resultados técnicos } & 101\end{array}$ 


\section{Introdução}

Equações diferencias parciais elípticas com crescimento exponencial surgem naturalmente em processos físicos na forma de modelos matemáticos associados a problemas de reação de combustão. Em geometria Riemanniana, equações elípticas com crescimento exponencial estão presentes no estudo do problema de Yamabe a respeito da existência de uma métrica sobre uma variedade compacta de dimensão dois cuja curvatura é constante.

O objetivo deste trabalho é estudar existência, multiplicidade e não-existência de soluções para uma classe de problemas elípticos

$$
(P) \quad\left\{\begin{array}{clc}
-\Delta u=f(x, u), & \text { em } \quad \Omega, \\
u=0, & \text { sobre } & \partial \Omega
\end{array}\right.
$$

onde $\Omega \subset \mathbb{R}^{N}$ é um domínio limitado com fronteira $\partial \Omega$ suave e a $f: \bar{\Omega} \times \mathbb{R} \rightarrow \mathbb{R}$ é uma função satisfazendo a condição de crescimento: existe $\alpha_{0} \geq 0$ tal que

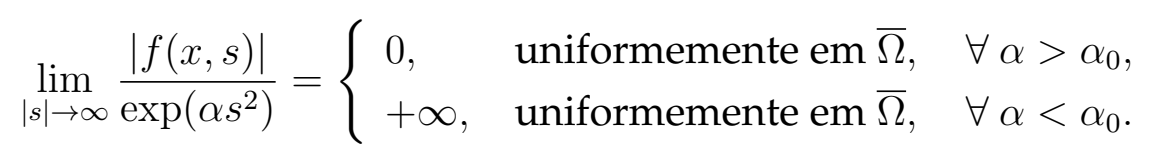

Na literatura [2, 8, 7], diz-se que $f(x, s)$ tem crescimento subcrítico ou crítico quando $\alpha_{0}=0$ ou $\alpha_{0}>0$, respectivamente.

Para motivar essa noção de criticalidade, observamos inicialmente que em dimensão dois, o teorema de imersão de Sobolev não fornece um crescimento crítico, pois $H_{0}^{1}(\Omega) \subset L^{q}(\Omega)$ para qualquer $q \geq 1$, mas $H_{0}^{1}(\Omega) \nsubseteq L^{\infty}(\Omega)$. Existe, porém, um crescimento crítico que é dado pelas desigualdades de Trudinger-Moser [11, 13], as quais estabelecem que para $0 \leq \alpha \leq 4 \pi$ existe uma constante $C>0$ independente de $\alpha$ tal que

$$
\int_{\Omega} e^{\alpha u^{2}} d x \leq C|\Omega|
$$


Além disso, Moser observou que a limitação $\alpha \leq 4 \pi$ é necessária e é a melhor possível (veja Capítulos 1 e 2 a seguir).

Em [6], Carleson e Chang provaram que a desigualdade em (1) é atingido no caso em que $\Omega$ é uma bola unitária do $\mathbb{R}^{2}$. Isto assegura a existência de uma constante $\lambda>0$ tal que a equação

$$
\left\{\begin{array}{clc}
-\Delta u=\lambda 8 \pi u e^{4 \pi u^{2}}, & \text { em } & \Omega \\
u=0, & \text { sobre } & \partial \Omega
\end{array}\right.
$$

possui uma solução positiva. Portanto, escrevendo a equação (2) na forma

$$
\left\{\begin{array}{cl}
-\Delta u=f(u), & \text { em } \Omega \\
u=0, & \text { sobre } \partial \Omega
\end{array}\right.
$$

o termo $f(u)$ satisfaz

$$
\lim _{|s| \rightarrow \infty} \frac{|f(s)|}{\exp \left(\alpha s^{2}\right)}= \begin{cases}0, & \forall \alpha>4 \pi, \\ +\infty, & \forall \alpha<4 \pi .\end{cases}
$$

Observamos que pela positividade da solução extremal, podemos escrever (2) na forma

$$
\left\{\begin{array}{cll}
-\Delta u=\lambda 8 \pi e^{4 \pi u^{2}+\ln u}, & \text { em } & \Omega, \\
u=0, & \text { sobre } & \partial \Omega,
\end{array}\right.
$$

ou seja, o termo $\ln u$ corresponde a uma perturbação de ordem inferior do termo $u^{2}$. Deste modo, ao considerar a equação mais geral

$$
\left(P_{1}\right) \quad\left\{\begin{array}{cll}
-\Delta u=e^{\alpha_{0} u^{2}+\ln h(u)}, & \text { em } & \Omega, \\
u=0, & \text { sobre } & \partial \Omega .
\end{array}\right.
$$

Em [3] Adimurthi e Yadava mostraram que uma condição suficiente, além de outras, para a existência de uma solução de $\left(P_{1}\right)$ é dado por:

$$
\lim _{|r| \rightarrow+\infty} h(r) r=+\infty
$$

Utilizando métodos variacionais, argumentos do tipo Passo da Montanha e a desigualdade de Trudinger-Moser, Figueiredo, Miyagaki e Ruf [7] obtiveram resultados de existência e de multiplicidade para o problema do tipo

$$
\left(P_{2}\right) \quad\left\{\begin{array}{cll}
-\Delta u=h(u, x) e^{\alpha_{0} u^{2}}, & \text { em } & \Omega, \\
u=0, & \text { sobre } & \partial \Omega .
\end{array}\right.
$$


Onde termo $h$ corresponde a uma perturbação de ordem inferior do termo $u^{2}$. Eles provaram a existência de solução para $\left(P_{2}\right)$ no caso crítico supondo que a existência de uma constante $K_{1}>0$ tal que

$$
\lim _{|t| \rightarrow+\infty} h(t, x) t>K_{1}=\frac{2}{\alpha_{0} d^{2}}
$$

onde $d$ é o raio da maior bola contida em $\Omega$. Estes resultados são apresentados no Capítulo 3.

Em [8], usando técnicas de equações diferenciais ordinárias, De Figueiredo e Ruf provaram que para $\Omega=B_{1}(0)$ e $\alpha_{0}=1$, existe de uma constante $K_{0}>0$ tal que se

$$
h(r)=\frac{K}{r} \text { para } r \text { suficientemente grande e } 0<K<K_{0}
$$

e $h$ satisfazendo algumas condições na origem, então a equação $\left(P_{1}\right)$ não possui solução radial positiva. Note que por [9], qualquer solução positiva de $\left(P_{1}\right)$ em $B_{1}(0)$ é radial; isto implica a não existência de solução positiva nestas condições. Este resultado é apresentado no Capítulo 4. 


\section{Notações}

1. $\mathbb{R}^{N}$ denota o Espaço euclidiano $\mathrm{N}$-dimensional. $N \geq 2$.

2. $\frac{\partial u}{\partial x_{i}}$ denota a derivada parcial da função $u$ em relação a $i$-ésima coordenada.

3. $\nabla u=\left(\frac{\partial u}{\partial x_{1}}, \frac{\partial u}{\partial x_{2}}, \ldots, \frac{\partial u}{\partial x_{n}}\right)$ denota o gradiente de $u$.

4. $\Delta u=\sum_{i=1}^{n} \frac{\partial^{2} u}{\partial x_{i}^{2}}$, denota o Laplaciano de $u$.

5. $\liminf _{n \rightarrow \infty} f_{n}$ denota o limite inferior da sequência $f_{n}$ quando $n \longrightarrow \infty$.

6. $|A|$ denota a medida de Lebesgue de um conjunto mensurável $A \subset \mathbb{R}^{N}$.

7. $B_{r}\left(x_{0}\right)$ denota bola aberta em $\mathbb{R}^{N}$, de centro $x_{0}$ e raio $r$.

8. $S^{N-1}$ denota a esfera unitária em $\mathbb{R}^{N}$.

9. $\omega_{N}$ é a medida $(N-1)$-dimensional de $S^{N-1}$.

10. $\{f<g\}$ denota o conjunto $\{x \in \Omega: f(x)<g(x)\}$ analogamente se define os conjuntos $\{f \leq g\},\{f>g\}$ e $\{f \geq g\}$.

11. $\mathcal{C}_{c}^{\infty}(\Omega)$ denota o espaço das funções infinitamente diferenciáveis $\varphi: \Omega \rightarrow \mathbb{R}$ com suporte compacto em $\Omega$.

12. $f(x)=O(g(x))$ denota que, existem $C>0$ e $x_{0}>0$ tal que $|f(x)| \leq C|g(x)|$ para todo $x \geq x_{0}$.

13. $f(x)=O_{+}(g(x))$ denota que, existe $C>0$ tal que $|f(x)| \leq C g(x)$, para todo $x$ no domínio . 


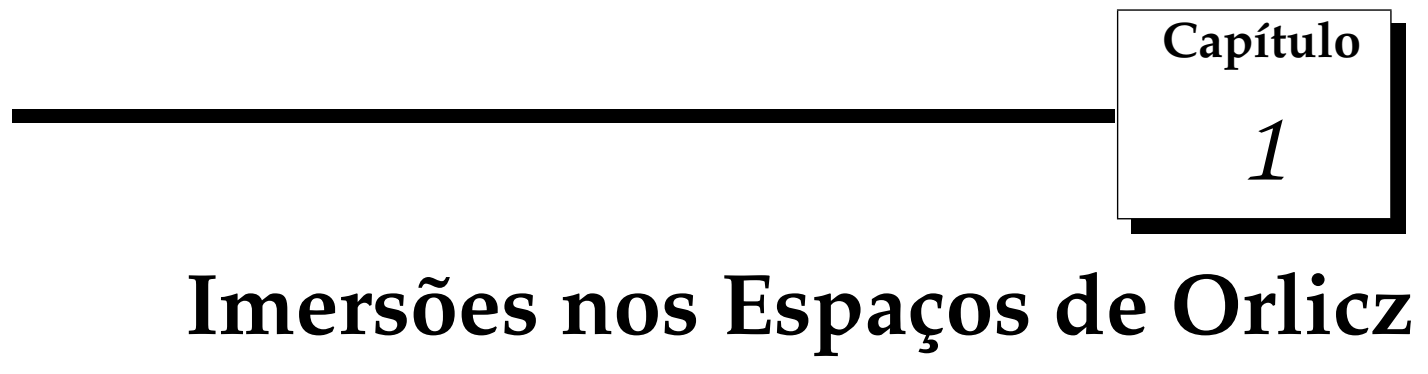

Neste capítulo introduzimos definições, conceitos e propriedades básicas dos espaços de Orlicz, os quais são uma generalização dos espaços $L^{p}$. Esta teoria foi proposta por Wladyslaw Orlicz em 1931. A ideia principal é substituir a função $\phi(t)=$ $|t|^{p}$ por outra positiva, convexa e crescente. Seguindo [13], apresentamos também os espaços de Morrey $L^{p, \lambda}(\Omega)$ que permitem a construção dos espaços $W^{k, p, \lambda}(\Omega)$, em particular $W^{k, p, N}(\Omega)=W^{k, p}(\Omega)$. Os resultados principais deste capítulo são relativos às imersões dos espaços $W^{k, p, \lambda}(\Omega)$ em certos espaços de Orlicz, onde $\Omega$ é um domínio limitado em $\mathbb{R}^{N}$ satisfazendo a condição de cone. Por último, mostramos que estas imersões são em um certo sentido ótimas.

\subsection{Classes e espaços de Orlicz}

Definição 1.1. Diz-se que uma função $u \in L^{p}(\Omega)$ pertence ao espaço de Morrey $L^{p, \lambda}(\Omega)$ se existe uma constante $K>0$ tal que

$$
\int_{\Omega \cap B}|u(x)|^{p} d x \leq K|B|^{1-\frac{\lambda}{N}}
$$

para qualquer bola $B$, onde $|B|$ denota a medida $N$-dimensional de $B$.

O espaço $L^{p, \lambda}(\Omega)$ é um espaço de Banach munido com a seguinte norma.

$$
\|u\|_{L^{p, \lambda}(\Omega)}:=\left(\sup _{B}|B|^{1-\frac{\lambda}{N}} \int_{\Omega \cap B}|u(x)|^{p} d x\right)^{\frac{1}{p}} .
$$


Em particular, temos

$$
L^{p, \lambda}(\Omega)=\left\{\begin{array}{cc}
\{0\}, & \lambda<0, \\
L^{\infty}(\Omega), & \lambda=0, \\
L^{p}(\Omega), & \lambda=N .
\end{array}\right.
$$

Definição 1.2. Definimos os seguintes espaços :

$$
W^{k, p, \lambda}(\Omega):=\left\{u \in W^{k, p}(\Omega) ; D^{\alpha}(u) \in L^{p, \lambda}(\Omega) \text {, para todo } \alpha \in \mathbb{N}^{N} \text { tal que }|\alpha|=k\right\}
$$

e

$$
W_{0}^{k, p, \lambda}(\Omega):=W_{0}^{k, p}(\Omega) \cap W^{k, p, \lambda}(\Omega),
$$

com a norma $\|u\|_{W^{k, p, \lambda}(\Omega)}:=\|u\|_{W^{k-1, p}(\Omega)}+\sum_{|\alpha|=k}\left\|D^{\alpha} u\right\|_{L^{p, \lambda}(\Omega)}$.

Definição 1.3. Uma função $\phi: \mathbb{R} \rightarrow \mathbb{R}$ contínua, convexa e par satisfazendo

$$
\lim _{t \rightarrow 0} \frac{\phi(t)}{t}=0, \quad \lim _{t \rightarrow \infty} \frac{\phi(t)}{t}=+\infty
$$

será chamada de N-função.

Exemplo 1.4. Como exemplo de N-função podemos citar

$$
\phi(t)=|t|^{p}, p>1, \quad \psi(t)=e^{|t|}-|t|-1, \quad \theta(t)=e^{|t|^{p}}-1, p>1
$$

Definição 1.5. Dada uma N-função $\phi$, a Classe de Orlicz $L^{\phi}(\Omega)$ é definido como o conjunto de todas as funções u tais que

$$
\int_{\Omega} \phi(u(x)) d x<+\infty
$$

Definição 1.6. Dada uma $N$-função $\phi$, o Espaço de Orlicz $L^{\phi^{*}}(\Omega)$ é definido como o espaço (das classes de equivalência) gerado por $L^{\phi}(\Omega)$ com a norma de Luxembourg

$$
\|u\|_{L^{\phi^{*}}(\Omega)}:=\inf \left\{k>0 ; \int_{\Omega} \phi\left(\frac{u}{k}\right) d x \leq 1\right\}
$$

onde ué equivalente a $v$ se, e só se $u=v$ q.t.p. em $\Omega$.

Quando o conjunto $\Omega$ estiver subentendido, denotaremos a norma $\|\cdot\|_{L^{\phi^{*}}(\Omega)}$ simplesmente por $\|\cdot\|_{L^{\phi^{*}}}$.

Sejam $\phi$ e $\psi$ N-funções. Se para todo $\lambda>0$ ocorrer

$$
\lim _{t \rightarrow \infty} \frac{\phi(\lambda t)}{\psi(t)}=+\infty
$$


escrevemos $\psi \prec \phi$, note que neste caso, temos $L^{\phi^{*}}(\Omega) \varsubsetneqq L^{\psi^{*}}(\Omega)$.

Lema 1.7. Para cada $u \in L^{\phi^{*}}(\Omega)$, tem-se

$$
\int_{\Omega} \phi\left(\frac{u(x)}{\|u\|_{L^{\phi^{*}}}}\right) d x \leq 1
$$

Demonstração. Seja $\left\{k_{n}\right\}$ uma sequência minimizante para $\|u\|_{L^{\phi^{*}}}$, isto é,

$$
\int_{\Omega} \phi\left(\frac{u(x)}{k_{n}}\right) d x \leq 1, \text { para cada } n \geq 1 \quad \text { e } \quad \lim _{n \rightarrow \infty} k_{n}=\|u\|_{L^{\phi^{*}}}
$$

Como $\phi$ é uma função contínua, temos

$$
\lim _{n \rightarrow \infty} \phi\left(\frac{u(x)}{k_{n}}\right)=\phi\left(\frac{u(x)}{\|u\|_{L^{\phi^{*}}}}\right), \text { para todo } x \in \Omega
$$

Pelo Lema de Fatou,

$$
\int_{\Omega} \phi\left(\frac{u(x)}{\|u\|_{L^{\phi^{*}}}}\right) d x \leq \liminf _{n \rightarrow \infty} \int_{\Omega} \phi\left(\frac{u(x)}{k_{n}}\right) d x \leq 1
$$

Teorema 1.8. Se $\phi$ é uma $N$-função, então $\|\cdot\|_{L^{\phi^{*}}(\Omega)}$ define uma noma. Além disso, o espaço $L^{\phi^{*}}(\Omega)$ munido com esta norma é um espaço de Banach.

Demonstração. Verificaremos que se satisfazem as condições de norma.

(i) Seja $u$, tal que $\|u\|_{L^{\phi^{*}}}=0$, logo para cada $k>0$, existe $\tilde{k}$ satisfazendo

$$
0<\tilde{k}<k \quad \text { e } \quad \int_{\Omega} \phi\left(\frac{u(x)}{\tilde{k}}\right) d x \leq 1
$$

Como $\phi$ é par é crescente em $[0, \infty)$, temos $\int_{\Omega} \phi\left(\frac{|u(x)|}{k}\right) d x \leq \int_{\Omega} \phi\left(\frac{|u(x)|}{\tilde{k}}\right) d x \leq 1$. Logo,

$$
\int_{\Omega} \phi\left(\frac{u(x)}{k}\right) d x \leq 1, \quad \text { para todo } k>0 .
$$

Suponha que existem $\epsilon>0$ e um conjunto $E \subset \Omega$ mensurável de medida positiva tais que $|u(x)|>\epsilon$, para todo $x \in E$. Logo

$$
\int_{\Omega} \phi\left(\frac{u(x)}{k}\right) d x \geq \int_{E} \phi\left(\frac{u(x)}{k}\right) d x \geq \int_{E} \phi\left(\frac{\epsilon}{k}\right) d x=|E| \phi\left(\frac{\epsilon}{k}\right) .
$$


Sendo $\phi$ uma N-função, temos $\lim _{k \rightarrow 0+} \phi\left(\frac{\epsilon}{k}\right)=+\infty$. Portanto, temos uma contradição com (1.3), consequentemente $u=0$, q.t.p. em $\Omega$.

Por outro lado, se $u=0$, q.t.p. em $\Omega$, se satisfaz (1.3), $\operatorname{logo},\|u\|_{L^{\phi^{*}}}=0$.

(ii) Seja $\alpha>0$, usando o Lema 1.7

$$
\int_{\Omega} \phi\left(\frac{(\alpha u)(x)}{\alpha\|u\|_{L^{\phi^{*}}}}\right) d x=\int_{\Omega} \phi\left(\frac{u(x)}{\|u\|_{L^{\phi^{*}}}}\right) d x \leq 1 .
$$

Portanto, $\|\alpha u\|_{L^{\phi^{*}}} \leq \alpha\|u\|_{L^{\phi^{*}}}$.

Pelo Lema 1.7

$$
\int_{\Omega} \phi\left(\frac{\alpha u(x)}{\|\alpha u\|_{L^{\phi^{*}}}}\right) d x \leq 1
$$

Assim, $\|u\|_{L^{\phi^{*}}} \leq \frac{\|\alpha u\|_{L^{\phi^{*}}}}{\alpha}$. Donde temos $\|\alpha u\|_{L^{\phi^{*}}}=\alpha\|u\|_{L^{\phi^{*}}}$, o caso $\alpha<0$ é análogo e o caso $\alpha=0$ é imediato.

(iii) Dados $u$ e $v$, tais que $\|u\|_{L^{\phi^{*}}} \mathrm{e}\|v\|_{L^{\phi^{*}}}$ são finitos. Seja

$$
\gamma=\|u\|_{L^{\phi^{*}}}+\|v\|_{L^{\phi^{*}}} ; \quad \alpha=\frac{\|u\|_{L^{\phi^{*}}}}{\gamma} ; \quad \beta=\frac{\|v\|_{L^{\phi^{*}}}}{\gamma} .
$$

Note que, $\alpha+\beta=1$. Usando a convexidade de $\phi$ e o Lema 1.7, obtemos

$$
\begin{aligned}
\int_{\Omega} \phi\left(\frac{u(x)+v(x)}{\gamma}\right) d x & =\int_{\Omega} \phi\left(\frac{\alpha u(x)}{\|u\|_{L^{\phi^{*}}}}+\frac{\beta v(x)}{\|v\|_{L^{\phi^{*}}}}\right) d x \\
& \leq \alpha \int_{\Omega} \phi\left(\frac{u(x)}{\|u\|_{L^{\phi^{*}}}}\right) d x+\beta \int_{\Omega} \phi\left(\frac{v(x)}{\|v\|_{L^{\phi^{*}}}}\right) d x \leq \alpha+\beta=1 .
\end{aligned}
$$

Donde, $\|u+v\|_{L^{\phi^{*}}} \leq \gamma=\|u\|_{L^{\phi^{*}}}+\|v\|_{L^{\phi^{*}}}$.

Consequentemente, $\|\cdot\|_{L^{\phi^{*}}(\Omega)}$ define uma norma em $L^{\phi^{*}}(\Omega)$.

Para ver que $L^{\phi^{*}}$ é um espaço de Banach com a norma $\|\cdot\|_{L^{\phi^{*}}(\Omega)}$ veja [10].

Definição 1.9. Diz-se que uma sequência $\left\{u_{n}\right\} \subset L^{\phi}(\Omega)$ converge em $L^{\phi}(\Omega)$ para $u \in L^{\phi^{*}}(\Omega)$ se

$$
\lim _{n \rightarrow \infty} \int_{\Omega} \phi\left(u_{n}(x)-u(x)\right) d x=0 .
$$

Observe que, para a N-função $\phi(t)=|t|^{p}$, com $p \geq 1$, tem-se a convergência em $L^{p}(\Omega)$. 
Definição 1.10. Denotemos por $E^{\phi}(\Omega)$ o conjunto $E^{\phi}(\Omega):={\overline{L^{\infty}(\Omega)}}^{\|\cdot\|_{L^{\phi^{*}}}}$.

Lema 1.11. A seguinte inclusão $E^{\phi}(\Omega) \subseteq L^{\phi}(\Omega)$ é valida para cada $N$-função $\phi$.

Demonstração. Seja $u \in E^{\phi}(\Omega)$, então existe $v \in L^{\infty}(\Omega)$, tal que $\|u-v\|_{L^{\phi^{*}}} \leq \frac{1}{2}$. Usando que $\phi$ é crescente no intervalo $[0,+\infty)$ e o Lema 1.7 , temos

$$
\int_{\Omega} \phi(2|u-v|) d x \leq \int_{\Omega} \phi\left(\frac{|u-v|}{\|u-v\|_{L^{\phi^{*}}}}\right) d x \leq 1 .
$$

Sendo $\phi$ é uma função par, então $2 u-2 v \in L^{\phi}(\Omega)$. Pela convexidade da função $\phi$, temos $\int_{\Omega} \phi(u) d x=\int_{\Omega} \phi\left(\frac{1}{2}(2 u-2 v)+\frac{1}{2}(2 v)\right) d x \leq \frac{1}{2} \int_{\Omega} \phi(2 u-2 v) d x+\frac{1}{2} \int_{\Omega} \phi(2 v) d x<+\infty$, pois,

$$
\int_{\Omega} \phi(2 v) d x \leq \sup _{0 \leq t \leq 2\|v\|_{L^{\infty}}} \phi(t)|\Omega|<+\infty
$$

E, portanto, $u \in L^{\phi}(\Omega)$.

Definição 1.12. Diz-se que um domínio $\Omega \subset \mathbb{R}^{N}$ satisfaz a condição do cone se existe um cone fixo $k_{\Omega}$ em $\mathbb{R}^{N}$ tal que cada ponto $x \in \Omega$ é o vértice de um cone $k_{\Omega}(x)$ congruente a $k_{\Omega}$ e contido em $\Omega$.

Lema 1.13. Sejam $\Omega \subset \mathbb{R}^{N}$ um domínio limitado satisfazendo a condição do cone e $u \in$ $W^{1,1}(\Omega)$. Então,

$$
|u(x)| \leq C\left(N, k_{\Omega}\right)\left(\int_{\Omega} \frac{|\nabla u(\xi)|}{|x-\xi|^{N-1}} d \xi+\|u\|_{L^{1}(\Omega)}\right), \quad \text { q.t.p. em } \Omega,
$$

onde $C\left(N, k_{\Omega}\right)$ é uma constante que só depende de $N$ e $k_{\Omega}$.

Demonstração. Consideremos primeiramente o caso em que $u \in \mathcal{C}^{\infty}(\Omega)$. Fize $x \in \Omega$ e para cada $y \in k_{\Omega}(x)$ considere a função $f(t)=u(y+t(x-y))$.

Pelo Teorema Fundamental do Cálculo temos, $f(1)-f(0)=\int_{0}^{1} f^{\prime}(t) d t$, isto é,

$$
u(x)-u(y)=\int_{0}^{1} \nabla u(y+t(x-y)) \cdot(x-y) d t, \quad \text { para todo } y \in k_{\Omega}(x) .
$$

Logo,

$$
|u(x)| \leq|u(y)|+\int_{0}^{1}|\nabla u(y+t(x-y))||(x-y)| d t, \quad \text { para todo } y \in k_{\Omega}(x) .
$$


Denotando por $k_{\Omega, r}(x):=k_{\Omega}(x) \cap B_{r}(x)$, onde $0<r \leq r_{\Omega}:=\operatorname{diam}\left(k_{\Omega}(x)\right)$.

Integrando (1.4) em relação a $y \in k_{\Omega, r}(x)$, temos

$$
\begin{aligned}
\left|k_{\Omega, r}(x) \| u(x)\right| & \leq \int_{k_{\Omega, r}(x)}|u(y)| d y+\int_{k_{\Omega, r}(x)} \int_{0}^{1}|\nabla u(y+t(x-y))||(x-y)| d t d y \\
& \leq\|u\|_{L^{1}(\Omega)}+\int_{0}^{1} \int_{K_{\Omega, r}(x)}|\nabla u(y+t(x-y)) \|(x-y)| d y d t .
\end{aligned}
$$

Da mudança de variável $\xi=y+t(x-y)$, temos $d \xi=(1-t)^{N} d y$ e $|x-y|=\frac{|\xi-x|}{(1-t)}$. Além disso, note que $y \in k_{\Omega, r}(x)$ se, e só se $\xi \in k_{\Omega,(1-t) r}(x)$. Assim

$$
\int_{0}^{1} \int_{k_{\Omega, r}(x)}|\nabla u(y+t(x-y)) \|(x-y)| d y d t=\int_{0}^{1} \int_{k_{\Omega,(1-t) r}(x)} \frac{\mid \nabla u(\xi))|| x-\xi \mid}{(1-t)^{N+1}} d \xi d t
$$

Usando o Teorema de Fubini na integral a direita em (1.6) e notando que

$$
\left\{(\xi, t) ; \xi \in k_{\Omega,(1-t) r}(x), 0 \leq t \leq 1\right\}=\left\{(\xi, t) ; 0 \leq t \leq 1-\frac{\xi-x}{r}, \xi \in k_{\Omega, r}(x)\right\},
$$

temos

$$
\begin{aligned}
\int_{0}^{1} \int_{k_{\Omega,(1-t) r}(x)} \frac{|\nabla u(\xi)||x-\xi|}{(1-t)^{N+1}} d \xi d t & =\int_{k_{\Omega, r}(x)}|\nabla u(\xi)||x-\xi| \int_{0}^{1-\frac{\xi-x}{r}} \frac{1}{(1-t)^{N+1}} d t d \xi \\
& =\int_{k_{\Omega, r}(x)}|\nabla u(\xi)||x-\xi|\left(\frac{\frac{r^{N}}{|\xi-x|^{N}}-1}{N}\right) d \xi \\
& \leq \int_{k_{\Omega, r}(x)}|\nabla u(\xi)||x-\xi|\left(\frac{r^{N}}{N|\xi-x|^{N}}\right) d \xi \\
& =\frac{r^{N}}{N} \int_{k_{\Omega, r}(x)} \frac{|\nabla u(\xi)|}{|x-\xi|^{N-1}} d \xi
\end{aligned}
$$

Usando (1.6) e (1.7) em (1.5), resulta

$$
\begin{aligned}
\left|k_{\Omega, r}(x) \| u(x)\right| & \leq\|u\|_{L^{1}(\Omega)}+\frac{r^{N}}{N} \int_{k_{\Omega, r}(x)} \frac{|\nabla u(\xi)|}{|x-\xi|^{N-1}} d \xi \\
& \leq\|u\|_{L^{1}(\Omega)}+\frac{r_{\Omega}^{N}}{N} \int_{K_{\Omega}(x)} \frac{|\nabla u(\xi)|}{|x-\xi|^{N-1}} d \xi
\end{aligned}
$$

Fazendo agora $r \rightarrow r_{\Omega}$ e lembrando que $\left|k_{\Omega, r_{\Omega}}(x)\right|=\left|k_{\Omega}(x)\right|=\left|k_{\Omega}\right|$, para cada $x \in \Omega$, 
temos

$$
\left|k_{\Omega}\right||u(x)| \leq\|u\|_{L^{1}(\Omega)}+\frac{r_{\Omega}^{N}}{N} \int_{K_{\Omega}(x)} \frac{|\nabla u(\xi)|}{|x-\xi|^{N-1}} d \xi
$$

E, portanto,

$$
|u(x)| \leq C\left(N, k_{\Omega}\right)\left(\int_{\Omega} \frac{|\nabla u(\xi)|}{|x-\xi|^{N-1}} d \xi+\|u\|_{L^{1}(\Omega)}\right), \quad \text { para todo } x \in \Omega
$$

onde $C\left(N, k_{\Omega}\right)=\max \left\{\frac{1}{\left|k_{\Omega}\right|}, \frac{r_{\Omega}^{N}}{N\left|k_{\Omega}\right|}\right\}$.

No caso geral, dada $u \in W^{1,1}(\Omega)$, existe uma sequência $\left\{u_{n}\right\} \subset \mathcal{C}^{\infty}(\Omega)$, tal que $u_{n} \rightarrow u$ em $W^{1,1}(\Omega)$, sem perda de generalidade podemos supor que:

$$
\nabla u_{n} \rightarrow \nabla u, \text { em } L^{1}(\Omega), \quad u_{n} \rightarrow u, \text { em } L^{1}(\Omega), \quad u_{n}(x) \rightarrow u(x) \text {, q.t.p. em } \Omega \text {. }
$$

Pela primeira parte da demonstração para funções em $\mathcal{C}^{\infty}(\Omega)$, temos para todo $n \geq 1$

$$
\left|u_{n}(x)\right| \leq C\left(N, k_{\Omega}\right)\left(\int_{\Omega} \frac{\left|\nabla u_{n}(\xi)\right|}{|x-\xi|^{N-1}} d \xi+\left\|u_{n}\right\|_{L^{1}(\Omega)}\right), \quad \text { para todo } x \in \Omega .
$$

De (1.8) e do Lema B.2 logo, podemos assumir que

$$
\int_{\Omega} \frac{\left|\nabla u_{n}(\xi)\right|}{|x-\xi|^{N-1}} d \xi \rightarrow \int_{\Omega} \frac{|\nabla u(\xi)|}{|x-\xi|^{N-1}} d \xi, \quad \text { q.t.p. em } \Omega \text {. }
$$

Logo, tomando o limite quando $n \rightarrow \infty$ em (1.9) e usando (1.8) , temos

$$
|u(x)| \leq C\left(N, k_{\Omega}\right)\left(\int_{\Omega} \frac{|\nabla u(\xi)|}{|x-\xi|^{N-1}} d \xi+\|u\|_{L^{1}(\Omega)}\right), \quad \text { q.t.p. em } \Omega .
$$

Lema 1.14. Seja $u \in W_{0}^{1,1}(\Omega)$, onde $\Omega \subset \mathbb{R}^{N}$ é um conjunto aberto e ilimitado, então

$$
|u(x)| \leq \frac{1}{N \omega_{N}} \int_{\Omega} \frac{|\nabla u(\xi)|}{|x-\xi|^{N-1}} d \xi, \quad \text { q.t.p. em } \Omega .
$$

Demonstração. Considere o caso tal que $u \in \mathcal{C}_{0}^{\infty}(\Omega)$. Dado $w \in S^{N-1}$, temos

$$
u(x)=-\int_{0}^{\infty} \frac{d(u(x+r w))}{d r} d r=-\int_{0}^{\infty} \nabla u(x+r w) \cdot w d r
$$


Logo, $|u(x)| \leq \int_{0}^{\infty}|\nabla u(x+r w)| d r$. Agora integrando em relação a $w \in S^{N-1}$, obtemos

$$
N \omega_{N}|u(x)| \leq \int_{S^{N-1}} \int_{0}^{\infty}|\nabla u(x+r w)| d r d w=\int_{S^{N-1}} \int_{0}^{\infty} \frac{|\nabla u(x+r w)|}{|x-(x+r w)|^{N-1}} r^{N-1} d r d w .
$$

Fazendo a mudança de variável $\xi=x+r w$ e usando coordenadas radiais na última integral, temos

$$
|u(x)| \leq \frac{1}{N \omega_{N}} \int_{\Omega} \frac{|\nabla u(\xi)|}{|x-\xi|^{N-1}} d \xi, \quad \text { para todo } x \in \Omega
$$

Para o caso geral, dado $u \in W_{0}^{1,1}(\Omega)$, existe uma sequência $\left\{u_{n}\right\} \subset \mathcal{C}_{0}^{\infty}(\Omega)$, tal que $u_{n} \rightarrow u$ em $W_{0}^{1,1}(\Omega)$, e faça a prova neste caso como na prova do Lema 1.13.

Lema 1.15. Sejam $\Omega \subset \mathbb{R}^{N}$ um conjunto limitado e convexo e $u \in W^{1,1}(\Omega)$. Defina

$$
u_{\Omega}=\frac{1}{|\Omega|} \int_{\Omega} u(x) d x
$$

Então

$$
\left\|u-u_{\Omega}\right\|_{L^{1}(\Omega)} \leq C(N)|\Omega|^{\frac{1}{N}} \frac{\operatorname{diam}(\Omega)^{N}}{|\Omega|}\|\nabla u\|_{L^{1}(\Omega)}
$$

Demonstração. Pela densidade, basta provar para $u \in \mathcal{C}^{\infty}(\Omega)$. Fixe $x \in \Omega$ e considere $y \in \Omega$ um ponto qualquer. Pela convexidade de $\Omega$, temos

$$
u(x)-u(y)=\int_{0}^{1} \nabla u(y+t(x-y)) \cdot(x-y) d t .
$$

Integrando em relação a $y \in \Omega$, temos

$$
|\Omega| u(x)-\int_{\Omega} u(y) d y=\int_{\Omega} \int_{0}^{1} \nabla u(y+t(x-y)) \cdot(x-y) d t d y
$$

isto é,

$$
|\Omega|\left(u(x)-u_{\Omega}\right)=\int_{\Omega} \int_{0}^{1} \nabla u(y+t(x-y)) \cdot(x-y) d t d y .
$$

Fazendo como na prova do Lema 1.13, temos

$$
\int_{\Omega} \int_{0}^{1} \nabla u(y+t(x-y)) \cdot(x-y) d t d y \leq \frac{\operatorname{diam}(\Omega)^{N}}{N} \int_{\Omega} \frac{|\nabla u(\xi)|}{|x-\xi|^{N-1}} d \xi
$$


Logo,

$$
\left|u(x)-u_{\Omega}\right| \leq \frac{\operatorname{diam}(\Omega)^{N}}{N|\Omega|} \int_{\Omega} \frac{|\nabla u(\xi)|}{|x-\xi|^{N-1}} d \xi, \quad \text { se } x \in \Omega .
$$

Integrando em relação a $x \in \Omega$ e usando o Lema B.1, temos

$$
\begin{aligned}
\left\|u-u_{\Omega}\right\|_{L^{1}(\Omega)} & =\int_{\Omega}\left|u(x)-u_{\Omega}\right| d x \leq \frac{\operatorname{diam}(\Omega)^{N}}{N|\Omega|} \int_{\Omega} \int_{\Omega} \frac{|\nabla u(\xi)|}{|x-\xi|^{N-1}} d \xi d x \\
& \leq \frac{\operatorname{diam}(\Omega)^{N}}{N|\Omega|} \int_{\Omega}|\nabla u(\xi)| \int_{\Omega} \frac{1}{|x-\xi|^{N-1}} d x d \xi \\
& \leq \frac{\operatorname{diam}(\Omega)^{N}}{N|\Omega|} C_{0}(N)|\Omega|^{1 / N} \int_{\Omega}|\nabla u(\xi)| d \xi \\
& \leq C(N)|\Omega|^{1 / n} \frac{\operatorname{diam}(\Omega)^{n}}{|\Omega|} \mid \nabla u \|_{L^{1}(\Omega)},
\end{aligned}
$$

onde $C(N)=\frac{C_{0}(N)}{N}$.

\subsection{Teoremas de imersão nos espaços de Orlicz}

Teorema 1.16. Seja $\Omega \subset \mathbb{R}^{N}$ um conjunto limitado satisfazendo a condição do cone. Então o espaço $W^{1,1,1}(\Omega)$ está imerso continuamente no espaço de Orlicz $L^{\phi^{*}}(\Omega)$, onde $\phi(t)=$ $e^{|t|}-|t|-1$.

Além disso, para cada $u \in W^{1,1,1}(\Omega)$, temos

$$
\|u\|_{L_{\phi^{*}(\Omega)}} \leq C\left(N, k_{\Omega}, \operatorname{diam}(\Omega)\right)\|u\|_{W^{1,1,1}(\Omega)} .
$$

Demonstração. Seja $u \in W^{1,1,1}(\Omega)$, denotamos

$$
K=\|u\|_{W^{1,1,1}(\Omega)} ; \quad d=\operatorname{diam}(\Omega) ; \quad b=\frac{1}{K C}
$$

onde $C>0$ é uma constante apropriada a ser determinada. Considere $f \in L^{p}(\Omega)$, com $p>1$ e $q$ tal que $\frac{1}{p}+\frac{1}{q}=1$. Pelo Lema 1.13 existe uma constante $C_{1}=C_{1}\left(N, k_{\Omega}\right)$, tal que

$$
\begin{aligned}
\int_{\Omega}|f(x) u(x)| d x & \leq \int_{\Omega}|f(x)|\left(C_{1}\left(\int_{\Omega} \frac{|\nabla u(\xi)|}{|\xi-x|^{N-1}} d \xi+\|u\|_{L^{1}(\Omega)}\right)\right) d x \\
& \leq C_{1}\left(\int_{\Omega} \int_{\Omega} \frac{|f(x) \nabla(\xi)|}{|x-\xi|^{N-1}} d \xi d x+K \int_{\Omega}|f(x)| d x\right) \\
& =C_{1}\left(\int_{\Omega} \int_{\Omega}\left(\frac{|\nabla u(\xi)|}{|x-\xi|^{N-\frac{1}{q}}}\right)^{\frac{1}{q}}\left(\frac{|f(x)|^{p}|\nabla u(\xi)|}{|x-\xi|^{N-1-\frac{1}{q}}}\right)^{\frac{1}{p}} d \xi d x+K \int_{\Omega}|f(x)| d x\right)
\end{aligned}
$$




$$
\begin{aligned}
\leq & C_{1}\left(\int_{\Omega} \int_{\Omega} \frac{|\nabla u(\xi)|}{|x-\xi|^{N-\frac{1}{q}}} d \xi d x\right)^{\frac{1}{q}}\left(\int_{\Omega} \int_{\Omega} \frac{|f(x)|^{p}|\nabla u(\xi)|}{|x-\xi|^{N-1-\frac{1}{q}}} d \xi d x\right)^{\frac{1}{p}}+ \\
& +C_{1} K \int_{\Omega}|f(x)| d x
\end{aligned}
$$

Vamos agora estimar cada uma dessas integrais em (1.11).

- Estimativa para $\int_{\Omega} \int_{\Omega} \frac{|\nabla u(\xi)|}{|x-\xi|^{N-\frac{1}{q}}} d \xi d x$

Pelo Lema B.1, podemos encontrar uma constante $C_{2}=C_{2}(N)$, tal que

$$
\int_{\Omega} \frac{1}{|x-\xi|^{N-\frac{1}{q}}} d x \leq C_{2} q|\Omega|^{\frac{1}{q N}} .
$$

Por outro lado, como $u \in W^{1,1,1}(\Omega),|\nabla u| \in L^{1,1}(\Omega)$, de (1.1), para a bola $B=B_{d}\left(x_{o}\right)$, com $x_{0}$ fixo em $\Omega$, resulta

$$
\int_{\Omega \cap B_{d}\left(x_{o}\right)}|\nabla u(\xi)| d \xi \leq\|\nabla u\|_{L^{1,1}(\Omega)}\left|B_{d}\left(y_{o}\right)\right|^{1-\frac{1}{N}} \leq K\left|B_{d}\left(x_{o}\right)\right|^{1-\frac{1}{N}}
$$

Como $\Omega \subseteq B_{d}\left(x_{o}\right),|\Omega| \leq\left|B_{d}\left(x_{o}\right)\right|=C_{3} d^{N}, \operatorname{com} C_{3}=\omega_{N}$. Logo, de (1.12) e (1.13), tem-se

$$
\begin{aligned}
\int_{\Omega} \int_{\Omega} \frac{|\nabla u(\xi)|}{|x-\xi|^{N-\frac{1}{q}}} d \xi d x & =\int_{\Omega}|\nabla u(\xi)| \int_{\Omega} \frac{1}{|x-\xi|^{N-\frac{1}{q}}} d \xi d x \\
& \leq q C_{2}|\Omega|^{\frac{1}{q N}} \int_{\Omega}|\nabla u(\xi)| d \xi \\
& \leq K q C_{2}|\Omega|^{\frac{1}{q N}}\left|B_{d}\left(x_{o}\right)\right|^{1-\frac{1}{N}} \\
& \leq K q C_{2}\left(C_{3} d^{N}\right)^{\frac{1}{q N}}\left(C_{3} d^{N}\right)^{1-\frac{1}{N}}=C_{4} K q d^{N-1+\frac{1}{q}}
\end{aligned}
$$

onde $C_{4}=C_{4}(N)$.

- Estimativa para $\int_{\Omega} \int_{\Omega} \frac{|f(x)|^{p}|\nabla u(\xi)|}{|x-\xi|^{N-1-\frac{1}{q}}} d \xi d x$

Para cada $x \in \Omega$ e $\rho>0$, defina a função $v_{x}(\rho)=\int_{\Omega \cap B_{\rho}(x)}|\nabla u(\xi)| d \xi$. Logo, de (1.1),

$$
v_{x}(\rho)=\int_{\Omega \cap B_{\rho}(x)}|\nabla u(\xi)| d \xi \leq K\left|B_{\rho}(x)\right|^{1-\frac{1}{N}}=K\left(C_{3} \rho^{N}\right)^{1-\frac{1}{N}} \leq K C_{5} \rho^{N-1},
$$

onde $C_{5}=C_{5}(N)$. Por outro lado, $v_{x}^{\prime}(\rho)=\int_{\Omega \cap S_{\rho}(x)}|\nabla u(\xi)| d \xi$. 
E, portanto,

$$
\frac{v_{x}^{\prime}(\rho)}{\rho^{N-1-\frac{1}{q}}}=\frac{1}{\rho^{N-1-\frac{1}{q}}} \int_{\Omega \cap S_{\rho}(x)}|\nabla u(\xi)| d \xi=\int_{\Omega \cap S_{\rho}(x)} \frac{|\nabla u(\xi)|}{|x-\xi|^{N-1-\frac{1}{q}}} d \xi .
$$

Por conseguinte, desta última igualdade e de (1.14), temos que

$$
\begin{aligned}
\int_{\Omega} \frac{|\nabla u(y)|}{|y-x|^{N-1-\frac{1}{q}}} d y \leq & \int_{0}^{d} \int_{\Omega \cap S_{\rho(x)}} \frac{|\nabla u(\xi)|}{|\xi-x|^{N-1-\frac{1}{q}}} d \xi d \rho=\int_{0}^{d} \frac{v_{x}^{\prime}(\rho)}{\rho^{N-1-\frac{1}{q}}} d \rho \\
= & \lim _{t \rightarrow 0} \int_{t}^{d} \frac{v_{x}^{\prime}(\rho)}{\rho^{N-1-\frac{1}{q}}} d \rho=\left.\lim _{t \rightarrow 0} \frac{v_{x}(\rho)}{\rho^{N-1-\frac{1}{q}}}\right|_{\rho=t} ^{\rho=d} \\
& -\left(-N+1+\frac{1}{q}\right) \int_{0}^{d} \frac{v_{x}(\rho)}{\rho^{N-\frac{1}{q}}} d \rho \\
\leq & \frac{v_{x}(d)}{d^{N-1-\frac{1}{q}}}-\lim _{\rho \rightarrow 0} \frac{v_{x}(\rho)}{\rho^{N-1-\frac{1}{q}}}+(N-1) \int_{0}^{d} \frac{v_{x}(\rho)}{\rho^{N-\frac{1}{q}}} d \rho \\
\leq & \frac{K C_{5} d^{N-1}}{d^{N-1-\frac{1}{q}}}+(N-1) \int_{0}^{d} \frac{K C_{5} \rho^{N-1}}{\rho^{N-\frac{1}{q}}} d \rho \\
\leq & K C_{5} d^{\frac{1}{q}}(1+q(N-1)),
\end{aligned}
$$

onde, usamos (1.14), para ter $\lim _{\rho \rightarrow 0} \frac{v_{x}(\rho)}{\rho^{N-1-\frac{1}{q}}}=0$. Logo,

$$
\begin{aligned}
\int_{\Omega} \int_{\Omega} \frac{|f(x)|^{p}|\nabla u(\xi)|}{|x-\xi|^{N-1-\frac{1}{q}}} d \xi d x & =\int_{\Omega}|f(x)|^{p} \int_{\Omega} \frac{|\nabla u(\xi)|}{|x-\xi|^{N-1-\frac{1}{q}}} d \xi d x \\
& \leq K C_{5} d^{\frac{1}{q}}(1+q(N-1))\|f\|_{L^{p}(\Omega)}^{p}
\end{aligned}
$$

E, portanto, das estimativas das integrais em (1.11), segue que

$$
\begin{aligned}
\int_{\Omega}|f(x) u(x)| d x & \leq C_{1}\left(C_{4} K q d^{N-1+\frac{1}{q}}\right)^{\frac{1}{q}}\left(K C_{5} d^{\frac{1}{q}}(1+q(N-1))\|f\|_{L^{p}(\Omega)}^{p}\right)^{\frac{1}{p}}+C_{1} K \int_{\Omega}|f(x)| d x \\
& =C_{1} C_{4}^{\frac{1}{q}} C_{5}^{\frac{1}{q}} K d^{\frac{N}{q}}(N q-q+1)^{\frac{1}{p}} q^{\frac{1}{q}}\|f\|_{L^{p}(\Omega)}+C_{1} K \int_{\Omega}|f(x)| d x \\
& \leq C_{7} K d^{\frac{N}{q}} q\|f\|_{L^{p}(\Omega)}+C_{1} K\|f\|_{L^{1}(\Omega)}, \quad \text { onde } C_{7}=C_{7}\left(N, k_{\Omega}\right) .
\end{aligned}
$$

Logo, para cada $q>1$ e $p>1$ tal que $\frac{1}{p}+\frac{1}{q}=1$

$$
\|u\|_{L^{q}(\Omega)}=\sup _{f \in L^{p}(\Omega) \backslash\{0\}} \frac{\int_{\Omega}|f(x) u(x)| d x}{\|f\|_{L^{p}(\Omega)}}
$$




$$
\begin{aligned}
& \leq \sup _{f \in L^{p}(\Omega) \backslash\{0\}} \frac{C_{7} K d^{\frac{N}{q}} q\|f\|_{L^{p}(\Omega)}+C_{1} K\|f\|_{L^{1}(\Omega)}}{\|f\|_{L^{p}(\Omega)}} \\
& \leq \sup _{f \in L^{p}(\Omega) \backslash\{0\}} \frac{C_{7} K d^{\frac{N}{q}} q\|f\|_{L^{p}(\Omega)}+C_{1} K\|f\|_{L^{p}(\Omega)}\|1\|_{L^{q}(\Omega)}}{\|f\|_{L^{p}(\Omega)}} \\
& =C_{7} K d^{\frac{N}{q}} q+C_{1} K|\Omega|^{\frac{1}{q}} \leq C_{7} K d^{\frac{N}{q}} q+C_{1} K C_{3}^{\frac{1}{q}} d^{\frac{N}{q}} \\
& \leq C_{8} K q d^{\frac{N}{q}}, \quad \text { onde } C_{8}=C_{8}\left(N, k_{\Omega}\right) .
\end{aligned}
$$

Logo, para qualquer $b>0$, temos

$$
\begin{aligned}
\int_{\Omega} \sum_{j=2}^{m} \frac{(b|u|)^{j}}{j !} d x & =\sum_{j=2}^{m} \frac{b^{j}}{j !} \int_{\Omega}|u|^{j} d x=\sum_{j=2}^{m} \frac{b^{j}}{j !}\|u\|_{L^{j}(\Omega)}^{j} \\
& \leq \sum_{j=2}^{m} \frac{b^{j}}{j !}\left(C_{8} K j d^{\frac{N}{j}}\right)^{j}=d^{N} \sum_{j=2}^{m} \frac{\left(C_{8} K b j\right)^{j}}{j !}
\end{aligned}
$$

Pelo Teste da Razão, $\sum_{j=1}^{+\infty} \frac{(a j)^{j}}{j !}$ converge se $|a|<\frac{1}{e}$. Portanto, escolhendo $C$ suficientemente grande de modo que

$$
0<b<\frac{1}{e C_{8} K} ; \quad \sum_{j=2}^{\infty} \frac{\left(C_{8} K b j\right)^{j}}{j !} \leq \frac{1}{d^{N}}
$$

Concluímos que,

$$
\begin{aligned}
\int_{\Omega}\left(e^{b|u|}-b|u|-1\right) d x & =\int_{\Omega} \lim _{m \rightarrow \infty} \sum_{j=2}^{m} \frac{(b|u|)^{j}}{j !} d x \\
& =\lim _{m \rightarrow \infty} d^{N} \sum_{j=2}^{m} \frac{\left(C_{8} K b j\right)^{j}}{j !} \leq 1 .
\end{aligned}
$$

Logo, da definição de $\phi$ e $b$, temos

$$
\int_{\Omega} \phi\left(\frac{u}{C\|u\|_{W^{1,1,1}(\Omega)}}\right) d x \leq 1
$$

E, portanto,

$$
\|u\|_{L^{\phi^{*}}} \leq C\left(N, k_{\Omega}, \operatorname{diam}(\Omega)\right)\|u\|_{W^{1,1,1}(\Omega)} .
$$

Corolário 1.17. Seja $\Omega$ um conjunto convexo limitado e suponha que $u \in W^{1,1}(\Omega)$ e 
$|\nabla u| \in L^{1,1}(\Omega)$, então existem constastes positivas $A$ e $B$, que só dependem de $N$ e $\Omega$, tais que

$$
\int_{\Omega}\left(e^{A \frac{\left|u(x)-u_{\Omega}\right|}{K}}-1\right) d x \leq B, \quad \text { onde } K=\|\nabla u\|_{L^{1,1}(\Omega)} .
$$

Demonstração. Defina $v(x)=u(x)-u_{\Omega}$, para $x \in \Omega$. Então $v \in W^{1,1}(\Omega)$ e $\nabla v=\nabla u$.

De (1.10), existe uma constante $C_{1}=C_{1}(N, \Omega)$ tal que

$$
|v(x)| \leq C_{1} \int_{\Omega} \frac{|\nabla v(\xi)|}{|x-\xi|^{N-1}} d \xi, \quad \text { q.t.p. em } \Omega
$$

Note que, usando a desigualdade acima e $K=\|\nabla u\|_{L^{1,1}(\Omega)}$ como na demonstração do Teorema 1.16, existe uma constante $C=C(N, \Omega)$ tal que

$$
\int_{\Omega} \phi\left(\frac{v(x)}{C K}\right) d x \leq 1
$$

Isto é,

$$
\int_{\Omega}\left(e^{\frac{\left|u(x)-u_{\Omega}\right|}{C K}}-\frac{\left|u(x)-u_{\Omega}\right|}{K}-1\right) d x \leq 1 .
$$

Além disso, pelo Lema 1.15, existe $C_{2}=C_{2}(N, \Omega)$ tal que $\left\|u(x)-u_{\Omega}\right\|_{L^{1}(\Omega)} \leq C_{2} K$.

E, portanto,

$$
\int_{\Omega}\left(e^{\frac{\left|u(x)-u_{\Omega}\right|}{C K}}-1\right) d x \leq 1+C_{2}
$$

Logo, para concluir a demonstração, basta tomar $A=\frac{1}{C}$ e $B=1+C_{2}$.

Lema 1.18. Seja $\Omega$ um domínio aberto limitado regular em $\mathbb{R}^{N}$, então a seguinte imersão é contínua

$$
W^{k, p}(\Omega) \hookrightarrow W^{1, N}(\Omega), \quad \text { se } N=k p
$$

Demonstração. Temos a seguintes imersões continuas :

Por $($ ii) do Teorema A.7, temos que

$$
W^{k, p}(\Omega) \hookrightarrow L^{N}(\Omega)
$$

Por $(i)$ do Teorema A.7, temos que

$$
W^{k-1, p}(\Omega) \hookrightarrow L^{N}(\Omega), \quad \text { pois } N=\frac{N p}{N-(k-1) p} .
$$

Assim, dada $u \in W^{k, p}(\Omega) \subset L^{N}(\Omega)$, tem-se $|\nabla u| \in W^{k-1, p}(\Omega) \subset L^{N}(\Omega)$. Portanto, $u \in L^{N}(\Omega)$ e $\nabla u \in L^{N}(\Omega)$. Ou seja $u \in W^{1, N}(\Omega)$. 
Agora estamos prontos para enunciar o resultado principal deste capítulo.

Teorema 1.19. Seja $\Omega \subset \mathbb{R}^{N}$, um conjunto limitado satisfazendo a condição de cone, então o espaço $W^{k, p}(\Omega)$, onde $N=k p$, está imerso continuamente no Espaço de Orlicz $L^{\phi^{*}}(\Omega)$, onde

$$
\phi(t)=e^{|t|^{N-1}}-1
$$

Além disso, $W^{k, p}(\Omega)$ está imerso continuamente no sentido na convergência $L^{\psi}(\Omega)$ em qualquer classe de Orlicz $L^{\psi}(\Omega)$, onde $\psi(t) \leq \phi(\lambda t)$, para algum $\lambda>0$.

Demonstração. Considere $f \in L^{p}(\Omega)$ com $p>1$ e $q$ tal que $\frac{1}{p}+\frac{1}{q}=1$. Pelo Lema 1.13 e a desigualdade de Hölder, tem-se

$$
\begin{aligned}
\int_{\Omega}|f(x) u(x)| d x \leq & \int_{\Omega}|f(x)|\left(C\left(N, k_{\Omega}\right)\left(\int_{\Omega} \frac{|\nabla u(\xi)|}{|\xi-x|^{N-1}} d \xi+\|u\|_{L^{1}(\Omega)}\right)\right) d x \\
\leq & C\left(\int_{\Omega} \int_{\Omega} \frac{|f(x) \nabla(\xi)|}{|x-\xi|^{N-1}} d \xi d x+\|u\|_{L^{1}(\Omega)} \int_{\Omega}|f(x)| d x\right) \\
= & C\left(\int_{\Omega} \int_{\Omega}\left(\frac{|f(x)|}{|x-\xi|^{N-\frac{1}{q}}}\right)^{1-\frac{1}{N}}\left(\frac{|\nabla u(\xi)|^{N}}{|x-\xi|^{\frac{N-1}{q}}}\right)^{\frac{1}{N}} d \xi d x+\|u\|_{L^{1}(\Omega)} \int_{\Omega}|f(x)| d x\right) \\
\leq & C\left(\int_{\Omega} \int_{\Omega} \frac{|f(x)|}{|x-\xi|^{N-\frac{1}{q}}} d \xi d x\right)^{1-\frac{1}{N}}\left(\int_{\Omega} \int_{\Omega} \frac{|\nabla u(\xi)|^{N}|f(x)|}{|x-\xi|^{\frac{N-1}{q}}} d \xi d x\right)^{\frac{1}{N}}+ \\
& +C\left|u \|_{L^{1}(\Omega)} \int_{\Omega}\right| f(x) \mid d x .
\end{aligned}
$$

Agora estimemos as integrais acima.

- Estimativa para $\int_{\Omega} \int_{\Omega} \frac{|f(x)|}{|x-\xi|^{N-\frac{1}{q}}} d \xi d x$.

Pelo Lema B.1, temos

$$
\begin{aligned}
\int_{\Omega} \int_{\Omega} \frac{|f(x)|}{|x-\xi|^{N-\frac{1}{q}}} d \xi d x & =\int_{\Omega}|f(x)| \int_{\Omega} \frac{1}{|x-\xi|^{N-\frac{1}{q}}} d \xi d x \leq\left. C_{1} q\right|^{\frac{1}{q N}} \int_{\Omega}|f(x)| d x \\
& \leq C_{1} q|\Omega|^{\frac{1}{q N}}\|f\|_{L^{p}(\Omega)}\|1\|_{L^{q}(\Omega)}=\left.C_{1} q\right|^{||^{\frac{1}{q N}\left(\frac{1}{N}+1\right)}\|f\|_{L^{p}(\Omega)} .}
\end{aligned}
$$

- Estimativa para $\int_{\Omega} \int_{\Omega} \frac{|\nabla u(\xi)|^{N}|f(x)|}{|x-\xi|^{\frac{N-1}{q}}} d \xi d x$. 
Pelo Teorema de Fubini e o Lema B.1, temos

$$
\begin{aligned}
\int_{\Omega} \int_{\Omega} \frac{|\nabla u(\xi)|^{N}|f(x)|}{|x-\xi|^{\frac{N-1}{q}}} d \xi d x & =\int_{\Omega}|\nabla u(\xi)|^{N} \int_{\Omega} \frac{|f(x)|}{|x-\xi|^{\frac{N-1}{q}}} d \xi d x \\
& \leq \int_{\Omega}|\nabla u(\xi)|^{N}\|f\|_{L^{p}(\Omega)}\left(\int_{\Omega} \frac{1}{|x-\xi|^{N-1}} d \xi\right)^{\frac{1}{q}} d x \\
& \leq C_{2}|\Omega|^{\frac{1}{q N}}\|f\|_{L^{p}(\Omega)}|\Omega|^{\frac{1}{q N}} \int_{\Omega}|\nabla u(\xi)|^{N} d x \\
& =C_{2}|\Omega|^{\frac{1}{q N}}\|f\|_{L^{p}(\Omega)}\|\nabla u\|_{L^{N}(\Omega)}^{N} .
\end{aligned}
$$

Logo, substituindo as estimativas das integrais em (1.16), resulta

$$
\begin{aligned}
\int_{\Omega}|f(x) u(x)| d x \leq & C\left(C_{1} q|\Omega|^{\frac{1}{q N}\left(\frac{1}{N}+1\right)}\|f\|_{L^{p}(\Omega)}\right)^{1-\frac{1}{N}}\left(C_{2}|\Omega|^{\frac{1}{q N}}\|f\|_{L^{p}(\Omega)}\|\nabla u\|_{L^{N}(\Omega)}^{N}\right)^{\frac{1}{N}} \\
& +C\|u\|_{L^{1}(\Omega)}\|f\|_{L^{P}(\Omega)}\|1\|_{L^{q}(\Omega)} \\
= & C\|f\|_{L^{p}(\Omega)}|\Omega|^{\frac{1}{q}}\left(C_{3} q^{\frac{N-1}{N}}\|\nabla u\|_{L^{N}(\Omega)}+\|u\|_{L^{1}(\Omega)}\right) \\
\leq & C_{4}\|f\|_{L^{p}(\Omega)}|\Omega|^{\frac{1}{q}} q^{\frac{N-1}{N}}\|u\|_{W^{1, N}(\Omega)}, \quad \text { onde } C_{4}=C_{4}\left(N, \Omega, k_{\Omega}\right) .
\end{aligned}
$$

Portanto, para cada $q>1$,

$$
\begin{aligned}
\|u\|_{L^{q}(\Omega)} & =\sup _{f \in L^{p}(\Omega) \backslash\{0\}} \frac{\int_{\Omega}|f(x) u(x)| d x}{\|f\|_{L^{p}(\Omega)}} \\
& \leq \sup _{f \in L^{p}(\Omega) \backslash\{0\}} \frac{C_{4}\|f\|_{L^{p}(\Omega)}|\Omega|^{\frac{1}{q}} q^{\frac{N-1}{N}}\|u\|_{W^{1, N}(\Omega)}}{\|f\|_{L^{p}(\Omega)}} \\
& =C_{4}|\Omega|^{\frac{1}{q}} q^{\frac{N-1}{N}}\|u\|_{W^{1, N}(\Omega)} .
\end{aligned}
$$

Usando (1.17) para $q=\frac{N j}{N-1}, \operatorname{com} j \geq 1$,

$$
\left(\int_{\Omega}|u|^{\frac{N j}{N-1}} d x\right)^{\frac{N-1}{N j}} \leq C_{4}|\Omega|^{\frac{N-1}{N j}}\left(\frac{N j}{N-1}\right)^{\frac{N-1}{N}}\|u\|_{W^{1, N}(\Omega)} .
$$

E, portanto, para cada $j \geq 1$,

$$
\begin{aligned}
\int_{\Omega}|u|^{\frac{N j}{N-1}} d x & \leq C_{4}|\Omega|\left(\frac{N j}{N-1}\right)^{j}\left(\|u\|_{W^{1, N}(\Omega)}\right)^{\frac{N j}{N-1}} \\
& =|\Omega|\left(\frac{j}{e^{\frac{N}{N-1}}}\right)^{j}\left(C_{5} e\left(\frac{N}{N-1}\right)^{\frac{N-1}{N}}\|u\|_{W^{1, N}(\Omega)}\right)^{\frac{N j}{N-1}}
\end{aligned}
$$


Note que, a serie $\sum_{j=1}^{+\infty} \frac{1}{j !}\left(\frac{j}{e^{\frac{N}{N-1}}}\right)^{j}$ é convergente pois $\frac{1}{e^{\frac{N}{N-1}}}<\frac{1}{e^{\prime}}$, logo podemos supor que

$$
\sum_{j=1}^{+\infty} \frac{1}{j !}\left(\frac{j}{e^{\frac{N}{N-1}}}\right)^{j}=C_{6}=C_{6}(N)
$$

Seja $C_{7}=\max \left\{1, C_{6}|\Omega|\right\}$ e $C_{8}=e C_{5} C_{7}\left(\frac{N}{N-1}\right)^{\frac{N-1}{N}}\|u\|_{W^{1, N}(\Omega)}=C_{9}\|u\|_{W^{1, N}(\Omega)}$, onde $C_{9}=C_{9}\left(N, \Omega, k_{\Omega}\right)$. De (1.18), temos

$$
\begin{aligned}
\int_{\Omega}\left|\frac{u}{C_{8}}\right| \frac{N j}{N-1} d x & \leq\left(\frac{1}{C_{8}}\right)^{\frac{N j}{N-1}}|\Omega|\left(\frac{j}{e^{\frac{N}{N-1}}}\right)^{j}\left(C_{5} e\left(\frac{N}{N-1}\right)^{\frac{N-1}{N}}\|u\|_{W^{1, N}(\Omega)}\right)^{\frac{N j}{N-1}} \\
& =\frac{|\Omega|\left(\frac{j}{e^{\frac{N}{N-1}}}\right)^{j}\left(C_{5} e\left(\frac{N}{N-1}\right)^{\frac{N-1}{N}}\|u\|_{W^{1, N}(\Omega)}\right)^{\frac{N j}{N-1}}}{\left(e C_{5} C_{7}\left(\frac{N}{N-1}\right)^{\frac{N-1}{N}}\|u\|_{W^{1, N}(\Omega)}\right)^{\frac{N j}{N-1}}} \\
& =|\Omega|\left(\frac{j}{e^{\frac{N}{N-1}}}\right)^{j} \frac{1}{C_{7}^{\frac{N j}{N-1}} \leq|\Omega|\left(\frac{j}{e^{\frac{N}{N-1}}}\right)^{j} \frac{1}{C_{7}}}
\end{aligned}
$$

Logo,

$$
\begin{aligned}
& \int_{\Omega} \phi\left(\frac{u}{C_{8}}\right) d x=\int_{\Omega}\left(e^{\left|\frac{u}{C_{8}}\right|^{\frac{N}{N-1}}}-1\right) d x=\int_{\Omega} \lim _{m \rightarrow \infty} \sum_{j=1}^{m} \frac{1}{j !}\left|\frac{u}{C_{8}}\right|^{\frac{N j}{N-1}} d x \\
& =\lim _{m \rightarrow \infty} \sum_{j=1}^{m} \frac{1}{j !} \int_{\Omega}\left|\frac{u}{C_{8}}\right|^{\frac{N j}{N-1}} d x \leq \lim _{m \rightarrow \infty} \sum_{j=1}^{m} \frac{1}{j !}|\Omega|\left(\frac{j}{e^{\frac{N}{N-1}}}\right)^{j} \frac{1}{C_{7}} \\
& \leq|\Omega| \frac{1}{C_{7}} \sum_{j=1}^{\infty} \frac{1}{j !}\left(\frac{j}{e^{\frac{N}{N-1}}}\right)^{j} \leq \frac{|\Omega| C_{6}}{C_{7}} \leq 1 .
\end{aligned}
$$

Portanto,

$$
\|u\|_{L^{\phi^{*}}} \leq C_{8}=C_{9}\|u\|_{W^{1, N}(\Omega)}, \quad \operatorname{com} C_{9}=C_{9}\left(N, \Omega, k_{\Omega}\right) .
$$

Pelo Lema 1.18, existe uma constante $\tilde{C}>0$, tal que $\|u\|_{W^{1, N}(\Omega)} \leq \tilde{C}\|u\|_{W^{k, p}(\Omega)}$. Consequentemente,

$$
\|u\|_{L^{\phi^{*}}} \leq C_{10}\|u\|_{W^{k, p}(\Omega)}, \quad \operatorname{com} \quad C_{10}=C_{10}\left(N, \Omega, k_{\Omega}\right)
$$

Para mostrar segunda parte do teorema, provaremos primeiro duas afirmações:

Afirmação 1: Se $\psi(t) \leq \phi(\lambda t)$ para todo $t \in \mathbb{R}, \operatorname{com} \lambda>0$. Então a seguinte imersão 
$L^{\phi^{*}}(\Omega) \subset L^{\psi^{*}}(\Omega)$ é contínua.

De fato, pela hipótese,

$$
\psi\left(\frac{u(x)}{\lambda\|u\|_{L^{\phi^{*}}}}\right) \leq \phi\left(\frac{u(x)}{\|u\|_{L^{\phi^{*}}}}\right), \text { para todo } x \in \Omega
$$

Integrando em relação a $x \in \Omega$ e do Lema 1.7

$$
\int_{\Omega} \psi\left(\frac{u(x)}{\lambda\|u\|_{L_{\phi^{*}}}}\right) d x \leq \int_{\Omega} \phi\left(\frac{u(x)}{\|u\|_{L_{\phi^{*}}}}\right) d x \leq 1
$$

Donde $\|u\|_{L^{\psi^{*}}} \leq \lambda\|u\|_{L^{\phi^{*}}}$.

Afirmação 2: Sejam $\left\{u_{n}\right\} \subset L^{\psi^{*}}(\Omega), u \in L^{\psi^{*}}(\Omega)$ tais que $\left\|u_{n}-u\right\|_{L^{\psi^{*}}}<1$, para todo $n \geq n_{0}$.

Então

$$
\int_{\Omega} \psi\left(u_{n}-u\right) d x \leq\left\|u_{n}-u\right\|_{L^{*}}, \quad \text { para todo } n \geq n_{0} .
$$

De fato, como $\phi$ é uma função convexa então $\alpha \phi(t)<\phi(\alpha t)$, para cada $\alpha>1$ logo, tomando $\alpha_{n}=\frac{1}{\left\|u_{n}-u\right\|_{L_{\phi^{*}}}}$ para cada $n \geq n_{0}$, temos

$$
\int_{\Omega} \frac{\phi\left(u_{n}-u\right)}{\left\|u_{n}-u\right\|_{L^{\phi^{*}}}} d x \leq \int_{\Omega} \phi\left(\frac{u_{n}-u}{\left\|u_{n}-u\right\|_{L^{\phi^{*}}}}\right) d x \leq 1 .
$$

Donde segue a afirmação.

Agora provemos a segunda parte do teorema. Seja $u \in W^{k, p}(\Omega)$, então existe $\left\{u_{n}\right\} \subset$ $L^{\infty}(\Omega)$, tal que $\left\|u_{n}-u\right\|_{W^{k, p}} \rightarrow 0$, pela imersão do Teorema 1.19 , tem-se $\left\|u_{n}-u\right\|_{L^{\phi^{*}}} \rightarrow 0$ portanto, $u \in E^{\phi}(\Omega)=\overline{L^{\infty}}(\Omega)^{\|\cdot\|_{L^{\phi^{*}}}}$. Pelo Lema 1.11, $u \in L^{\phi}(\Omega) \subset L^{\phi^{*}}(\Omega)$. Pela Afirmação $1, u \in L^{\psi^{*}}$ e $\left\|u_{n}-u\right\|_{L^{\psi^{*}}} \rightarrow 0$ e finalmente pela Afirmação 2, temos

$$
\int_{\Omega} \psi\left(u_{n}-u\right) d x \rightarrow 0, \quad \text { se } n \rightarrow \infty
$$

E, portanto, $W^{k, p}(\Omega)$ está imerso continuamente no sentido na convergência $L^{\psi}(\Omega)$, em qualquer classe de Orlicz $L^{\psi}(\Omega)$, onde $\psi(t) \leq \phi(\lambda t)$, com $\lambda>0$.

\subsection{Otimalidade das imersões nos espaços de Orlicz}

As imersões citadas nos Teoremas 1.16 e 1.19 são ainda válidas se consideramos os espaços $W_{0}^{1,1,1}(\Omega)$ e $W_{0}^{k, p}(\Omega), N=k p$ no lugar dos espaços $W^{1,1,1}(\Omega)$ e $W^{k, p}(\Omega), N=k p$ respectivamente, sem precisar supor que e $\Omega$ seja limitado e satisfaça a condição 
do cone . Para ver isto, é suficiente usar o Lema 1.14 no lugar do Lema 1.13 nas demonstrações.

Mostremos que as imersão dos teoremas são ótimas. Consideramos a seguinte função $u: B_{1}(0) \rightarrow \mathbb{R}$ definida como $u(x)=\ln \frac{1}{|x|}$. Logo,

$$
\int_{B_{1}(0)} \ln \frac{1}{|x|} d x=-\omega_{N} \int_{0}^{1} r^{N-1} \ln r d x=\frac{\omega_{N}}{N^{2}}
$$

e

$$
\int_{B_{1}(0)} \frac{1}{|x|} d x=\omega_{N} \int_{0}^{1} r^{N-2} d r=\frac{\omega_{N}}{N-1}
$$

assim $u \in W^{1,1}\left(B_{1}(0)\right)$. Por outro lado, seja $B_{R}\left(x_{0}\right) \subset \mathbb{R}^{N}$ uma bola de raio $R$

$$
\begin{aligned}
\int_{B_{1}(0) \cap B_{R}\left(x_{0}\right)}|\nabla u| d x & =\int_{B_{1}(0) \cap B_{R}\left(x_{0}\right)} \frac{1}{|x|} d x \leq \int_{B_{R}\left(x_{0}\right)} \frac{1}{|x|} d x \\
& \leq \int_{B_{R}(0)} \frac{1}{|x|} d x=\omega_{N} \int_{0}^{R} R^{N-2} d r=\frac{\omega_{N} R^{N-1}}{N-1} \\
& =\frac{\omega_{N}^{\frac{1}{N}}}{N-1}\left(\omega_{N} R^{N}\right)^{1-\frac{1}{N}}=\frac{\omega_{N}^{\frac{1}{N}}}{N-1}\left|B_{R}\left(x_{0}\right)\right|^{1-\frac{1}{N}} .
\end{aligned}
$$

Portanto, $\nabla u \in L^{1,1}\left(B_{1}(0)\right)$, donde $u \in W^{1,1,1}\left(B_{1}(0)\right)$. Porém $u \notin L^{\phi}\left(B_{1}(0)\right)$, para a N-função $\phi(t)=e^{b|t|}-b|t|-1, \operatorname{com} b>N$, pois

$$
\int_{B_{1}(0)} e^{b \ln \frac{1}{|x|}} d x=\omega_{N} \int_{0}^{1} r^{N-1-b} d r=\lim _{r \rightarrow 0^{+}} \frac{r^{N-b}}{N-b}=+\infty
$$

como $\int_{B_{1}(0)} \ln \frac{1}{|x|} d x=\frac{\omega_{N}}{N^{2}} \mathrm{e} \int_{B_{1}(0)} 1 d x=\omega_{N}$, temos

$$
\int_{B_{1}(0)}\left(e^{b \ln \frac{1}{|x|}}-b \ln \frac{1}{|x|}-1\right) d x=+\infty
$$

Isto mostra que $W^{1,1,1}(\Omega)$, não pode ser imerso em $L^{\phi}(\Omega)$, onde $\phi(t)=e^{b|t|}-b|t|-1$, $b>N$. Consequentemente, é necessário considerar o espaço $L^{\phi^{*}}(\Omega)$ no Teorema 1.16 .

Por outro lado, a constante $N /(N-1)$ da imersão do Teorema 1.19, é ótima, isto é que o espaço $W^{1, N}(\Omega)$, não pode ser imerso no espaço de Orlicz $L^{\phi^{*}}(\Omega)$, onde

$$
\phi(t)=e^{|t|^{\gamma}}-1, \quad \text { e } \quad \gamma>\frac{N}{N-1} .
$$


Para mostrar isto, consideremos a seguinte função radial $u$ definida em $B_{1}(0) \subset \mathbb{R}^{N}$

$$
u(x)=\left(\ln \frac{1}{|x|}\right)^{\gamma^{\prime}}, \quad \text { onde } \frac{N-1}{N}>\gamma^{\prime}>\frac{1}{\gamma} .
$$

Logo, para cada $k>0$ e cada $n \geq 1$, temos

$$
\int_{B_{1}(0)} e^{\frac{1}{k \gamma}\left(\ln \frac{1}{|x|}\right)^{\gamma^{\prime} \gamma}} d x=\omega_{N} \int_{0}^{1} r^{N-1} e^{\frac{1}{k \gamma}\left(\ln \frac{1}{r}\right)^{\gamma^{\prime} \gamma}} d r \geq \omega_{N} \int_{0}^{\frac{1}{n}} r^{N-1} e^{\frac{1}{k \gamma}\left(\ln \frac{1}{r} \gamma^{\gamma^{\prime} \gamma}\right.} d r .
$$

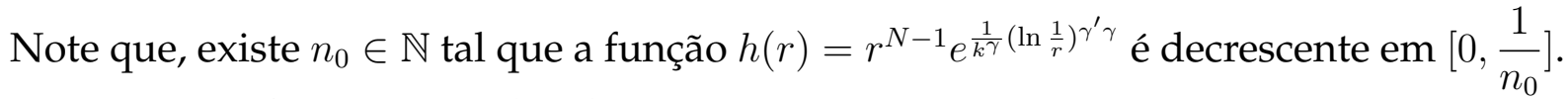
Logo, para cada $n \geq n_{0}$ em (1.20), temos

$$
\int_{B_{1}(0)} e^{\frac{1}{k^{\gamma}}\left(\ln \frac{1}{|x|}\right)^{\gamma^{\prime} \gamma}} d x \geq \omega_{N} \int_{0}^{\frac{1}{n}} r^{N-1} e^{\frac{1}{k^{\gamma}}\left(\ln \frac{1}{r}\right)^{\gamma^{\prime} \gamma}} d r \geq \omega_{N} \frac{e^{\frac{1}{k^{\gamma}}(\ln n)^{\gamma^{\prime} \gamma}}}{n^{N-1}} \frac{1}{n}
$$

Como $\gamma^{\prime} \gamma>1$, tem-se $\lim _{n \rightarrow+\infty} \frac{e^{\frac{1}{k^{\gamma}}(\ln n)^{\gamma^{\prime} \gamma}}}{n^{N-2}}=+\infty$, portanto $\int_{B_{1}(0)} e^{\frac{1}{k \gamma\left(\ln \frac{1}{|x|}\right)^{\gamma^{\prime} \gamma}}} d x=+\infty$. Deste modo, para cada $k>0$

$$
\int_{B_{1}(0)} \phi\left(\frac{u(x)}{k}\right) d x=\int_{B_{1}(0)}\left(e^{\frac{1}{k \gamma}\left(\ln \frac{1}{|x|}\right)^{\gamma^{\prime} \gamma}}-1\right) d x=+\infty
$$

$\operatorname{assim} u \notin L^{\phi^{*}}\left(B_{1}(0)\right)$, mas $u \in W^{1, N}(\Omega)$, pois

$$
\int_{B_{1}(0)}|\nabla u|^{N} d x=\omega_{N} \int_{0}^{1}\left|\left(\ln \frac{1}{r}\right)^{\gamma^{\prime}-1} \frac{1}{r}\right|^{N} r^{N-1} d r=\omega_{N} \int_{0}^{1} \frac{d r}{r\left(\ln \frac{1}{r}\right)^{-N\left(\gamma^{\prime}-1\right)}}<+\infty
$$

onde a última integral é finita, pois $-N\left(\gamma^{\prime}-1\right)>1$. 


\section{Capítulo}

\section{A desigualdade de Trudinger-Moser}

O principal objetivo deste capítulo é provar uma desigualdade chamada de Trudinger-Moser em um domínio limitado $\Omega \subset \mathbb{R}^{N}$, que estabelece a existência de uma constante $C=C(N)>0$ tal que para $\alpha \leq \alpha_{N}$.

$$
\int_{\Omega} e^{\alpha u^{\frac{N}{N-1}}} d x \leq C|\Omega|, \quad \forall u \in W_{0}^{1, N}(\Omega), \operatorname{com}\|\nabla u\|_{L^{N}} \leq 1
$$

onde $\omega_{N-1}$ é a medida da esfera unitária $(N-1)$-dimensional e $\alpha_{N}=N \omega_{N-1}^{\frac{1}{N}-1}$. Além disso, a constante $\alpha_{N}$ é ótima isto é, se $\alpha>\alpha_{N}$, a integral acima pode ser arbitrariamente grande, para alguma função apropriada $u$. A prova deste resultado faz uso da simetrização de Schwarz, o qual reduz o problema N-dimensional a um problema unidimensional. Este capítulo é baseada no artigo [11] de Moser.

\subsection{Simetrização de Schwarz}

Definição 2.1. Sejam $\Omega \subset \mathbb{R}^{N}$ um conjunto limitado e $\varphi: \Omega \rightarrow \mathbb{R}$ uma função simples positiva, isto é

$$
\varphi=\sum_{j=1}^{n} a_{j} \chi_{A_{j}},
$$

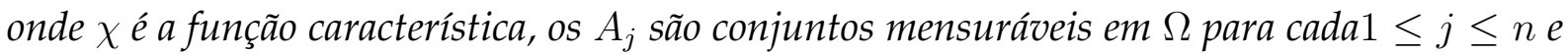
$0 \leq a_{n}<a_{n-1}<, \ldots,<a_{1}$. 
Definimos $\varphi^{*}: \Omega^{*} \rightarrow \mathbb{R}$, a simetrização de Schwarz de $\varphi$ como

$$
\varphi^{*}=\sum_{j=1}^{n} a_{j} \chi_{\left\{R_{j-1} \leq|x|<R_{j}\right\}}
$$

onde $\Omega^{*}=B_{R}(0)$, com $R>0$ tal que $\left|B_{R}(0)\right|=|\Omega|, R_{0}=0$ e os $R_{j}$ são números positivos tais que satisfazem a condição $\left|\left\{R_{j-1} \leq|x|<R_{j}\right\}\right|=\left|A_{j}\right|$, para cada $1 \leq j \leq n$.

Exemplo 2.2. Seja a função simples $\varphi:[2,16] \rightarrow \mathbb{R}$, definida por

$$
\varphi(x)= \begin{cases}2, & 2 \leq x<4 \\ 9, & 4 \leq x<8 \\ 4, & 8 \leq x<14 \\ 5, & 14 \leq x \leq 16\end{cases}
$$

Então, a simetrização de Schwarz de $\varphi$ é dado por $\varphi^{*}:(-7,7) \mapsto \mathbb{R}$, onde

$$
\varphi^{*}(x)= \begin{cases}9, & 0 \leq|x|<2, \\ 5, & 2 \leq|x|<3 \\ 4, & 3 \leq|x|<6 \\ 2, & 6 \leq|x|<7\end{cases}
$$

Veja as figuras

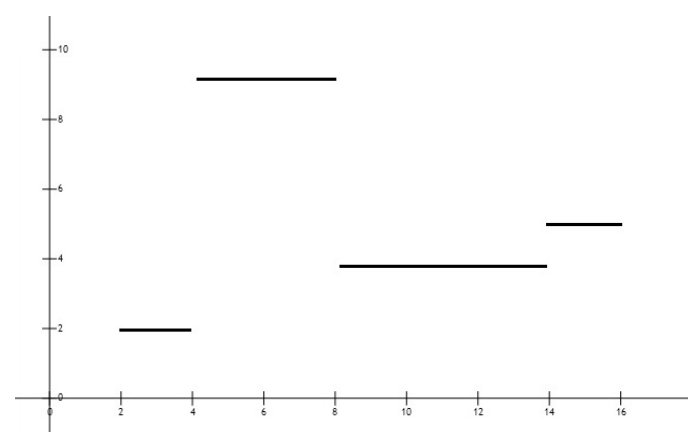

Figura 2.1: função $\varphi$

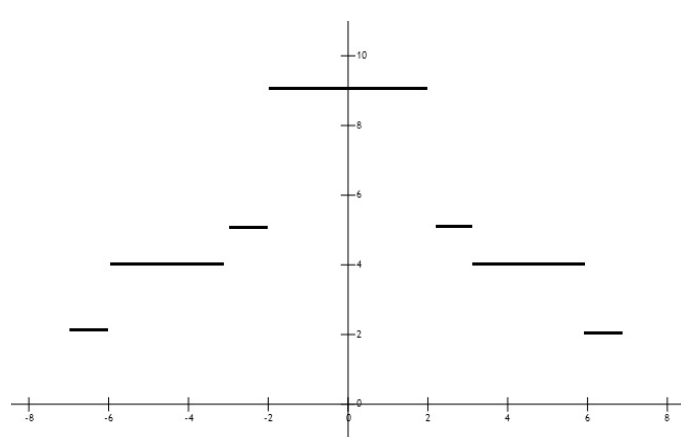

Figura 2.2: função $\varphi^{*}$

Observação 2.3. A simetrização de Schwarz $\varphi^{*}$ satisfaz as seguintes propriedades:

(i) $\varphi^{*}$ é uma função positiva, radialmente simétrica (i.e. $\varphi^{*}(x)=\varphi^{*}(y)$, se $|x|=|y|$.) e radialmente não crescente (i.e. $\varphi^{*}(x) \leq \varphi^{*}(y)$, se $|y| \leq|x|$ ).

(ii) Se $0 \leq \varphi_{1} \leq \varphi_{2}$, então $0 \leq \varphi_{1}^{*} \leq \varphi_{2}^{*}$. 
Definição 2.4. Seja $f$ uma função positiva. Defina $f^{*}$ a simetrização de Schwarz de $f$ como $f^{*}=\lim _{n \rightarrow \infty} f_{n}^{*}$, onde $\left\{f_{n}\right\}$ é uma sequência de funções simples, positivas e crescentes tal que $f=\lim _{n \rightarrow \infty} f_{n}$.

Note que se $f$ é uma função positiva, então $f^{*}$ satisfaz também as propriedades da Observação 2.3.

Proposição 2.5. Seja $f: \Omega \rightarrow \mathbb{R}$ uma função positiva. Então

a) Seja $G: \mathbb{R} \rightarrow \mathbb{R}$, uma função contínua, positiva é crescente, tal que $G(f) \in L^{1}(\Omega)$, então $G\left(f^{*}\right) \in L^{1}(\Omega) e$

$$
\int_{\Omega} G(f(x)) d x=\int_{\Omega^{*}} G\left(f^{*}(x)\right) d x
$$

b) A seguinte desigualdade é valida (Desigualdade de Pólya-Szegö):

$$
\left.\int_{\Omega^{*}}\left|\nabla f^{*}(x)\right|^{N} d x \leq \int_{\Omega} \mid \nabla f^{(} x\right)\left.\right|^{N} d x
$$

\section{Demonstração.}

a) Seja $\varphi=\sum_{j=1}^{n} a_{j} \chi_{A_{j}}: \Omega \rightarrow \mathbb{R}$ uma função simples, então

$$
\begin{aligned}
\int_{\Omega} \varphi(x) d x & =\sum_{j=1}^{n} a_{j}\left|A_{j}\right|=\sum_{j=1}^{n} a_{j}\left|\chi_{\left\{R_{j-1} \leq|x|<R_{j}\right\}}\right| \\
& =\sum_{j=1}^{n} a_{j} \int_{B_{R}(0)} \chi_{\left\{R_{j-1} \leq|x|<R_{j}\right\}} d x=\int_{\Omega^{*}} \varphi^{*}(x) d x .
\end{aligned}
$$

Seja $f=\lim _{n \rightarrow \infty} f_{n}$, onde $\left\{f_{n}\right\}$ uma sequência de funções simples positivas e crescentes. Pela Observação 2.3, $\left\{f_{n}^{*}\right\}$ é uma sequência de funções simples positivas e crescentes com $\lim _{n \rightarrow \infty} f_{n}^{*}=f^{*}$. Logo, como $G$ é contínua positiva e crescentes, temos $\left\{G\left(f_{n}\right)\right\}$ e $\left\{G\left(f_{n}^{*}\right)\right\}$ são sequencias simples positivas e crescentes com

$$
G(f)=\lim _{n \rightarrow \infty} G\left(f_{n}\right) \quad \text { e } \quad G\left(f^{*}\right)=\lim _{n \rightarrow \infty} G\left(f_{n}^{*}\right) .
$$

Pelo Teorema da Convergência Monótona e de (2.1), temos

$$
\int_{\Omega} G(f(x)) d x=\lim _{n \rightarrow \infty} \int_{\Omega} G\left(f_{n}(x)\right) d x=\lim _{n \rightarrow \infty} \int_{\Omega^{*}} G\left(f_{n}^{*}(x)\right) d x=\int_{\Omega^{*}} G\left(f^{*}(x)\right) d x .
$$


b) Uma prova de $b$ ) pode ser encontrada em [12].

\subsection{Lemas}

Agora vamos a provar os lemas necessários para a demonstração da desigualdade de Trundiger-Moser.

Lema 2.6. Seja $y:[0,+\infty) \rightarrow \mathbb{R}$, uma função satisfazendo

$$
y(0)=0, \int_{0}^{\infty} y^{\prime}(s)^{N} d s \leq 1, y^{\prime}(s) \geq 0 \text { para } s \geq 0 e \frac{y(s)^{N}}{s^{N-1}} \leq 1-\delta=y_{1}^{N} .
$$

Então

$$
(N-1) y_{1}^{N-2} \int_{0}^{1}\left(y^{\prime}(s)-y_{1}\right)^{2} d s+\int_{0}^{\infty} y^{\prime}(s)^{N} d s \leq \delta
$$

onde $y_{1}:=y(1)$.

Demonstração. Pela desigualdade $(i)$ do Lema B.3, para cada $s \geq 0$, com $a=y_{1}$ e $b=y^{\prime}(s)-y_{1}$, temos

$$
y_{1}^{N}+N y_{1}^{N-1}\left(y^{\prime}(s)-y_{1}\right)+(N-1) y_{1}^{N-2}\left(y^{\prime}-y_{1}\right)^{2} \leq y^{\prime}(s)^{N}, \quad \text { se } s \geq 0 .
$$

Integrando na variável $s$ em $[0,1]$.

$$
y_{1}^{N}+N y_{1}^{N-1} \int_{0}^{1}\left(y^{\prime}(s)-y_{1}\right) d s+(N-1) y_{1}^{N-2} \int_{0}^{1}\left(y^{\prime}-y_{1}\right)^{2} d s \leq \int_{0}^{1} y^{\prime}(s)^{N} d s .
$$

Note que,

$$
\int_{0}^{1}\left(y^{\prime}(s)-y_{1}\right) d s=y(1)-y(0)-y_{1}=0
$$

$\mathrm{e}$

$$
\int_{0}^{1} y^{\prime}(s)^{N} d s=\int_{0}^{\infty} y^{\prime}(s)^{N} d s-\int_{1}^{\infty} y^{\prime}(s)^{N} d s \leq 1-\int_{1}^{\infty} y^{\prime}(s)^{N} d s=\delta+y_{1}^{N}-\int_{1}^{\infty} y^{\prime}(s)^{N} d s .
$$

Logo, substituindo em (2.3), temos

$$
y_{1}^{N}+(N-1) y_{1}^{N-2} \int_{0}^{1}\left(y^{\prime}-y_{1}\right)^{2} d s \leq \int_{0}^{1} y^{\prime}(s)^{N} d s \leq \delta+y_{1}^{N}-\int_{1}^{\infty} y^{\prime}(s)^{N} d s .
$$

Donde concluímos a demonstração do lema. 
Lema 2.7. Seja $y:[0,+\infty) \rightarrow \mathbb{R}$ uma função satisfazendo (2.2) e $\frac{1}{p}+\frac{1}{N}=1$. Então para cada $0<\delta<\frac{1}{2}$, temos $y(s) \leq z(s)$, para todo $s \geq 0$, onde

$$
z(s)=\left\{\begin{array}{ll}
s+\min \left\{(2 \delta)^{\frac{1}{N}} s^{\frac{1}{p}}, C_{3}(\delta(1-\delta))^{\frac{1}{2}}\right\}, & 0 \leq s \leq 1, \\
1+\delta \frac{1}{N}(s-1)^{\frac{1}{p}}, & 1<s
\end{array} \quad C_{3}=C_{3}(N) .\right.
$$

Demonstração. De (2.2), temos

$$
\int_{0}^{\infty} y^{\prime}(s)^{N} d s \leq \delta, \quad \int_{0}^{1}\left(y^{\prime}(s)-y_{1}\right)^{2} d s \leq \frac{\delta}{y_{1}^{N-2}(N-1)} .
$$

- Se $s>1$

$$
\begin{aligned}
y(s)-y(1) & =\int_{1}^{s} y^{\prime}(w) d w \leq\|1\|_{L^{p}([1, s])}\left\|y^{\prime}\right\|_{L^{N}([1, s])}=\left(\int_{1}^{s} d w\right)^{\frac{1}{p}}\left(\int_{1}^{s} y^{\prime}(w)^{N} d w\right)^{\frac{1}{N}} \\
& \leq(s-1)^{\frac{1}{p}}\left(\int_{1}^{\infty} y^{\prime}(s)^{N} d s\right)^{\frac{1}{N}} \leq(s-1)^{\frac{1}{p}} \delta^{\frac{1}{N}} .
\end{aligned}
$$

E, portanto,

$$
y(s) \leq 1+(s-1)^{\frac{1}{p}} \delta^{\frac{1}{N}}, \quad \text { se } s>1 .
$$

- Se $0 \leq s \leq 1$

Note que

$$
\int_{s}^{1}\left(y_{1}-y^{\prime}(w)\right) d w=y_{1}-y(1)-y_{1} s+y(s)=y(s)-y_{1} s .
$$

E, por conseguinte, usando a desigualdade de Schwarz e (2.5), temos

$$
\begin{aligned}
y(s)-y_{1} s & =\int_{s}^{1} y_{1}-y^{\prime}(w) d w \leq \int_{s}^{1}\left|y^{\prime}(w)-y_{1} s\right| d w \\
& \leq\left(\int_{s}^{1} d w\right)^{\frac{1}{2}}\left(\int_{s}^{1}\left(y^{\prime}(w)-y_{1}\right)^{2} d w\right)^{\frac{1}{2}} \leq(1-s)^{\frac{1}{2}}\left(\frac{\delta}{y_{1}^{N-2}(N-1)}\right)^{\frac{1}{2}} \\
& =C_{3}(N)(\delta(1-s))^{\frac{1}{2}} .
\end{aligned}
$$

Como $y_{1} \leq 1$, temos

$$
y(s) \leq s+C_{3}(\delta(1-s))^{\frac{1}{2}}, \text { se } 0 \leq s \leq 1 .
$$

Seja $0<\sigma<1$ fixado, achemos o máximo de $y(\sigma)$, para todas as funções $y$ 
satisfazendo (2.7) e as seguinte condições

$$
y(0)=0, \quad y(1)=y_{1}, \quad \int_{0}^{1} y^{\prime}(s)^{N} d s \leq 1
$$

Seja $y^{*}$ tal máximo que é atingido por uma função que é um segmento de reta passando por $(0,0)$ e $\left(\sigma, y^{*}\right)$ junto com outro segmento de reta unindo $\left(\sigma, y^{*}\right)$ com $\left(1, y_{1}\right)$. Da condição $\int_{0}^{1} y^{\prime}(s)^{N} d s \leq 1$, temos

$$
\int_{0}^{1} y^{\prime}(s)^{N} d s=\int_{0}^{\sigma}\left(\frac{y^{*}}{\sigma}\right)^{N} d s+\int_{\sigma}^{1}\left|\frac{y_{1}-y^{*}}{1-\sigma}\right|^{N} d s \leq 1 .
$$

E, portanto,

$$
\left(\frac{y^{*}}{\sigma}\right)^{N} \sigma+\left|\frac{y_{1}-y^{*}}{1-\sigma}\right|^{N}(1-\sigma) \leq 1
$$

Como $\frac{y^{*}}{\sigma} \geq y_{1}$, então $y^{*}=y_{1}(\sigma+\rho) \operatorname{com} \rho \geq 0$ logo, substituindo na equação anterior

$$
y_{1}^{N}\left(1+\frac{\rho}{\sigma}\right)^{N} \sigma+y_{1}^{N}\left|1-\frac{\rho}{1-\sigma}\right|^{N}(1-\sigma) \leq 1
$$

E, portanto,

$$
\left(1+\frac{\rho}{\sigma}\right)^{N} \sigma+\left|1-\frac{\rho}{1-\sigma}\right|^{N}(1-\sigma) \leq \frac{1}{y_{1}^{N}}=\frac{1}{1-\delta} .
$$

Pela desigualdade (i) do Lema B.3, temos $1+N \frac{\rho}{\sigma}+\left(\frac{\rho}{\sigma}\right)^{N} \leq\left(1+\frac{\rho}{\sigma}\right)^{N}$.

Observe que como $1-N x \leq|1-x|^{N}$ para cada $x \in \mathbb{R}$, temos

$$
1-N \frac{\rho}{1-\sigma} \leq\left|1-\frac{\rho}{1-\sigma}\right|^{N}
$$

Substituindo as últimas desigualdades em (2.9), temos

$$
\left(1+N \frac{\rho}{\sigma}+\left(\frac{\rho}{\sigma}\right)^{N}\right) \sigma+\left(1-N \frac{\rho}{1-\sigma}\right)(1-\sigma) \leq \frac{1}{1-\delta}
$$

Simplificando

$$
\frac{\rho^{N}}{\sigma^{N-1}}+1 \leq \frac{1}{1-\delta}, \quad \text { ou seja, } \frac{\rho^{N}}{\sigma^{N-1}} \leq \frac{\delta}{1-\delta}
$$

Note que $\frac{1}{1-\delta}<2$, pois $0<\delta<\frac{1}{2}$ e, por conseguinte,

$$
\rho^{N} \leq 2 \delta \sigma^{N-1} \quad \text { e, portanto, } \quad \rho \leq(2 \delta)^{\frac{1}{N}} \sigma^{\frac{1}{p}}
$$


Então, para cada $y$ satisfazendo (2.8), temos

$$
y(\sigma) \leq y^{*}=y_{1}(\sigma+\rho) \leq \sigma+\rho \leq \sigma+(2 \delta)^{\frac{1}{N}} \sigma^{\frac{1}{p}}
$$

Como $\sigma$ foi arbitrário em $(0,1)$

$$
y(s) \leq s+(2 \delta)^{\frac{1}{N}} s^{\frac{1}{p}}, \quad \text { se } 0 \leq s \leq 1 .
$$

Portanto, de (2.7) e (2.10), resulta

$$
y(s) \leq \min \left\{C_{3}(\delta(1-s))^{\frac{1}{2}}, s+(2 \delta)^{\frac{1}{N}} s^{\frac{1}{p}}\right\}, \quad \text { se } 0 \leq s \leq 1
$$

Lema 2.8. Seja $z:[0,+\infty) \rightarrow \mathbb{R}$ a função definida em (2.4).

Defina a função :

$$
\varphi(s)= \begin{cases}s, & 0 \leq s \leq \frac{1}{2} \\ |s-1|, & \frac{1}{2}<s .\end{cases}
$$

Então existe $0<\delta_{0}<\frac{1}{2}$ e constantes $C_{4}=C_{4}(N) ; C_{5}=C_{5}(N)$, tal que para cada $0<\delta<\delta_{0}$

$$
z(s)^{p}-s \leq-\frac{\varphi(s)}{C_{5}}, \quad \text { se } s \notin \Delta:=\left\{s \in \mathbb{R}^{+}:|s-1|<C_{4} \delta\right\}
$$

Demonstração. Seja $0<s<\frac{1}{2}$, pela segunda parte da desigualdade (ii) do Lema B.3

$$
\begin{aligned}
z(s)^{p} & \leq\left(s+(2 \delta)^{\frac{1}{N} s^{\frac{1}{p}}}\right)^{p} \leq s^{p}+C_{7}\left(s^{p-1}(2 \delta)^{\frac{1}{N}} s^{\frac{1}{p}}+(2 \delta)^{\frac{p}{N}} s\right) \\
& =s^{p}+C_{7}\left(s^{p+\frac{1}{p}-1}(2 \delta)^{\frac{1}{N}}+s(2 \delta)^{\frac{1}{N-1}}\right) .
\end{aligned}
$$

Como $s^{p+\frac{1}{p}-1} \leq s$ e $(2 \delta)^{\frac{1}{N-1}}<(2 \delta)^{\frac{1}{N}}$, temos

$$
z(s)^{p}-s \leq s^{p}-s+2 C_{7} s(2 \delta)^{\frac{1}{N}}=s\left(s^{p-1}-1+2 C_{7}(2 \delta)^{\frac{1}{N}}\right)
$$

Além disso, como $s^{p-1}<\left(\frac{1}{2}\right)^{p-1}<\left(\frac{1}{2}\right)^{\frac{p-1}{2}}$. Então existe $\delta_{0}>0$ suficientemente pequeno tal que para $0<\delta<\delta_{0}$, tem-se $s^{p-1}+2 C_{7}(2 \delta)^{\frac{1}{2}}<\left(\frac{1}{2}\right)^{\frac{p-1}{2}}$ e, portanto,

$$
z(s)^{p}-s \leq s\left(\left(\frac{1}{2}\right)^{\frac{p-1}{2}}-1\right)=-\varphi(s)\left(1-\left(\frac{1}{2}\right)^{\frac{p-1}{2}}\right), \quad \text { se } 0 \leq s<\frac{1}{2}
$$


Para $\frac{1}{2} \leq s<1$, considere $\sigma=1-s$, logo de (2.4) e a segunda desigualdade de (ii) do Lema B.3

$$
z(s)^{p}-s \leq\left(s+C_{3}(\delta \sigma)^{\frac{1}{2}}\right)^{p}-s \leq s^{p}+C_{7} C_{3} s^{p-1}(\delta \sigma)^{\frac{1}{2}}+C_{7}\left(C_{3}(\delta \sigma)^{\frac{1}{2}}\right)^{p}-s
$$

Como $s^{p-1}<1$ e $(\delta \sigma)^{\frac{p}{2}}<(\delta \sigma)^{\frac{1}{2}}$, então

$$
\begin{aligned}
z(s)^{p}-s & \leq s\left(s^{p-1}-1\right)+C_{7} C_{3}(\delta \sigma)^{\frac{1}{2}}+C_{7} C_{3}^{p}(\delta \sigma)^{\frac{1}{2}}=s\left(s^{p-1}-1\right)+C_{8}(\delta \sigma)^{\frac{1}{2}} \\
& =s\left((1-\sigma)^{p-1}-1\right)+C_{8}(\delta \sigma)^{\frac{1}{2}} \leq-s(p-1) \sigma+C_{8}(\delta \sigma)^{\frac{1}{2}} \\
& \leq-\frac{(p-1) \sigma}{2}+C_{8}(\delta \sigma)^{\frac{1}{2}} .
\end{aligned}
$$

Agora, para $\left(\frac{4 C_{8}}{p-1}\right)^{2} \delta<1-s=\sigma$, temos $C_{8}<\left(\frac{\sigma}{\delta}\right)^{\frac{1}{2}} \frac{(p-1)}{4} ; \operatorname{logo}$

$$
\begin{aligned}
z(s)^{p}-s & \leq-\frac{(p-1) \sigma}{2}+C_{8}(\delta \sigma)^{\frac{1}{2}} \leq-\frac{(p-1) \sigma}{2}+\left(\frac{\sigma}{\delta}\right)^{\frac{1}{2}} \frac{(p-1)}{4}(\delta \sigma)^{\frac{1}{2}} \\
& =-\frac{(p-1) \sigma}{4}=-\frac{(p-1)(1-s)}{2} .
\end{aligned}
$$

Por conseguinte,

$$
z(s)^{p}-s \leq-\frac{(p-1)}{4} \varphi(s) \text { se } \quad\left(\frac{4 C_{8}}{p-1}\right)^{2} \delta<1-s .
$$

Para $s>1$, de (2.4) e segunda parte da desigualdade (ii) do Lema B.3

$$
\begin{aligned}
z(s)^{p}-s & \leq\left(1+\delta^{\frac{1}{N}}(s-1)^{\frac{1}{p}}\right)^{p}-s \leq 1+C_{7}\left(\delta^{\frac{1}{N}}(s-1)^{\frac{1}{p}}+C_{7} \delta^{\frac{p}{N}}(s-1)\right)-s \\
& =(s-1)\left(-1+C_{7}\left(\frac{\delta}{s-1}\right)^{\frac{1}{N}}+C_{7} \delta^{\frac{p}{N}}\right) .
\end{aligned}
$$

Considerando $\delta_{0}>0$ suficientemente pequeno tal que $C_{7} \delta_{0}^{p / N}<\frac{1}{2}$. Logo, se $0<\delta<\delta_{0}$, temos

$$
z(s)^{p}-s \leq(s-1)\left(-\frac{1}{2}+C_{7}\left(\frac{\delta}{s-1}\right)^{\frac{1}{N}}\right) .
$$

Além disso, para $(s-1)>\left(4 C_{7}\right)^{N} \delta$, temos $C_{7}\left(\frac{\delta}{s-1}\right)^{\frac{1}{N}}<\frac{1}{4}$.

Portanto, para $0<\delta<\delta_{0}$, resulta

$$
z(s)^{p}-s \leq(s-1)\left(-1+\frac{1}{4}\right) \leq-\frac{1}{4}(s-1) .
$$


Consequentemente,

$$
z(s)^{p}-s \leq-\frac{1}{4} \varphi(s) \quad \text { se } \quad\left(4 C_{7}\right)^{N} \delta<s-1
$$

Tomando

$$
C_{4}=\left(\frac{\left.4 C_{8}\right)}{p-1}\right)^{2} \delta+\left(4 C_{7}\right)^{N}, \quad C_{5}=\left(1-2^{\frac{1-p}{p}}\right)^{-1}+\frac{4}{p-1}, \quad \delta_{0}<\min \left\{\left(\frac{1}{2 C_{7}}\right)^{\frac{N}{p}}, \frac{1}{2 C_{4}}\right\} .
$$

Logo, usando (2.13), (2.14) e (2.15) para $0<\delta<\delta_{0}$, temos

$$
z(s)^{p}-s \leq-\frac{\varphi(s)}{C_{5}}, \quad \text { se } s \notin \Delta:=\left\{s \in \mathbb{R}^{+}:|s-1|<C_{4} \delta\right\}
$$

\subsection{A desigualdade de Trudinger-Moser}

Teorema 2.9. Sejam $\Omega \subset \mathbb{R}^{N}, N \geq 2$, um domínio limitado e $u \in W_{0}^{1, N}(\Omega)$ tal que

$$
\int_{\Omega}|\nabla u(x)|^{N} d x \leq 1
$$

Então existe uma constante $C=C(N)$, tal que

$$
\int_{\Omega} e^{\alpha u^{p}} d x \leq C|\Omega|
$$

onde

$$
p=\frac{N}{N-1}, \quad \alpha \leq \alpha_{N}=N \omega_{N-1}^{\frac{1}{N}-1}
$$

e $\omega_{N-1}$ é a medida da esfera unitária $(N-1)$-dimensional. Além disso, se $\alpha>\alpha_{N}$, a integral em (2.17) pode ser arbitrariamente grande, para uma função apropriada $u$.

Demonstração. Note que basta provar para $u \geq 0$, pois como $|\nabla u|=\nabla|u|$, podemos substituir $u$ por $|u|$ sem alterar (2.16), e sendo válido (2.17) para a função $|u|$ também seria válido para a função $u$.

Seja $u^{*}$ a simetrização de Schwarz de $u$, usando a Proposição 2.5 para a função $G(t)=e^{\alpha t^{p}}$ temos

$$
\int_{\Omega} e^{\alpha u^{p}} d x=\int_{\Omega^{*}} e^{\alpha u^{* p}} d x
$$

Dado que $u^{*}$ é uma função radial podemos escrever $u^{*}(x)=v(r)$, onde $r=|x|$. Defina 
a função

$$
\begin{aligned}
w:[0,+\infty) & \rightarrow \mathbb{R} \\
t & \mapsto w(t)=n^{N-\frac{1}{N}} \omega_{N}^{\frac{1}{N}} v(r), \quad r=R e^{-\frac{t}{N}}
\end{aligned}
$$

Como $v$ é função não crescente, então $w$ é uma função não decrescente. Além disso,

(i) $\int_{\Omega^{*}}\left|\nabla u^{*}(x)\right| d x=\int_{0}^{\infty} w^{\prime}(t)^{N} d t$

(ii) $\frac{1}{|\Omega|} \int_{\Omega^{*}} e^{\alpha u^{* p}} d x=\int_{0}^{\infty} e^{\beta w^{p}-t} d t$, onde $\beta=\frac{\alpha}{\alpha_{N}}$.

De fato, verificaremos $(i)$ primeiramente. Como $u^{*}(x)=v(|x|)$, então $\nabla u^{*}(x)=$ $v^{\prime}(|x|) \frac{x}{|x|}, \log \mathrm{o},\left|\nabla u^{*}(x)\right|^{N}=\left|v^{\prime}(r)\right|^{N}$. Além disso,

$$
w^{\prime}(t)=n^{N-\frac{1}{N}} \omega_{N}^{\frac{1}{N}} v^{\prime}(r) \frac{d r}{d t}, \quad d r=-\frac{r}{N} d t, \quad\left|v^{\prime}(r)\right|^{N}=\frac{N}{r^{N} \omega_{N-1}} w^{\prime}(t)^{N} .
$$

Fazendo a mudança de variáveis e usando coordenadas radiais

$$
\begin{aligned}
\int_{\Omega^{*}}\left|\nabla u^{*}(x)\right|^{N} d x & =\int_{B_{R}(0)}\left|\nabla u^{*}(x)\right|^{N} d x=\omega_{N-1} \int_{0}^{R}\left|v^{\prime}(r)\right|^{N} r^{N-1} d r \\
& =\omega_{N-1} \int_{\infty}^{0} \frac{N}{r^{N} \omega_{N-1}} w^{\prime}(t)^{N} r^{N-1}\left(-\frac{r}{N}\right) d t=\int_{0}^{\infty} w^{\prime}(t)^{N} d t
\end{aligned}
$$

Para verificar $(i i)$, note que $w(t)^{p}=\left(n^{N-\frac{1}{N}} \omega_{N}^{\frac{1}{N}}\right)^{\frac{N}{N-1}} v(r)^{p}=\alpha_{N} v(r)^{p}$. Como $r=R e^{-\frac{t}{N}}$, então $r^{N-1} d r=r^{N-1}\left(-\frac{r}{N} d t\right)=-\frac{r^{N}}{N} d t=-\frac{R^{N} e^{-t}}{N} d t$. Logo, fazendo a mudança de variáveis e usando coordenadas radiais

$$
\begin{aligned}
\int_{\Omega^{*}} e^{\alpha u^{* p}} d x & =\omega_{N-1} \int_{0}^{R} e^{\alpha v(r)^{p}} r^{N-1} d r=-\frac{\omega_{N-1} R^{N}}{N} \int_{\infty}^{0} e^{\frac{\alpha}{\alpha_{N}} w(t)^{p}} e^{-t} d t \\
& =\frac{\omega_{N-1} R^{N}}{N} \int_{0}^{\infty} e^{\beta w(t)^{p}-t} d t
\end{aligned}
$$

Finalmente, observe que $|\Omega|=\left|B_{R}(0)\right|=\frac{\omega_{N-1} R^{N}}{N}$. Portanto, de (2.18) e das identidades $(i)$ e $(i i)$, para obter o teorema é suficiente demonstrar que:

Sejam $w \in \mathcal{C}^{\infty}([0,+\infty))$ e $N \geq 2$, tais que

$$
w(0)=0, \quad w^{\prime} \geq 0, \quad \int_{0}^{\infty} w^{\prime}(t)^{N} d t \leq 1,
$$


então

$$
\int_{0}^{\infty} e^{\beta w^{p}-t} d t \leq C, \text { se } \beta \leq 1, \text { onde } \frac{1}{p}+\frac{1}{N}=1,
$$

onde $C$ é uma constante positiva que só depende de $N$.

O primeiro passo será provar que a integral em (2.20) é finita para cada valor de $\beta \leq 1$, e pode ser ilimitada para alguma função $w$ se $\beta>1$.

- Caso $\beta<1$

Como $w(0)=0$ e $w^{\prime} \geq 0$, então para cada $t>0$ temos

$$
\begin{aligned}
w(t) & =\int_{0}^{t} w^{\prime}(s) d s=\left\|w^{\prime}\right\|_{L^{1}([0, t])} \leq\|1\|_{L^{p}([0, t])}\left\|w^{\prime}\right\|_{L^{N}([0, t])} \\
& =\left(\int_{0}^{t} 1 d s\right)^{\frac{1}{p}}\left(\int_{0}^{t} w^{\prime}(s)^{N} d s\right)^{\frac{1}{N}} \\
& \leq t^{\frac{1}{p}}\left(\int_{0}^{\infty} w^{\prime}(s)^{N} d s\right)^{\frac{1}{N}} \leq t^{\frac{1}{p}}
\end{aligned}
$$

Logo, $w(t)^{p} \leq t$, para cada $t \geq 0$, e assim

$$
\int_{0}^{\infty} e^{\beta w^{p}-t} d t \leq \int_{0}^{\infty} e^{\beta t-t} d t=\left.\frac{e^{(\beta-1) t}}{\beta-1}\right|_{t=0} ^{t=\infty}=\frac{1}{1-\beta}
$$

- Caso $\beta=1$ Como $\int_{0}^{\infty} w^{\prime}(t)^{N} d t \leq 1$, então para cada $\epsilon>0$ existe $0<T=T(\epsilon)$, tal que

$$
\int_{T}^{\infty} w^{\prime}(t)^{N} d t<\epsilon
$$

Logo, se $t>T$

$$
\begin{aligned}
w(t)-w(T) & =\int_{T}^{t} w^{\prime}(s) d s=\left\|w^{\prime}\right\|_{L^{1}([T, t])} \leq\|1\|_{L^{p}([T, t])}\left\|w^{\prime}\right\|_{L^{N}([T, t])} \\
& =\left(\int_{T}^{t} 1 d s\right)^{\frac{1}{p}}\left(\int_{T}^{t} w^{\prime}(s)^{N} d s\right)^{\frac{1}{N}} \\
& \leq(t-T)^{\frac{1}{p}}\left(\int_{T}^{\infty} w^{\prime}(s)^{N} d s\right)^{\frac{1}{N}} \leq(t-T)^{\frac{1}{p}} \epsilon^{\frac{1}{N}} .
\end{aligned}
$$

Assim, para $t>T$, temos $\frac{w(t)}{t^{\frac{1}{p}}} \leq \frac{w(T)}{t^{\frac{1}{p}}}+\left(1-\frac{T}{t}\right)^{\frac{1}{p}} \epsilon^{\frac{1}{N}}$. E, portanto, como $\epsilon>0$ é 
arbitrário, temos

$$
\lim _{t \rightarrow \infty} \frac{w(t)}{t^{\frac{1}{p}}}=0, \quad \text { e, portanto, } \quad \lim _{t \rightarrow \infty} \frac{w(t)^{p}}{t}=0
$$

Por conseguinte, existe $t_{0}>0$, tal que $w(t)^{p}<\frac{t}{2}$, para todo $t \geq t_{0}$. Logo,

$$
\begin{aligned}
\int_{0}^{\infty} e^{w^{p}-t} d t & \leq \int_{0}^{t_{0}} e^{w^{p}-t} d t+\int_{t_{0}}^{\infty} e^{\frac{t}{2}-t} d t=\int_{0}^{t_{0}} e^{w^{p}-t} d t-\left.2 e^{-\frac{t}{2}}\right|_{t=t_{0}} ^{t=\infty} \\
& \leq \int_{0}^{t_{0}} e^{w^{p}-t} d t+2<\infty
\end{aligned}
$$

- Caso $\beta>1$

Defina $\eta=\min \{1, s\}$ e para $k>0$ considere a função

$$
w(t)=k^{1 / p} \eta\left(\frac{t}{k}\right)= \begin{cases}\frac{t}{k^{1 / N}}, & 0 \leq t \leq k, \\ k^{1 / p}, & k<t .\end{cases}
$$

Logo, $w(0)=0, w^{\prime} \geq 0 \mathrm{e} \int_{0}^{\infty} w^{\prime}(t)^{N} d t=\int_{0}^{k}\left(\frac{1}{k^{\frac{1}{N}}}\right)^{N} d t=\frac{1}{k} \int_{0}^{k} d t=1 \mathrm{E}$, portanto, para todo $k>0$ a função $w$ satisfaz as condições (2.19). Mas

$$
\begin{aligned}
\int_{0}^{\infty} e^{\beta w^{p}-t} d t & \geq \int_{k}^{\infty} e^{\beta w^{p}-t} d t=\int_{k}^{\infty} e^{\beta k-t} d t \\
& =e^{\beta k} \int_{k}^{\infty} e^{-t} d t=\left.e^{\beta k}\left(-e^{-t}\right)\right|_{t=k} ^{t=\infty}=e^{(\beta-1) k}
\end{aligned}
$$

Logo, se $k \rightarrow \infty$, tem-se $\int_{0}^{\infty} e^{\beta w^{p}-t} d t \rightarrow \infty$ portanto, a integral em (2.20) pode ser arbitrariamente grande desde que se tome $w \operatorname{com} k$ suficientemente grande.

Seja $w$ satisfazendo (2.19), pela desigualdade em (2.21), temos

$$
w(t) \leq t^{\frac{1}{p}}, \quad \text { para todo } t \geq 0
$$

Suponha que existe um ponto onde se satisfaz a desigualdade acima, isto é $w\left(t_{1}\right)=t_{1}^{1 / p}$, para algum ponto $t_{1}>0$. Logo, usando (2.21) todos os termos são iguais, isto é

$$
w\left(t_{1}\right)=\int_{0}^{t_{1}} w^{\prime}(t) d t=t_{1}^{1 / p}\left(\int_{0}^{t_{1}} w^{\prime}(t)^{N} d t\right)^{\frac{1}{N}}=t_{1}^{1 / p} .
$$


Donde obtemos que:

$$
\int_{0}^{t_{1}} w^{\prime}(t) d t=t_{1}^{1 / p}, \quad \int_{0}^{t_{1}} w^{\prime}(t)^{N} d t=1, \quad w^{\prime}(t)=0, \text { para } t>t_{1} .
$$

Disto, segue-se que $w(t)=t_{1}^{1 / p} \eta\left(\frac{t}{t_{1}}\right)$. Agora suponha que não se tem a igualdade (2.22) em algum punto. Como $w$ tem suporte compacto, existe $t_{1}>0$, tal que

$$
\max _{t>0} \frac{w(t)}{t^{1 / p}}=\frac{w\left(t_{1}\right)}{t_{1}^{1 / p}}<1
$$

E, portanto, sua N-ésima potência, satisfaz

$$
\max _{t>0} \frac{w(t)^{N}}{t^{N-1}}=\frac{w\left(t_{1}\right)^{N}}{t_{1}^{N-1}}=1-\delta, \quad \text { para algum } 0<\delta \leq 1 .
$$

Agora, façamos as mudança de variáveis $t=t_{1} s$ e $y(s)=\frac{w(t)}{t_{1}^{1 / p}}$. Como

$$
y(0)=0, \quad y^{\prime}(s)=w^{\prime}(t) t_{1}^{1 / N} \geq 0, \quad \text { para todo } s \geq 0,
$$

então,

$$
\int_{0}^{\infty} y^{\prime}(s)^{N} d s=\int_{0}^{\infty} w^{\prime}(t)^{N} t_{1} \frac{d t}{t_{1}}=\int_{0}^{\infty} w^{\prime}(t)^{N} d t \leq 1
$$

Além disso,

$$
\frac{y(s)^{N}}{s^{N-1}}=\frac{w\left(t_{1} s\right)^{N}}{\left(t_{1} s\right)^{N-1}}=\frac{w(t)^{N}}{t^{N-1}} \leq 1-\delta=\left(\frac{w\left(t_{1}\right)}{t_{1}^{p}}\right)^{N}, \text { para todo } s \geq 0 .
$$

Resumindo,

$$
y(0)=0, \quad \int_{0}^{\infty} y^{\prime}(s)^{N} d s \leq 1
$$

e para cada $s \geq 0$,

$$
y^{\prime}(s) \geq 0, \quad \frac{y(s)^{N}}{s^{N-1}} \leq 1-\delta=y_{1}^{N}
$$

onde $y_{1}=y(1)$.

Prova do Teorema 2.9 Elevando ao $\frac{1}{N-1}$ a desigualdade $\frac{w(t)^{N}}{t^{N-1}} \leq 1-\delta$, tem-se

$$
w(t)^{p} \leq(1-\delta)^{p-1} t, \quad \text { para todo } t \geq 0, \operatorname{com} p=\frac{N}{N-1} .
$$


Note que, se $0<\lambda<1$, a função $h(m)=(1-m)^{\lambda}+\lambda m$ definida no intervalo [0,1], possui um máximo no ponto 0 . Tomando em particular $\lambda=p-1$ e $m=\delta$, temos

$$
(1-\delta)^{p-1}+(p-1) \delta \leq 1
$$

Por conseguinte,

$$
(1-\delta)^{p-1} t-t \leq-(p-1) \delta t, \quad \text { para todo } t \geq 0
$$

Tome $0<\delta<\min \left\{\delta_{0}, \frac{1}{2 C_{4}}\right\}$ e considere $\Delta_{1}=\Delta t_{1}=\left\{t \in \mathbb{R}^{+}:\left|t-t_{1}\right|<C_{4} t_{1} \delta\right\}$.

Deste modo, $\frac{t_{1}}{2}<t_{1}-C_{4} \delta t_{1}$ e para cada $t \in \Delta_{1}$, temos $t_{1}-C_{4} \delta t_{1}<t$.

E, portanto,

$$
\frac{t_{1}}{2}<t, \text { para cada } t \in \Delta_{1} .
$$

Usando (2.23) e (2.24), temos

$$
\begin{aligned}
\int_{\Delta_{1}} e^{w(t)^{p}-t} d t & \leq \int_{\Delta_{1}} e^{(1-\delta)^{p-1} t-t} d t \leq \int_{\Delta_{1}} e^{-(p-1) \delta t} d t \\
& =2 C_{4} \delta t_{1} \max _{t \in \Delta_{1}}\left\{e^{-(p-1) \delta t}\right\}
\end{aligned}
$$

Usando (2.25), temos $\max _{t \in \Delta_{1}}\left\{e^{-(p-1) \delta t}\right\} \leq e^{\frac{-(p-1) \delta t_{1}}{2}}$. E, portanto,

$$
\begin{aligned}
\int_{\Delta_{1}} e^{w(t)^{p}-t} d t & \leq 2 C_{4} \delta t_{1} e^{\frac{-(p-1) \delta t_{1}}{2}} \\
& =\frac{4 C_{4}}{p-1}\left(\frac{(p-1) \delta t_{1}}{2}\right) e^{-\left(\frac{(p-1) \delta t_{1}}{2}\right)} \\
& \leq e^{\frac{-(p-1) \delta t_{1}}{2}} e^{-1}
\end{aligned}
$$

onde usamos que $x e^{-x} \leq e^{-1}$ para todo $x \geq 0$. Por outro lado,

$$
\begin{aligned}
t_{1} \int_{0}^{\infty} e^{-\frac{t_{1} \varphi(s)}{C_{5}}} d s & =t_{1}\left(\int_{0}^{1 / 2} e^{-\frac{t_{1} \varphi(s)}{C_{5}}} d s+\int_{1 / 2}^{1} e^{-\frac{t_{1} \varphi(s)}{C_{5}}} d s+\int_{1}^{\infty} e^{-\frac{t_{1} \varphi(s)}{C_{5}}} d s\right) \\
& =t_{1}\left(\int_{0}^{1 / 2} e^{-\frac{t_{1} s}{C_{5}}} d s+\int_{1 / 2}^{1} e^{-\frac{t_{1}(1-s)}{C_{5}}} d s+\int_{1}^{\infty} e^{-\frac{t_{1}(s-1)}{C_{5}}} d s\right) \\
& =t_{1}\left(\left[-\frac{C_{5}}{t_{1}} e^{-\frac{t_{1} s}{C_{5}}}\right]_{0}^{\frac{1}{2}}+\left[\frac{C_{5}}{t_{1}} e^{-\frac{t_{1}(1-s)}{C_{5}}}\right]_{\frac{1}{2}}^{1}+\left[-\frac{C_{5}}{t_{1}} e^{-\frac{t_{1}(s-1)}{C_{5}}}\right]_{1}^{\infty}\right) \\
& =3 C_{5}-2 C_{5} e^{-\frac{t_{1}}{2 C_{5}}} \leq 3 C_{5} .
\end{aligned}
$$


Observe que $t \notin \Delta_{1}$ se, e só se, $s \notin \Delta$. Logo pelo Lema 2.7 e o Lema 2.8 , temos

$$
w(t)^{p}-t=t_{1} y(s)^{p}-t_{1} s=t_{1}\left(y(s)^{p}-s\right) \leq t_{1}\left(z(s)^{p}-s\right) \leq-t_{1} \frac{\varphi(s)}{C_{5}} .
$$

Logo, por (2.27),

$$
\begin{aligned}
\int_{\mathbb{R}^{+} \backslash \Delta_{1}} e^{w(t)^{p}-t} d t & \leq \int_{\mathbb{R}^{+} \backslash \Delta_{1}} e^{-\frac{t-1 \varphi(s)}{C_{5}}} d t=t_{1} \int_{\mathbb{R}^{+} \backslash \Delta} e^{-\frac{t_{1} \varphi(s)}{C_{5}}} d s \\
& \leq t_{1} \int_{0}^{\infty} e^{-\frac{t_{1} \varphi(s)}{C_{5}}} d s \leq 3 C_{5} .
\end{aligned}
$$

E, portanto, se $0<\delta<\delta_{0}$ de (2.26) e (2.29)

$$
\int_{0}^{\infty} e^{w(t)^{p}-t} d t=\int_{\Delta_{1}} e^{w(t)^{p}-t} d t+\int_{\mathbb{R}^{+} \backslash \Delta_{1}} e^{w(t)^{p}-t} d t \leq 3 C_{5}+\frac{4 C_{4} e^{-1}}{p-1}=C_{6} .
$$

Se $\delta_{0} \leq \delta \leq 1$ usando (2.23) e a relação $w(t)^{p}-t \leq-(p-1) \delta t$, temos que

$$
\int_{0}^{\infty} e^{w(t)^{p}-t} d t \leq \int_{0}^{\infty} e^{-t(p-1) \delta t} d t=-\left.\frac{e^{-(p-1) \delta t}}{(p-1) \delta}\right|_{0} ^{\infty}=\frac{1}{(p-1) \delta} \leq \frac{1}{(p-1) \delta_{0}} .
$$

Portanto, para qualquer $0<\delta \leq 1$

$$
\int_{0}^{\infty} e^{w(t)^{p}-t} d t \leq C
$$

onde $C=C_{6}+\frac{1}{(p-1) \delta_{0}}$ e, portanto, $C=C(N)$. 


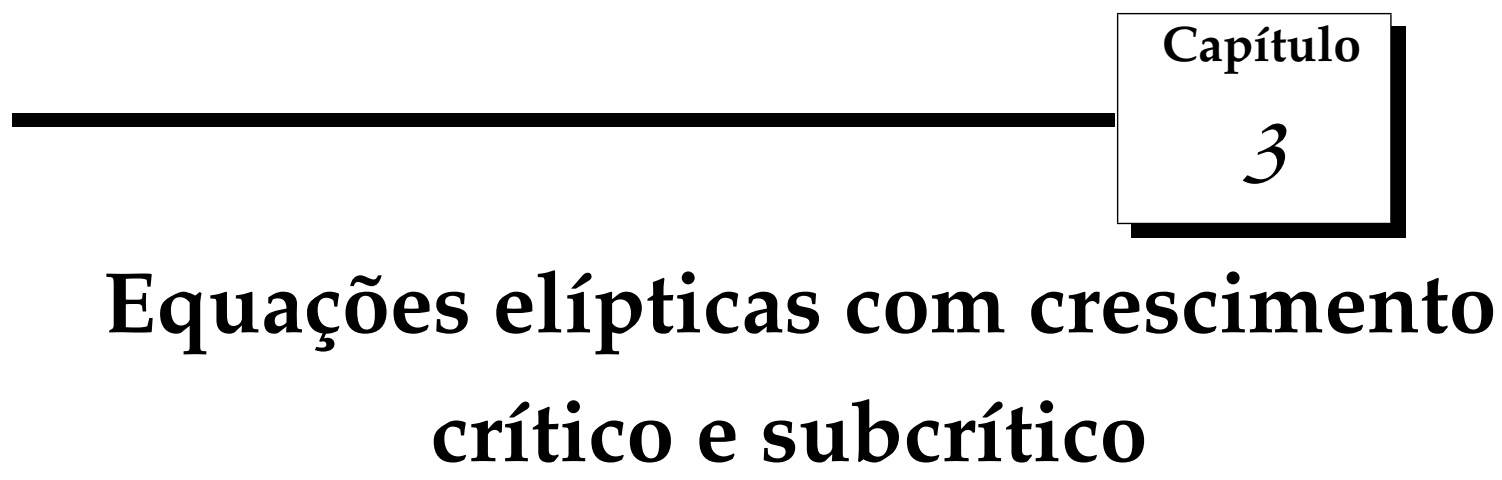

Neste capitulo, seguindo [7], estudaremos a existência de soluções não triviais do problema

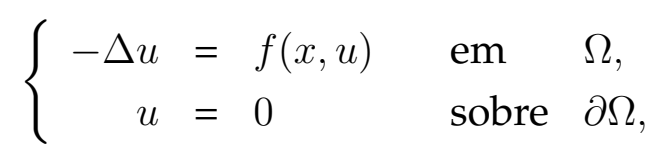

onde $\Omega$ é um domínio limitado em $\mathbb{R}^{2}$ e a função $f(x, s)$ possui crescimento maximal na variável $s$ que permite o uso de métodos variacionais. Dividiremos o estudo da existência de soluções em termos da noção de criticalidade dada a seguir.

Definição 3.1. Dizemos que $f: \bar{\Omega} \times \mathbb{R} \rightarrow \mathbb{R}$ possui

a) crescimento subcrítico, se

$$
\lim _{|t| \rightarrow \infty} \frac{|f(x, t)|}{e^{\alpha t^{2}}}=0, \quad \text { uniformemente em } \Omega, \quad \forall \alpha>0 .
$$

b) crescimento crítico, se existe $\alpha_{0}>0$ tal que

$$
\lim _{|t| \rightarrow \infty} \frac{|f(x, t)|}{e^{\alpha t^{2}}}= \begin{cases}0, & \text { uniformemente em } \Omega, \alpha_{0}<\alpha, \\ +\infty, & \text { uniformemente em } \Omega, 0<\alpha<\alpha_{0} .\end{cases}
$$

Admitiremos algumas condições na função $f$ que serão utilizadas neste capítulo: (H1) $f: \bar{\Omega} \times \mathbb{R} \rightarrow \mathbb{R}$ é contínua e $f(x, 0)=0$. 
(H2) Existem $t_{0}>0$ e $M>0$ tais que

$$
0<F(x, t)=\int_{0}^{t} f(x, s) d s \leq M|f(x, t)|, \forall|t| \geq t_{0}, \forall x \in \Omega
$$

(H3) $0<F(x, t) \leq \frac{1}{2} f(x, t) t, \forall t \in \mathbb{R} \backslash\{0\}, \forall x \in \Omega$.

Denotemos por $0<\lambda_{1}<\lambda_{2} \leq \lambda_{3} \ldots$, a sequência de autovalores do operador $\left(-\Delta, H_{0}^{1}(\Omega)\right)$ com condição de Dirichlet.

Teorema 3.2. (Caso Subcrítico, mínimo local em 0) Suponha (H1), (H2), (H3) e que $f$ possui crescimento subcrítico. Além disso, suponha que

$$
\lim _{t \rightarrow 0} \sup \frac{2 F(x, t)}{t^{2}}<\lambda_{1}, \quad \text { unif. em }(x, t) .
$$

Então, o problema (3.1) possui uma solução não trivial. Além disso, se $f(x, t)$ é uma função impar em $t$, então o problema possui infinitas soluções.

Teorema 3.3. (Caso Subcrítico, ponto sela em 0) Suponha (H1), (H2), (H3) e que f possui crescimento subcrítico. Além disso, suponha que

$$
\begin{gathered}
\exists \delta>0, \exists \lambda_{k} \leq \mu<\lambda_{k+1}, \text { tal que } F(x, t) \leq \frac{1}{2} \mu t^{2}, \quad \forall x \in \Omega, \forall|t| \leq \delta, \\
F(x, t) \geq \frac{1}{2} \lambda_{k} t^{2}, \quad \forall x \in \Omega, \forall t \in \mathbb{R} .
\end{gathered}
$$

Então o problema (3.1) possui uma solução não trivial. Além disso, se ao invés de (H6) supormos que $f(x, t)$ seja uma função impar em $t$, então o problema possui infinitas soluções.

Teorema 3.4. (Caso crítico, minimo local em 0) Suponha (H1), (H2), (H3) e que $f$ possui crescimento crítico, com constante de criticalidade $\alpha_{0}>0$. Além disso suponha (H4) e

$$
\lim _{t \rightarrow \infty} \frac{f(x, t) t}{e^{\alpha_{0} t^{2}}} \geq \beta, \quad \operatorname{com} \beta>\frac{2}{\alpha_{0} d^{2}},
$$

onde dé o raio da maior bola contida em $\Omega$. Então o problema (3.1) possui uma solução não trivial.

Exemplo 3.5. Uma função satisfazendo as hipóteses do Teorema 3.4 é dada pela função $f(x, t)=2 g(x) t e^{t^{2}}$, onde $g: \bar{\Omega} \rightarrow \mathbb{R}$ é uma função contínua, tal que $\sup _{x \in \bar{\Omega}} g(x)<\frac{\lambda_{1}}{2}$. Neste caso, $F(x, t)=g(x)\left(e^{t^{2}}-1\right)$. 


\subsection{A formulação variacional}

Nesta seção, consideraremos a seguinte condição

Existem contantes $C>0$ e $\beta>0$ tais que $|f(x, t)| \leq C e^{\beta t^{2}}, \quad \forall x \in \Omega, \forall t \in \mathbb{R}$.

Afirmação 3.6. Suponha que a função $f$ satisfaz a condição (H1). Então

(i) se possui crescimento crítico com constante de criticalidade $\alpha_{0}$, a condição (3.3) é satisfeita para $\beta>\alpha_{0}$.

(ii) se possui crescimento subcrítico, a condição (3.3) é satisfeita para $\beta>0$.

\section{Demonstração.}

(i) Seja $\beta>\alpha_{0}$, como $f$ possui crescimento crítico com constante de criticalidade $\alpha_{0}$, então

$$
\lim _{|t| \rightarrow \infty} \frac{|f(x, t)|}{e^{\beta t^{2}}}=0, \text { uniformemente em } \bar{\Omega} .
$$

Logo, dado $\epsilon=1$, existe $M>0$ tal que $\frac{|f(x, t)|}{e^{\beta t^{2}}} \leq 1, \forall x \in \bar{\Omega},|t| \geq M$. Seja $C_{1}=\max _{(x, t) \in \bar{\Omega} \times[-M, M]} \frac{|f(x, t)|}{e^{\beta t^{2}}}$ e considerando $C=\max \left\{1, C_{1}\right\}$, temos

$$
\frac{|f(x, t)|}{e^{\beta t^{2}}} \leq C, \quad \forall \in \Omega, \forall t \in \mathbb{R}
$$

(ii) Neste caso, pelo crescimento subcrítico, para cada $\beta>0$.

$$
\lim _{|t| \rightarrow \infty} \frac{|f(x, t)|}{e^{\beta t^{2}}}=0, \text { uniformemente em } \bar{\Omega} .
$$

Logo, basta fazer como na parte $(i)$.

Pelo Teorema 1.19, ou Teorema 2.9, temos

$$
e^{\alpha v^{2}} \in L^{1}(\Omega), \quad \forall v \in H_{0}^{1}(\Omega), \forall \alpha>0
$$

Logo,

$$
\int_{\Omega}|f(x, u)| d x \leq C \int_{\Omega} e^{\beta u^{2}} d x<+\infty, \quad \text { para todo } u \in H_{0}^{1}(\Omega) .
$$


Lema 3.7. Seja $\Omega$ um subconjunto aberto em $\mathbb{R}^{2}$ e $\left\{u_{n}\right\}$ uma sequência em $H_{0}^{1}(\Omega)$ que converge a u em $H_{0}^{1}(\Omega)$, então existem uma subsequência $\left\{u_{n_{k}}\right\}$ de $\left\{u_{n}\right\}$ e v em $H_{0}^{1}(\Omega)$ tais que

$$
\left|u_{n_{k}}(x)\right| \leq v(x) \text {, q.t.p. em } \Omega \quad \text { e } \quad u_{n_{k}}(x) \rightarrow u(x) \text {, q.t.p. em } \Omega \text {. }
$$

Demonstração. Considerando uma subsequência se for necessário, podermos supor que $u_{n} \rightarrow u$ q.t.p. em $\Omega$, logo para cada $k \geq 1$ escolha $w_{k}=u_{n_{k}}$ de $\left\{u_{n}\right\}$ tal que

$$
\left\|w_{k+1}-w_{k}\right\|_{H_{0}^{1}} \leq \frac{1}{2^{k}}
$$

Defina para cada $n \geq 1$,

$$
g_{n}(x)=\sum_{k=1}^{n}\left|w_{k+1}(x)-w_{k}(x)\right|
$$

Então, tem-se $g_{n} \in H_{0}^{1}(\Omega)$ e que $\left\|g_{n}\right\|_{H_{0}^{1}} \leq 1$, para cada $n \geq 1$. Por conseguinte, $\left\|g_{n}\right\|_{L^{2}} \leq 1$ e $\left\|\nabla g_{n}\right\|_{L^{2}} \leq 1$. Pelo Teorema da Convergência Monótona, temos $g_{n} \rightarrow q$ q.t.p. em $\Omega$, com $g \in L^{2}(\Omega)$. Além disso, pelo Teorema da Convergência Dominada, temos $\left\|g_{n}-g\right\|_{L^{2}} \rightarrow 0$ e usando o fato que $\left\{\left|\nabla g_{n}\right|\right\}$ é limitada em $L^{2}(\Omega)$, temos $g \in H_{0}^{1}(\Omega)$ (veja [5, Remark 4, Chapter 9]). Logo, para $l>k \geq 2$.

$$
\left|w_{l}(x)-w_{k}(x)\right| \leq\left|w_{l}(x)-w_{l-1}(x)\right|+\cdots+\left|w_{k+1}(x)-w_{k}(x)\right| \leq g(x)-g_{k-1}(x) \leq g(x) .
$$

Logo, fazendo $l \rightarrow+\infty$, tem-se $\left|u(x)-w_{k}(x)\right| \leq g(x)$ q.t.p. em $\Omega$. Por conseguinte, $\left|u_{n_{k}}(x)\right|=\left|w_{k}(x)\right| \leq v(x)$ q.t.p. em $\Omega, \operatorname{com} v=g+|u| \in H_{0}^{1}(\Omega)$.

Proposição 3.8. Suponha (H1), (H2) e (3.3). Então o funcional

$$
\begin{aligned}
\phi: H_{0}^{1}(\Omega) & \rightarrow \mathbb{R} \\
u & \mapsto \phi(u)=\int_{\Omega}|\nabla u|^{2} d x-\int_{\Omega} F(x, u) d x
\end{aligned}
$$

é uma aplicação de classe $\mathcal{C}^{1}\left(H_{0}^{1}(\Omega), \mathbb{R}\right)$ e

$$
\left\langle\phi^{\prime}(u), v\right\rangle=\int_{\Omega} \nabla u \nabla v d x-\int_{\Omega} f(x, u) v d x, \quad \text { para todo } v \in H_{0}^{1}(\Omega)
$$

Demonstração. Considere em $H_{0}^{1}(\Omega)$ a norma $\|u\|=\left(\int_{\Omega}|\nabla u|^{2} d x\right)^{1 / 2}$, além disso $H_{0}^{1}(\Omega)$ é um espaço de Hilbert com produto interno $\langle u, v\rangle=\left(\int_{\Omega} \nabla u \cdot \nabla v d x\right)^{1 / 2}$ para 
todo $u, v \in H_{0}^{1}(\Omega)$. Seja a aplicação $\gamma(u)=\int_{\Omega}|\nabla u|^{2} d x=\|u\|^{2}$. Logo, $\gamma$ é Gâteauxdiferenciável $H_{0}^{1}(\Omega)$. De fato, sejam $u, v \in H_{0}^{1}(\Omega)$. Então

$$
\begin{aligned}
\lim _{t \rightarrow 0} \frac{\gamma(u+t v)-\gamma(u)}{t} & =\lim _{t \rightarrow 0} \frac{1}{2}\left(\frac{\|u+t v\|^{2}-\|u\|^{2}}{t}\right) \\
& =\frac{1}{2} \lim _{t \rightarrow 0} \frac{\langle u+t v, u+t v\rangle-\langle u, u\rangle}{t} \\
& =\frac{1}{2} \lim _{t \rightarrow 0}[2\langle u, v\rangle+t\langle v, v\rangle] \\
& =\langle u, v\rangle .
\end{aligned}
$$

Asim,

$$
\gamma^{\prime}(u) v=\langle u, v\rangle=\int_{B} \nabla u \cdot \nabla v d x, \quad \forall u, v \in H_{0}^{1}(\Omega) .
$$

Para provar que $\gamma^{\prime}$ é continua, note que

$$
\begin{aligned}
& \gamma^{\prime}: H_{0}^{1}(\Omega) \rightarrow\left[H_{0}^{1}(\Omega)\right]^{*} \\
& u \quad \mapsto \quad \gamma^{\prime} u \quad: H_{0}^{1}(\Omega) \rightarrow \mathbb{R} \\
& v \quad \mapsto\left\langle\gamma^{\prime} u, v\right\rangle=\int_{\Omega} \nabla u \cdot \nabla v=\langle u, v\rangle .
\end{aligned}
$$

Pelo teorema da Representação de Riesz, $\gamma^{\prime}$ é isomorfismo isométrico, assim $\gamma^{\prime}$ é contínua. Portanto, usando a Proposição A.11, tem-se $\gamma \in \mathcal{C}^{1}\left(H_{0}^{1}(\Omega), \mathbb{R}\right)$.

Agora provemos que o seguinte funcional $\psi(u)=\int_{\Omega} F(x, u) d x$ é de classe $\mathcal{C}^{1}\left(H_{0}^{1}(\Omega), \mathbb{R}\right)$ e que

$$
\left\langle\psi^{\prime}(u), v\right\rangle=\int_{\Omega} f(x, u) v d x, \quad \text { para todo } v \in H_{0}^{1}(\Omega) .
$$

Vejamos primeiro que a função $\psi$ está bem definida. Pela desigualdade de Young, temos

$$
|F(x, t)|=\left|\int_{0}^{t} f(x, s) d s\right| \leq C \int_{0}^{|t|} e^{\beta s^{2}} d s \leq C e^{\beta t^{2}}|t| \leq \frac{C}{2}\left(e^{2 \beta t^{2}}+t^{2}\right) .
$$

E, portanto, usando (3.4) e a imersão $H_{0}^{1}(\Omega) \hookrightarrow L^{2}(\Omega)$

$$
|\psi(u)| \leq\left|\int_{\Omega}\right| F(x, u) \mid d x \leq \frac{C}{2} \int_{\Omega}\left(|u|^{2}+e^{2 \beta u^{2}}\right) d x<+\infty, \quad \text { para todo } u \in H_{0}^{1}(\Omega) .
$$

Sejam $u, v \in H_{0}^{1}(\Omega)$ e $0<|t|<1$, pelo Teorema do Valor Médio, temos

$$
\frac{F(x, u+t v)-F(x, u)}{t}=f(x, u+\theta t v) v, \quad \operatorname{com} 0<\theta<1 .
$$


Pela continuidade de $f$

$$
\lim _{t \rightarrow 0} \frac{F(x, u+t v)-F(x, u)}{t}=f(x, u) v .
$$

Além disso, usando (3.3) em (3.7)

$$
\begin{aligned}
\left|\frac{F(x, u+t v)-F(x, u)}{t}\right| & =|f(x, u+\theta t v) v| \leq C e^{\beta|u+t \theta v|^{2}}|v| \leq C e^{\beta(|u|+|v|)^{2}}|v| \\
& \leq \frac{C}{2}\left(e^{2 \beta(|u|+|v|)^{2}}+|v|^{2}\right) \in L^{1}(\Omega) .
\end{aligned}
$$

Pelo Teorema da Convergência Dominada

$$
\begin{aligned}
\psi^{\prime}(u) v & =\lim _{t \rightarrow 0} \frac{\psi(u+t v)-\psi(u)}{t}=\lim _{t \rightarrow 0} \int_{\Omega} \frac{F(x, u+t v)-F(x, u)}{t} d x \\
& =\int_{\Omega} \lim _{t \rightarrow 0} \frac{F(x, u+t v)-F(x, u)}{t} d x=\int_{\Omega} f(x, u) v d x .
\end{aligned}
$$

E, portanto, $\psi$ possui derivada de Gâteaux e $\psi^{\prime}(u) v=\int_{\Omega} f(x, u) v d x$, para todo $u, v \in$ $H_{0}^{1}(\Omega)$. Mostraremos agora a continuidade da derivada de Gâteaux. Seja $\left\{u_{n}\right\}$ uma sequência em $H_{0}^{1}(\Omega)$ tal que $u_{n} \rightarrow u$ em $H_{0}^{1}(\Omega)$, quando $n \rightarrow \infty$. Pelo Lema 3.7 existem uma subsequência $\left\{u_{n_{k}}\right\}$ de $\left\{u_{n}\right\}$ e $v \in H_{0}^{1}(\Omega)$ tais que

$$
\left|u_{n_{k}}(x)\right| \leq v(x) \quad \text { e } \quad u_{n_{k}}(x) \rightarrow u(x) \text {, q.t.p. em } \Omega
$$

E, portanto,

$$
\left|f\left(x, u_{n_{k}}\right)-f(x, u)\right|^{2} \leq C\left(e^{\beta v^{2}}+e^{\beta u^{2}}\right)^{2} \leq 2 C\left(e^{2 \beta v^{2}}+e^{2 \beta u^{2}}\right) \quad \in L^{1}(\Omega) .
$$

Pela continuidade de $f$, temos $\left(f\left(x, u_{n_{k}}\right)-f(x, u)\right)^{2} \rightarrow 0$, q.t.p. em $\Omega$. Pelo Teorema da Convergência Dominada de Lebesgue, temos

$$
\left\|f\left(\cdot, u_{n_{k}}\right)-f(\cdot, u)\right\|_{L^{2}}^{2}=\int_{\Omega}\left|f\left(x, u_{n_{k}}\right)-f(x, u)\right|^{2} d x \rightarrow 0, \quad \text { se } k \rightarrow+\infty
$$

Pela desigualdade de Schwarz, temos

$$
\left|\left\langle\psi^{\prime}\left(u_{n_{k}}\right)-\psi^{\prime}(u), v\right\rangle\right| \leq \int_{\Omega}\left|\left(f\left(x, u_{n_{k}}\right)-f(x, u)\right) \cdot v\right| d x \leq\left\|f\left(\cdot, u_{n_{k}}\right)-f(\cdot, u)\right\|_{L^{2}}\|v\|_{L^{2}} .
$$


Então,

$$
\| \psi^{\prime}\left(u_{n_{k}}\right)-\psi^{\prime}\left(u\left\|=\sup _{\|v\|_{L^{2}} \leq 1}\left|<\psi^{\prime}\left(u_{n_{k}}\right)-\psi^{\prime}(u), v>\right| \leq\right\| f\left(\cdot, u_{n_{k}}\right)-f(\cdot, u) \|_{L^{2}} \rightarrow 0 .\right.
$$

Por conseguinte, qualquer subsequência de $\left\{\psi^{\prime}\left(u_{n}\right)\right\}$ converge a $\psi^{\prime}(u)$. O que prova que $\psi^{\prime}$ é contínua. Logo, pela Proposição A.11, tem-se $\psi \in \mathcal{C}^{1}\left(H_{0}^{1}(\Omega), \mathbb{R}\right)$, Deste modo, $\phi=\gamma-\psi \in \mathcal{C}^{1}\left(H_{0}^{1}(\Omega), \mathbb{R}\right)$.

Lema 3.9. Suponha que f satisfaz (H1)-(H3). Então

(a) Existe uma constante $C_{0}>0$ tal que $F(x, t) \geq C_{0} e^{|t| / M}, \forall|t| \geq t_{0}$.

(b) Dado $\epsilon>0$ existe $t_{\epsilon}>0$ tal que

$$
F(x, t) \leq \epsilon f(x, t) t, \quad \forall x \in \Omega, \forall|t| \geq t_{\epsilon} .
$$

(c) Existe $\bar{M}>0$ tal que $F(x, t) \leq \bar{M}|f(x, t)|, \forall x \in \Omega, \forall t \in \mathbb{R}$.

Demonstração. Para provar $(a)$ analisemos separadamente os casos $t \geq t_{0}$ e $t \leq-t_{0}$.

- Caso $t \geq t_{0}$.

Por $(\mathrm{H} 3) f(x, s)>0$, para todo $s \geq t_{0}$. Além disso, por $(\mathrm{H} 2)$, temos

$$
\int_{t_{0}}^{t} \frac{1}{M} d s \leq \int_{t_{0}}^{t} \frac{|f(x, s)|}{F(x, s)} d s=\int_{t_{0}}^{t} \frac{f(x, s)}{F(x, s)} d s .
$$

Logo,

$$
\frac{1}{M}\left(t-t_{0}\right) \leq \ln \left(\frac{F(x, t)}{F\left(x, t_{0}\right)}\right)
$$

Assim,

$$
\frac{F\left(x, t_{0}\right)}{e^{t_{0} / M}} e^{t / M} \leq F(x, t)
$$

Como $F$ é contínua e positiva em $\bar{\Omega} \times\left\{t_{0}\right\}$ então $0<C_{1}=\inf _{x \in \bar{\Omega}} \frac{F\left(x, t_{0}\right)}{e^{t_{0} / M}}$. Por conseguinte,

$$
C_{1} e^{t / M} \leq F(x, t), \quad \text { para todo } t \geq t_{0}
$$

- Caso $t \leq-t_{0}$.

Neste caso, temos $f(x, s)<0$, para todo $\leq-t_{0}$, por $(\mathrm{H} 2)$, temos

$$
\int_{t}^{-t_{0}} \frac{1}{M} d s \leq \int_{t}^{-t_{0}} \frac{|f(x, s)|}{F(x, s)} d s=-\int_{t}^{-t_{0}} \frac{f(x, s)}{F(x, s)} d s .
$$


E, portanto,

$$
C_{2} e^{-t / M} \leq F(x, t), \quad \text { para todo } t \leq-t_{0},
$$

onde, $0<C_{2}=\inf _{x \in \bar{\Omega}} \frac{e^{-t_{0} / M}}{F\left(x,-t_{0}\right)}$.

Para concluir a prova basta tomar $C_{0}=\min \left\{C_{1}, C_{2}\right\}$.

Provemos $(b)$. Dado $\epsilon>0$, considere $t_{\epsilon}=\max \left\{t_{0}, \frac{M}{\epsilon}\right\}$, usando (H2) para $|t| \geq t_{\epsilon}$ temos

$$
\left|\frac{F(x, t)}{f(x, t) t}\right| \leq \frac{M|f(x, t)|}{|f(x, t) t|}=\frac{M}{|t|} \leq \frac{M}{t_{\epsilon}} \leq \epsilon .
$$

Logo,

$$
F(x, t) \leq \epsilon f(x, t) t, \quad \forall x \in \Omega, \forall|t| \geq t_{\epsilon} .
$$

Pois de (H3) $F(x, t)>0$ e $f(x, t) t>0, \forall t \neq 0$.

Por fim provemos $(c)$. Note que de $(\mathrm{H} 3)$,

$$
F(x, t) \leq \frac{1}{2} f(x, t) t \leq \frac{t_{0}}{2}|f(x, t)|, \quad \forall|t| \leq t_{0}
$$

Então usando (H2), basta tomar $\bar{M}=\max \left\{M, \frac{t_{0}}{2}\right\}$.

Lema 3.10. (Lema de Lions) Sejam $\left\{v_{n}\right\}$ uma sequência em $H_{0}^{1}(\Omega), v \in H_{0}^{1}(\Omega)$, com $\|v\|<1$, $\left\|v_{n}\right\|=1$ para todo $n \geq 1$ e $v_{n} \rightarrow$ vem $H_{0}^{1}(\Omega)$, então

$$
\sup _{n \geq 1} \int_{\Omega} e^{4 \pi p v_{n}^{2}} d x<\infty, \quad \text { se } \quad p<\frac{1}{1-\|v\|^{2}}
$$

Demonstração. Sejam $p_{1}>p$ e $\epsilon>0$ tais que $p<p_{1}\left(1+\frac{1}{\epsilon}\right)<\frac{1}{1-\|v\|^{2}}$. Das hipóteses, temos

$$
\left\|v_{n}-v\right\|^{2}=\left\|v_{n}\right\|^{2}-2 \int_{\Omega} \nabla v_{n} \nabla v d x+\|v\|^{2} \rightarrow 1-\|v\|^{2}, \quad \text { se } n \rightarrow \infty
$$

Logo,

$$
p_{1}\left(1+\frac{1}{\epsilon}\right)<\lim _{n \rightarrow \infty} \frac{1}{\left\|v_{n}-v\right\|^{2}}
$$

E, portanto, existe $n_{0} \in \mathbb{N}$, tal que $p_{1}\left(1+\frac{1}{\epsilon}\right)<\frac{1}{\left\|v_{n}-v\right\|^{2}}$, para todo $n \geq n_{0}$. Pelo 
Teorema 2.9, existe uma constante $C>0$ tal que todo $n \geq n_{0}$

$$
\int_{\Omega} e^{4 \pi p_{1}\left(1+\frac{1}{\epsilon}\right)\left(v_{n}-v\right)^{2}} d x \leq \int_{\Omega} e^{4 \pi\left(\frac{v_{n}-v}{\left\|v_{n}-v\right\|}\right)^{2}} d x \leq C .
$$

Dados $a, b \in \mathbb{R}$, tem-se $a^{2} \leq\left(1+\frac{1}{\epsilon}\right)(a-b)^{2}+(1+\epsilon) b^{2}$. Logo, tomando para cada $n \geq 1$, $a=v_{n}(x)$ e $b=v(x)$, temos

$$
v_{n}^{2} \leq\left(1+\frac{1}{\epsilon}\right)\left(v_{n}-v\right)+(1+\epsilon) v^{2}, \quad \text { para todo } n \geq 1 .
$$

Como $\frac{1}{p}>\frac{1}{p_{1}}$, seja $p_{2}>0$ tal que $\frac{1}{p}=\frac{1}{p_{1}}+\frac{1}{p_{2}}$. Pela desigualdade de Hölder e de (3.9)

$$
\begin{aligned}
\int_{\Omega} e^{4 \pi p v_{n}^{2}} d x & \leq \int_{\Omega} e^{4 \pi p\left(\left(1+\frac{1}{\epsilon}\right)\left(v_{n}-v\right)+(1+\epsilon) v^{2}\right)} d x=\int_{\Omega} e^{4 \pi p\left(1+\frac{1}{\epsilon}\right)\left(v_{n}-v\right)} e^{4 \pi p(1+\epsilon) v^{2}} d x \\
& \leq\left(\int_{\Omega} e^{4 \pi p_{1}\left(1+\frac{1}{\epsilon}\right)\left(v_{n}-v\right)} d x\right)^{p / p_{1}}\left(\int_{\Omega} e^{4 \pi p_{2}(1+\epsilon) v^{2}} d x\right)^{p / p_{2}} .
\end{aligned}
$$

Logo, usando (3.4) e(3.8), temos

$$
\int_{\Omega} e^{4 \pi p v_{n}^{2}} d x \leq C^{p / p_{1}}\left(\int_{\Omega} e^{4 \pi p_{2}(1+\epsilon) v^{2}} d x\right)^{p / p_{2}}<\infty, \quad \text { para todo } n \geq 1 .
$$

Proposição 3.11. Suponha (H1), (H2) e (3.3), então o funcional $\phi$ satisfaz a condição $(P S)_{c}$ para $c<\frac{2 \pi}{\beta}$.

Demonstração. Seja $c<\frac{2 \pi}{\beta}$ e $\left\{u_{n}\right\}$ uma sequência tal que

$$
\phi\left(u_{n}\right) \rightarrow c \quad \text { e }\left\|\phi^{\prime}\left(u_{n}\right)\right\|_{H^{-1}} \rightarrow 0, \quad \text { quando } n \rightarrow \infty
$$

isto é,

$$
\begin{gathered}
\frac{1}{2} \int_{\Omega}\left|\nabla u_{n}\right|^{2} d x-\int_{\Omega} F\left(x, u_{n}\right) d x \rightarrow c . \\
\left|\int_{\Omega} \nabla u_{n} \nabla v-f\left(x, u_{n}\right) v d x\right| \leq \epsilon_{n}\|v\|, \quad \text { para todo } v \in H_{0}^{1}(\Omega),
\end{gathered}
$$

onde $\epsilon_{n}=\left\|\phi^{\prime}\left(u_{n}\right)\right\|_{H^{-1}} \rightarrow 0, \quad$ quando $n \rightarrow \infty$. 
De (3.11), para $0<\epsilon<\frac{1}{2}$ existe $n_{0} \in \mathbb{N}$, tal que $\epsilon_{n}<1$, para todo $n \geq n_{0} \mathrm{e}$

$$
\left.\left|\frac{1}{2} \int_{\Omega}\right| \nabla u_{n}\right|^{2} d x-\int_{\Omega} F\left(x, u_{n}\right) d x-c \mid \leq \epsilon, \quad \text { para todo } n \geq n_{0} .
$$

Logo, usando a parte $(b)$ do Lema 3.9 para cada $n \geq n_{0}$, temos

$$
\begin{aligned}
\frac{1}{2}\left\|u_{n}\right\|^{2} & \leq \epsilon+c+\int_{\Omega} F\left(x, u_{n}\right) d x=\epsilon+c+\int_{\left\{\left|u_{n}\right|<t_{\epsilon}\right\}} F\left(x, u_{n}\right) d x+\int_{\left\{\left|u_{n}\right| \geq t_{\epsilon}\right\}} F\left(x, u_{n}\right) d x \\
& \leq \epsilon+c+\sup _{(x, t) \in \bar{\Omega} \times\left[0, t_{\epsilon}\right]}|F(x, t)| \int_{\left\{\left|u_{n}\right|<t_{\epsilon}\right\}} d x+\epsilon \int_{\left\{\left|u_{n}\right| \geq t_{\epsilon}\right\}} f\left(x, u_{n}\right) u_{n} d x \\
& \leq \epsilon+c+\sup _{(x, t) \in \bar{\Omega} \times\left[0, t_{\epsilon}\right]}|F(x, t)||\Omega|+\epsilon \int_{\Omega} f\left(x, u_{n}\right) u_{n} d x \\
& =c_{\epsilon}+\epsilon \int_{\Omega} f\left(x, u_{n}\right) u_{n} d x .
\end{aligned}
$$

Tomando $v=u_{n}$ para cada $n \geq n_{0}$ em (3.12), temos

$$
\left.\left|\int_{\Omega}\right| \nabla u_{n}\right|^{2}-f\left(x, u_{n}\right) u_{n} d x \mid \leq \epsilon_{n}\left\|u_{n}\right\|
$$

Disto e por (H3), temos

$$
\int_{\Omega} f\left(x, u_{n}\right) u_{n} d x \leq \epsilon_{n}\left\|u_{n}\right\|+\left\|u_{n}\right\|^{2}, \quad n \geq n_{0} .
$$

Combinando (3.13) e (3.14), temos

$$
\frac{\left\|u_{n}\right\|^{2}}{2} \leq c_{\epsilon}+\epsilon \epsilon_{n}\left\|u_{n}\right\|+\epsilon\left\|u_{n}\right\|^{2}, \quad n \geq n_{0}
$$

Como $\epsilon_{n}<1$, para todo $n \geq n_{0}$,

$$
\left(\frac{1}{2}-\epsilon\right)\left\|u_{n}\right\|^{2} \leq c_{\epsilon}+\epsilon\left\|u_{n}\right\|, \quad \text { para todo } n \geq n_{0}
$$

Suponha que $\left\{\left\|u_{n}\right\|\right\}_{n \geq n_{0}}$ não seja limitada em $\mathbb{R}$, então existe uma subsequência $\left\{\left\|u_{n_{k}}\right\|\right\}$ de $\left\{\left\|u_{n}\right\|\right\}_{n \geq n_{0}}$ tal que $\left\|u_{n_{k}}\right\| \rightarrow \infty$, quando $k \rightarrow \infty$. E, portanto, de (3.15)

$$
\left(\frac{1}{2}-\epsilon\right) \leq \frac{c_{\epsilon}}{\left\|u_{n_{k}}\right\|^{2}}+\frac{\epsilon}{\left\|u_{n_{k}}\right\|} \rightarrow 0, \quad \text { quando } k \rightarrow+\infty .
$$


Contradizendo o fato que $\epsilon<\frac{1}{2}$, então $\left\{u_{n}\right\}_{n \geq n_{0}}$ é limitada em $H_{0}^{1}(\Omega)$ e, portanto, $\left\{u_{n}\right\}$ é uma sequência limitada em $H_{0}^{1}(\Omega)$. Além disso, note que de (3.11) a sequência $\left\{\frac{\left\|u_{n}\right\|^{2}}{2}-\int_{\Omega} F\left(x, u_{n}\right) d x\right\}$ é limitada, e por conseguinte $\left\{\int_{\Omega} F\left(x, u_{n}\right) d x\right\}$ é uma sequência limitada, também de (3.14), tem-se que a sequência $\left\{\int_{\Omega} f\left(x, u_{n}\right) u_{n} d x\right\}$ é limitada. Então existe $C_{0}>0$ tal que

$$
\left\|u_{n}\right\| \leq C_{0}, \quad 0 \leq \int_{\Omega} f\left(x, u_{n}\right) u_{n} \leq C_{0}, \quad 0 \leq \int_{\Omega} F\left(x, u_{n}\right) \leq C_{0}
$$

onde as integrais são positivas pela condição (H3). Sendo $\left\{u_{n}\right\}$ uma sequência limitada no espaço reflexivo $H_{0}^{1}(\Omega)$ e da imersão compacta $H_{0}^{1}(\Omega) \hookrightarrow L^{q}(\Omega)$, para todo $q \geq 1$, tomando uma subsequência se for necessário, podemos supor que existe $u \in H_{0}^{1}(\Omega)$ tal que

$$
u_{n} \rightarrow u \text { em } H_{0}^{1}(\Omega), \quad u_{n} \rightarrow u \text { em } L^{q}(\Omega), \text { para todo } q \geq 1, \quad \text { e } u_{n} \rightarrow u \text { q.t.p. em } \Omega .
$$

Pelo Lema B.4, temos

$$
f\left(x, u_{n}\right) \rightarrow f(x, u) \quad \text { em } L^{1}(\Omega)
$$

Da continuidade de $F$ e da parte $(c)$ do Lema 3.9

$$
F\left(x, u_{n}\right) \rightarrow F(x, u) \text {, q.t.p. em } \Omega, \quad \text { e }\left|F\left(x, u_{n}\right)\right| \leq \bar{M}\left|f\left(x, u_{n}\right)\right|, \forall x \in \Omega, \forall n \geq 1 \text {. }
$$

Pelo Teorema da Convergência Dominada generalizada, temos

$$
F\left(x, u_{n}\right) \rightarrow F(x, u), \quad \text { em } L^{1}(\Omega), \quad \text { se } n \rightarrow \infty .
$$

Logo, de (3.11) e (3.18), quando $n \rightarrow \infty$, temos

$$
\frac{\left\|u_{n}\right\|^{2}}{2}=\left(\int_{\Omega} \frac{\left|\nabla u_{n}\right|^{2}}{2} d x-\int_{\Omega} F\left(x, u_{n}\right) d x\right)+\int_{\Omega} F\left(x, u_{n}\right) d x \rightarrow c+\int_{\Omega} F(x, u) d x,
$$

$\log \mathrm{O}$

$$
\lim _{n \rightarrow \infty}\left\|u_{n}\right\|^{2}=2\left(c+\int_{\Omega} F(x, u) d x\right) .
$$

Usando (3.12) com $v=u_{n}$ e o fato de que $\left\{\left\|u_{n}\right\|\right\}$ é limitada, resulta

$$
\left.\left|\int_{\Omega}\right| \nabla u_{n}\right|^{2}-f\left(x, u_{n}\right) u_{n} d x \mid \leq \epsilon_{n}\left\|u_{n}\right\| \rightarrow 0, \quad \text { quando } n \rightarrow \infty
$$


e, portanto,

$$
\lim _{n \rightarrow \infty}\left\|u_{n}\right\|^{2}=\lim _{n \rightarrow \infty} \int_{\Omega} f\left(x, u_{n}\right) u_{n} d x .
$$

Da condição (H3), temos

$$
0 \leq \int_{\Omega} F\left(x, u_{n}\right) d x \leq \frac{1}{2} \int_{\Omega} f\left(x, u_{n}\right) u_{n} d x \quad \forall n \geq 1
$$

Logo, tomando o limite quando $n \rightarrow \infty$ e usando (3.18) , (3.19) e (3.20)

$$
0 \leq \int_{\Omega} F(x, u) d x \leq c+\int_{\Omega} F(x, u) d x
$$

Donde concluímos que $c \geq 0$, isto quer dizer que não existem sequências de PalaisSmale para valores negativos.

Afirmação 1. $\phi(u) \geq 0$.

De fato, dada $\psi \in C_{0}^{\infty}(\Omega)$, então

$$
\begin{aligned}
\int_{\Omega}(\nabla u \nabla \psi-f(x, u) \psi) d x= & \int_{\Omega}\left(\nabla u \nabla \psi-\nabla u_{n} \nabla \psi\right) d x+\int_{\Omega}\left(\nabla u_{n} \nabla \psi-f\left(x, u_{n}\right) \psi\right) d x+ \\
& +\int_{\Omega}\left(f\left(x, u_{n}\right)-f(x, u)\right) \psi d x
\end{aligned}
$$

Mostremos que cada integral do lado direito tem limite igual a zero, e portanto a integral do lado esquerdo é zero. Como $u_{n} \rightarrow u$ em $H_{0}^{1}(\Omega)$, temos $\int_{\Omega}(\nabla u \nabla \psi-$ $\left.\nabla u_{n} \nabla \psi\right) d x \rightarrow 0$, quando $n \rightarrow \infty$. Pelo Lema B.4, temos

$$
\int_{\Omega}\left(f\left(x, u_{n}\right)-f(x, u)\right) \psi d x \mid \leq\left\|f\left(x, u_{n}\right)-f(x, u)\right\|_{L^{1}}\|\psi\|_{L^{\infty}} \rightarrow 0, \quad \text { quando } n \rightarrow \infty \text {. }
$$

Combinando isto com (3.12), para $v=\psi$, temos

$$
\int_{\Omega} \nabla u \nabla \psi=\int_{\Omega} f(x, u) \psi d x, \quad \text { para todo } \psi \in C_{0}^{\infty}(\Omega)
$$

Pela densidade de $\mathcal{C}_{0}^{\infty}(\Omega)$ em $H_{0}^{1}(\Omega)$, temos $\int_{\Omega}|\nabla u|^{2} d x=\int_{\Omega} f(x, u) u d x$

Além disso, de (H3), temos

$$
\int_{\Omega} F(x, u) d x \leq \frac{1}{2} \int_{\Omega} f(x, u) u d x=\int_{\Omega} \frac{|\nabla u|^{2}}{2} d x,
$$


assim

$$
\phi(u)=\int_{\Omega} \frac{|\nabla u|^{2}}{2}-\int_{\Omega} F(x, u) d x \geq 0 .
$$

Agora para provar a existência de uma subsequência convergente, analisaremos os seguintes casos:

- Caso 1. $c=0$.

Usando que $u_{n} \rightarrow u$ em $H_{0}^{1}(\Omega)$ e (3.19), obtemos

$$
\|u\|^{2} \leq \liminf _{n \rightarrow \infty}\left\|u_{n}\right\|^{2}=\lim _{n \rightarrow \infty}\left\|u_{n}\right\|^{2}=2 \int_{\Omega} F(x, u) d x .
$$

Logo,

$$
0 \leq \phi(u)=\frac{\|u\|^{2}}{2}-\int_{\Omega} F(x, u) d x \leq \int_{\Omega} F(x, u) d x-\int_{\Omega} F(x, u) d x=0 .
$$

E, portanto , $\phi(u)=0$ e de (3.19), tem-se

$$
\|u\|^{2}=2 \int_{\Omega} F(x, u) d x=\lim _{n \rightarrow \infty}\left\|u_{n}\right\|^{2} .
$$

Como temos $u_{n} \rightarrow u$ em $H_{0}^{1}(\Omega)$, concluímos

$$
u_{n} \rightarrow u, \quad \text { em } H_{0}^{1}(\Omega),
$$

é a condição $(P S)_{c}$ é satisfeita.

- Caso 2. $c \neq 0, u=0$.

Primeiro provaremos a seguinte afirmação.

Afirmação 2. Existem $q>1, C_{1}>0$ e $n_{0} \in \mathbb{N}$ tais que

$$
\int_{\Omega}\left|f\left(x, u_{n}\right)\right|^{q} \leq C_{1}, \quad \forall n \geq n_{0}
$$

De fato, como $c<\frac{2 \pi}{\beta}$, existe $\epsilon>0$ tal que $2 c+\epsilon<\frac{4 \pi}{\beta}$ e seja $q>1$ tal que 
$q(2 c+\epsilon)<\frac{4 \pi}{\beta}$. Como $u=0$, então $F(x, 0)=0$ e assim

$$
\lim _{n \rightarrow \infty}\left\|u_{n}\right\|^{2}=2\left(c+\int_{\Omega} F(x, 0) d x\right)=2 c .
$$

Então, existe $n_{0} \in \mathbb{N}$, tal que $\left\|u_{n}\right\|^{2}<2 c+\epsilon$, para todo $n \geq n_{0}$. Logo

$$
q \beta\left\|u_{n}\right\|^{2} \leq q \beta(2 c+\epsilon)<4 \pi, \quad \text { para todo } n \geq n_{0} .
$$

Pelo Teorema 2.9, existe $C_{0}$ tal que para todo $n \geq n_{0}$, tem-se

$$
\begin{aligned}
\int_{\Omega}\left|f\left(x, u_{n}\right)\right|^{q} & \leq C \int_{\Omega} e^{q \beta u_{n}^{2}} d x=C \int_{\Omega} e^{q \beta u_{n}^{2}\left(\frac{u_{n}}{\left\|u_{n}\right\|}\right)^{2}} d x \\
& \leq C \int_{\Omega} e^{q \beta(2 c+\epsilon)\left(\frac{u_{n}}{\left\|u_{n}\right\|}\right)^{2}} d x \leq C C_{0}=C_{1} .
\end{aligned}
$$

Logo, tomando $v=u_{n}$ para todo $n \geq 1$ em (3.12) e de (3.16), obtemos

$$
\left|\left\|u_{n}\right\|^{2}-\int_{\Omega} f\left(x, u_{n}\right) u_{n} d x\right| \leq \epsilon_{n}\left\|u_{n}\right\| \leq C_{0} \epsilon_{n}
$$

então

$$
\left\|u_{n}\right\|^{2} \leq C_{0} \epsilon_{n}+\int_{\Omega} f\left(x, u_{n}\right) u_{n} d x, \quad \text { para todo } n \geq 1 .
$$

Pela afirmação 2 para todo $n \geq n_{0}, f\left(\cdot, u_{n}\right) \in L^{q}(\Omega)$ e por (3.17) $u_{n} \rightarrow u=0$ em $L^{q^{\prime}}(\Omega)$. Pela desigualdad de Hölder, tem-se

$$
\begin{aligned}
0 \leq \int_{\Omega} f\left(x, u_{n}\right) u_{n} d x & \leq\left(\int_{\Omega}\left|f\left(x, u_{n}\right)\right|^{q} d x\right)^{1 / p}\left(\int_{\Omega}\left|u_{n}\right|^{q^{\prime}} d x\right)^{1 / q^{\prime}} \\
& \leq C_{1}^{1 / p}\left\|u_{n}\right\|_{L^{q^{\prime}}} \rightarrow 0, \text { quando } n \rightarrow \infty .
\end{aligned}
$$

Usando isto em (3.21), temos $\lim _{n \rightarrow \infty}\left\|u_{n}\right\|=0$ e de (3.19), temos

$$
0=\lim _{n \rightarrow \infty}\left\|u_{n}\right\|=2\left(c+\int_{\Omega} F(x, 0) d x\right)=2 c,
$$

donde temos uma contradição, pois estamos supondo que $c \neq 0, \operatorname{logo}$, o caso 2 não é possível.

- Caso $3 c \neq 0, u \neq 0$. 
Pela convergência fraca de $u_{n} \rightarrow u$ em $H_{0}^{1}(\Omega)$, temos

$$
0 \leq \phi(u)=\frac{\|u\|^{2}}{2}-\int_{\Omega} F(x, u) d x \leq \frac{1}{2} \lim _{n \rightarrow \infty}\left\|u_{n}\right\|^{2}-\int_{\Omega} F(x, u) d x=c
$$

então $0 \leq \phi(u) \leq c$.

Se $\phi(u)=c$, de (3.22), temos que $\|u\|=\lim _{n \rightarrow \infty}\left\|u_{n}\right\|$ e usando o fato que $u_{n} \rightarrow u$ em $H_{0}^{1}(\Omega)$, resulta

$$
\lim _{n \rightarrow \infty} u_{n}=u, \quad \text { em } H_{0}^{1}(\Omega),
$$

e condição $(P S)_{c}$ é satisfeita.

Para $0 \leq \phi(u)<c$ precisamos da seguinte afirmação.

Afirmação 3. Existem $q>1, C_{2}>0$ e $n_{0} \in \mathbb{N}$, tais que

$$
\int_{\Omega}\left|f\left(x, u_{n}\right)\right|^{q} \leq C_{2}, \quad \text { para todo } n \geq n_{0} .
$$

De fato, como $0<c-\phi(u)<\frac{2 \pi}{\beta}$, logo, $\beta<\frac{2 \pi}{c-\phi(u)}$, além disso $0<$ $\frac{2 \pi}{c+\int_{\Omega} F(x, u) d x}, \log$ existe $p>0$ tal que

$$
\beta<p \frac{2 \pi}{c+\int_{\Omega} F(x, u) d x}<\frac{2 \pi}{c-\phi(u)} .
$$

Usando (3.19), tem-se

$$
\beta \lim _{n \rightarrow \infty}\left\|u_{n}\right\|^{2}=2 \beta\left(c+\int_{\Omega} F(x, u) d x\right)<4 \pi p<4 \pi \frac{c+\int_{\Omega} F(x, u) d x}{c-\phi(u)} .
$$

Seja $q>1$, tal que

$$
q \beta \lim _{n \rightarrow \infty}\left\|u_{n}\right\|^{2}<4 \pi p<4 \pi \frac{c+\int_{\Omega} F(x, u) d x}{c-\phi(u)} .
$$

Por conseguinte, existe $n_{0} \in \mathbb{N}$, tal que

$$
q \beta\left\|u_{n}\right\|^{2} \leq 4 \pi p<4 \pi \frac{c+\int_{\Omega} F(x, u) d x}{c-\phi(u)}, \quad \text { para todo } n \geq n_{0} .
$$

Dado que $\lim _{n \rightarrow \infty}\left\|u_{n}\right\| \neq 0$, podemos supor que $\left\|u_{n}\right\| \neq 0$, para todo $n \geq n_{0}$. 
Definindo

$$
v_{n}=\frac{u_{n}}{\left\|u_{n}\right\|}, \quad v=\frac{u}{\sqrt{2\left(c+\int_{\Omega} F(x, u) d x\right)}} .
$$

Note que,

$$
\|v\|<1, \quad\left\|v_{n}\right\|=1, \text { para todo } n \geq n_{0}, \quad \text { e } \quad \frac{c+\int_{\Omega} F(x, u) d x}{c-\phi(u)}=\frac{1}{1-\|v\|^{2}}
$$

Também, como temos que $u_{n} \rightarrow u$ em $H_{0}^{1}(\Omega)$ e $\left.\left\|u_{n}\right\| \rightarrow \sqrt{2\left(c+\int_{\Omega} F(x, u) d x\right.}\right)$, então $v_{n} \rightarrow v$ em $H_{0}^{1}(\Omega)$. Portanto,

$$
q \beta\left\|u_{n}\right\|^{2} \leq 4 \pi p, \text { para todo } n \geq n_{0}, \text { com } p<\frac{1}{1-\|v\|^{2}}
$$

Usando o Lema 3.10, existe $C_{2}>0$, tal que para todo $n \geq n_{0}$,

$$
\begin{aligned}
\int_{\Omega}\left|f\left(x, u_{n}\right)\right|^{q} d x & \leq C \int_{\Omega} e^{q \beta u_{n}^{2}} d x=C \int_{\Omega} e^{q \beta\left\|u_{n}\right\|^{2} v_{n}^{2}} d x \\
& \leq C \int_{\Omega} e^{4 \pi p v_{n}^{2}} d x \leq C_{2}
\end{aligned}
$$

o que verifica a Afirmação 3.

Usando (3.12), para cada $v=u_{n}-u \operatorname{com} n \geq n_{0}$, temos

$$
\left|\int_{\Omega} \nabla u_{n} \nabla\left(u_{n}-u\right) d x-\int_{\Omega} f\left(x, u_{n}\right)\left(u_{n}-u\right) d x\right| \leq \epsilon_{n}\left\|u_{n}-u\right\|
$$

Pela desigualdade de Hölder e da Afirmação 3, para todo $n \geq n_{0}$, tem-se

$$
\begin{aligned}
\mid \int_{\Omega} \nabla u_{n} \nabla\left(u_{n}-u\right) d x & \leq \int_{\Omega}\left|f\left(x, u_{n}\right)\left(u_{n}-u\right)\right| d x+\epsilon_{n}\left\|u_{n}-u\right\| \\
& \leq\left(\int_{\Omega}\left|f\left(x, u_{n}\right)\right|^{q} d x\right)^{1 / p}\left(\int_{\Omega}\left|u_{n}-u\right|^{q^{\prime}} d x\right)^{1 / q^{\prime}}+\epsilon_{n}\left\|u_{n}-u\right\| \\
& \leq C_{2}^{1 / p}\left\|u_{n}-u\right\|_{L^{q^{\prime}}}+\epsilon_{n}\left\|u_{n}-u\right\| .
\end{aligned}
$$

Como $\left\{\left\|u_{n}-u\right\|\right\}$ é uma sequência limitada , $\epsilon_{n} \rightarrow 0$ e $u_{n} \rightarrow u$ em $L^{p}(\Omega)$, para todo $p \geq$ 1 , então

$$
\lim _{n \rightarrow \infty} \int_{\Omega} \nabla u_{n} \nabla\left(u_{n}-u\right) d x=0
$$


Logo,

$$
\left\|u_{n}-u\right\|^{2}=\int_{\Omega} \nabla\left(u_{n}-u\right) \nabla\left(u_{n}-u\right) d x=\int_{\Omega} \nabla\left(u_{n}-u\right) \nabla u_{n} d x-\int_{\Omega} \nabla\left(u_{n}-u\right) \nabla u d x .
$$

De $u_{n} \rightarrow u$ em $H_{0}^{1}(\Omega)$ e de (3.23), temos

$$
\lim _{n \rightarrow \infty} u_{n}=u, \quad \text { em } H_{0}^{1}(\Omega)
$$

Usando (3.19), temos que $\phi(u)=c$, o qual é uma contradição com o suposto e, portanto, $0 \leq \phi(u)<c$ não é possível.

Corolário 3.12. Suponha (H1), (H2) e (H3), então

a) Se f possui crescimento subcrítico, então $\phi$ satisfaz a condição $(P S)_{c}$ para cada $c \in \mathbb{R}$.

b) Se $f$ possui crescimento crítico, então $\phi$ satisfaz a condição $(P S)_{c}$ para cada $c \in$ $\left(-\infty, \frac{2 \pi}{\alpha_{0}}\right)$

\section{Demonstração.}

a) Dado $c \in \mathbb{R}$, escolha $\beta>0$ tal que $c<\frac{2 \pi}{\beta}$, logo, pela Afirmação 3.6, (3.3) é satisfeito. Portanto, pela Proposição 3.11 se satisfaz a condição $(P S)_{c}$.

b) Dado $c<\frac{2 \pi}{\alpha_{0}}$, escolha $\beta>0$ tal que $c<\frac{2 \pi}{\beta}<\frac{2 \pi}{\alpha_{0}}$, como $\beta>\alpha_{0}$, pela Afirmação $3.6,(3.3)$ é satisfeito. Portanto, pela Proposição 3.11 se satisfaz a condição $(P S)_{c}$

Proposição 3.13. Assuma (H1) e (H2), Seja $Z$ um subespaço de dimensão finita em $H_{0}^{1}(\Omega)$. Então $\phi$ é limitada superiormente em $Z$, além disso, dado $M>0$ existe $R>0$ tal que

$$
\phi(u) \leq-M, \quad \text { para todo } u \in Z, \operatorname{com}\|u\| \geq R
$$

\section{Demonstração.}

Seja $u_{0} \in Z$, tal que $\left\|u_{0}\right\|=1$, defina a função

$$
\xi(t)=\phi\left(t u_{0}\right)=\frac{t^{2}}{2}-\int_{\Omega} F\left(x, t u_{0}\right) d x, \quad t \in \mathbb{R} .
$$


Pela parte $(b)$ do Lema $3.9 \operatorname{com} \epsilon=\frac{1}{p}$ e $p>2$, existe $t_{\epsilon}>0$, tal que

$$
0<p F(x, t) \leq f(x, t), \quad \forall x \in \Omega, \forall|t| \geq t_{\epsilon} .
$$

Isto é, a condição de Ambrosetti-Rabinowitz é satisfeita por $f$. Portanto, existem $C_{1}, C_{2}>0$, tais que

$$
F(x, t) \geq C_{1}|t|^{p}-C_{2}, \quad \forall x \in \Omega, \forall t \in \mathbb{R}
$$

Substituindo em (3.24), obtemos

$$
\begin{aligned}
\xi(t) & =\frac{t^{2}}{2}-\int_{\Omega} F\left(x, t u_{0}\right) d x \leq \frac{t^{2}}{2}-\int_{\Omega} C_{1}\left|t u_{0}\right|^{p}-C_{2} d x \\
& =\frac{t^{2}}{2}-C_{1}|t|^{p}\left\|u_{0}\right\|_{L^{P}}^{p} d x+C_{2}|\Omega| .
\end{aligned}
$$

Como $Z$ é um espaço de dimensão finita, todas as nomas em $Z$ são equivalentes , então existe $a>0$, tal que

$$
a\|u\| \leq\|u\|_{L^{p}}, \quad \forall u \in Z .
$$

Então isto e observando que $\left\|u_{0}\right\|=1$, segue que

$$
\xi(t)=\frac{t^{2}}{2}-C_{1}|t|^{p}\left\|u_{0}\right\|_{L^{P}}^{p} d x+C_{2}|\Omega| \leq \frac{t^{2}}{2}-C_{1} a^{p}|t|^{p}+C_{2}|\Omega|
$$

E, portanto, $\xi$ é limitada superiormente e $\lim _{|t| \rightarrow+\infty} \xi(t)=-\infty$, isto é, existem $K, R>0$, tais que

$$
\xi(t) \leq K, \forall t \in \mathbb{R} \quad \text { e } \quad \xi(t) \leq-M, \forall|t| \geq R .
$$

Note que, $K$ e $R$ não dependem de $u_{0}$. Por conseguinte, para todo $u \in Z \backslash 0$, temos $u=t u_{1} \operatorname{com} t \in \mathbb{R}$ e $\left\|u_{1}\right\|=1, \log \mathrm{O}$

$$
\phi(u)=\phi\left(t u_{1}\right)=\xi(t) \leq K
$$

Isto é, $\phi$ é limitado superiormente em $Z$.

Por outro lado, se $u \in Z \operatorname{com}\|u\| \geq R$, temos que $u=t u_{1}$ com $\left\|u_{1}\right\|=1$ e $|t| \geq R$ e, portanto,

$$
\phi(u)=\phi\left(t u_{1}\right)=\xi(t) \leq-M
$$

Proposição 3.14. Suponha (H1), (H2), (H4) e a condição (3.3). Então existem $a>0$ e $\rho>0$, 
tais que

$$
\phi(u) \geq a, \quad \text { se }\|u\|=\rho .
$$

Demonstração. De (H4), podemos escolher $\mu>0$, tal que

$$
\limsup _{t \rightarrow 0} \frac{2 F(x, t)}{t^{2}}<\mu<\lambda_{1}, \quad \text { uniformemente em } x \in \Omega .
$$

Então existe $\delta>0$, tal que

$$
F(x, t) \leq \frac{\mu t^{2}}{2}, \quad \text { se }|t|<\delta .
$$

Logo, da parte (c) do Lema 3.9 e a condição (3.3)

$$
F(x, t) \leq \hat{M}|f(x, t)| \leq C \hat{M} e^{\beta t^{2}}, \quad \forall x \in \Omega, \forall t \in \mathbb{R} .
$$

Observe que se $|t| \geq \delta$, então $1 \leq \frac{|t|^{q}}{\delta^{q}}$ e, portanto,

$$
F(x, t) \leq \frac{C \hat{M}}{\delta^{q}} e^{\beta t^{2}}|t|^{q}=C_{1} e^{\beta t^{2}}|t|^{q}, \quad \forall x \in \Omega, \forall|t| \geq \delta .
$$

Logo, de (3.25) e (3.26), obtemos

$$
F(x, t) \leq C_{1} e^{\beta t^{2}}|t|^{q}+\frac{\mu t^{2}}{2}, \quad \forall x \in \Omega, \forall t \in \mathbb{R} .
$$

Pela definição de $\lambda_{1}$, (3.27) e a desigualdade de Hölder, obtemos

$$
\begin{aligned}
\phi(u) & =\frac{1}{2}\|u\|^{2} \int_{\Omega} F(x, u) d x \geq \frac{1}{2}\|u\|^{2}-\int_{\Omega} C_{1} e^{\beta u^{2}}|u|^{q}+\frac{\mu|u|^{2}}{2} d x \\
& \geq \frac{1}{2}\|u\|^{2}-\frac{\mu}{2}\|u\|_{L^{2}}^{2}-C_{1}\left(\int_{\Omega} e^{p \beta u^{2}} d x\right)^{1 / p}\left(\int_{\Omega}|u|^{p^{\prime} q} d x\right)^{1 / p^{\prime}} \\
& \geq \frac{1}{2}\left(1-\frac{u}{\lambda_{1}}\right)\|u\|^{2}-C_{1}\|u\|_{L^{p q^{\prime}}}^{q}\left(\int_{\Omega} e^{p \beta u^{2}} d x\right)^{1 / p} .
\end{aligned}
$$

Seja $0<\delta_{0}$, tal que $\beta p \delta_{0}^{2}<4 \pi$, pelo Teorema 2.9, existe $C>0$ tal que

$$
\left(\int_{\Omega} e^{p \beta u^{2}} d x\right)^{1 / p}=\left(\int_{\Omega} e^{p \beta\|u\|^{2}\left(\frac{u}{\|u\|}\right)^{2}} d x\right)^{1 / p} \leq C \quad \text { se }\|u\| \leq \delta_{0} .
$$

Usando também a imersão $L^{q^{\prime} p}(\Omega) \hookrightarrow H_{0}^{1}(\Omega)$ em (3.28), existe $C_{2}>0$ tal que 


$$
\phi(u) \geq \frac{1}{2}\left(1-\frac{u}{\lambda_{1}}\right)\|u\|^{2}-C_{2}\|u\|^{q}, \quad \text { se }\|u\| \leq \delta_{0} .
$$

Escolhendo $0<\rho \leq \min \left\{\delta_{0},\left(\frac{1}{4 C_{2}}\left(1-\frac{\mu}{\lambda_{1}}\right)\right)^{1 / q-2}\right\}$ e $a=\frac{1}{2}\left(1-\frac{u}{\lambda_{1}}\right) \rho^{2}$, temos

$$
\phi(u) \geq a, \quad \text { se }\|u\|=\rho .
$$

Denote por $V$ o subespaço de $H_{0}^{1}(\Omega)$ gerado pelas autofunções $\varphi_{j}$ de $\left(-\Delta, H_{0}^{1}(\Omega)\right)$, correspondentes aos autovalores $\lambda_{j}$, para $j=1, \ldots, k$. Denote também $W=V^{\perp}$.

Observe que $0<\lambda_{1}<\lambda_{2} \leq \lambda_{3} \leq \ldots$ e $\varphi_{j}$ são ortogonais em $L^{2}(\Omega)$ e $H_{0}^{1}(\Omega)$.

Observação 3.15. Usando as notações como acima. Tem-se

a) Se $u \in V$, então $\|u\|^{2} \leq \lambda_{k}\|u\|_{L^{2}}^{2}$.

b) Se $u \in W$, então $\|u\|^{2} \lambda_{k+1} \geq\|u\|_{L^{2}}^{2}$.

\section{Demonstração.}

a) Seja $u \in V$, então $u=\sum_{j=1}^{k} \alpha_{j} \varphi_{j} \operatorname{com} \alpha_{j} \in \mathbb{R}, j=1, \ldots, k$, logo, usando o fato de ortogonalidade e o fato de que $\left\{\lambda_{j}\right\}_{j \geq 1}$ são crescentes, obtemos

$$
\begin{aligned}
\|u\|^{2} & =\sum_{j=1}^{k} \alpha_{j}^{2}\left\|\varphi_{j}\right\|^{2}=\sum_{j=1}^{k} \alpha_{j}^{2} \int_{\Omega}\left|\nabla \varphi_{j}\right|^{2} d x=\sum_{j=1}^{k} \alpha_{j}^{2} \lambda_{j} \int_{\Omega}\left|\varphi_{j}\right|^{2} d x . \\
& \leq \lambda_{k} \sum_{j=1}^{k} \alpha_{j}^{2} \int_{\Omega}\left|\varphi_{j}\right|^{2} d x=\lambda_{k} \sum_{j=1}^{k} \alpha_{j}^{2}\left\|\varphi_{j}\right\|_{L^{2}}^{2}=\lambda_{k}\|u\|_{L^{2}}^{2} .
\end{aligned}
$$

b) Seja $u \in W$, então $u=\sum_{j=k+1}^{\infty} \alpha_{j} \varphi_{j}, \operatorname{com} \alpha_{j} \in \mathbb{R}, j \geq k+1, \log$,

$$
\begin{aligned}
\|u\|^{2} & =\sum_{j=k+1}^{\infty} \alpha_{j}^{2}\left\|\varphi_{j}\right\|^{2}=\sum_{j=k+1}^{\infty} \alpha_{j}^{2} \int_{\Omega}\left|\nabla \varphi_{j}\right|^{2} d x=\sum_{j=k+1}^{\infty} \alpha_{j}^{2} \lambda_{j} \int_{\Omega}\left|\varphi_{j}\right|^{2} d x \\
& \geq \lambda_{k+1} \sum_{j=k+1}^{\infty} \alpha_{j}^{2} \int_{\Omega}\left|\varphi_{j}\right|^{2} d x=\lambda_{k+1} \sum_{j=k+1}^{\infty} \alpha_{j}^{2}\left\|\varphi_{j}\right\|_{L^{2}}^{2}=\lambda_{k+1}\|u\|_{L^{2}}^{2} .
\end{aligned}
$$


Proposição 3.16. Assuma (H1), (H2), (H5) e a condição (3.3). Então existem $a>0$ e $\rho>0$, tais que

$$
\phi(u) \geq a, \text { se }\|u\|=\rho .
$$

Demonstração. Pelo (H5) $F(x, t) \leq \frac{1}{2} \mu t^{2}$, se $|t| \leq \delta$, com $\mu<\lambda_{k+1}$. Logo, usando a parte $(b)$ da Observação 3.15 e procedendo como na Proposição 3.14, temos que existe $\delta_{0}>0$, tal que

$$
\phi(u) \geq \frac{1}{2}\left(1-\frac{\mu}{\lambda_{k+1}}\right)\|u\|^{2}-C_{2}\|u\|^{q}, \quad \text { se } u \in W, \operatorname{com}\|u\| \leq \delta_{0} .
$$

Logo, existem $\rho>0$ e $a>0$, tais que

$$
\phi(u) \geq a, \quad \text { se } u \in W, \operatorname{com}\|u\| \leq \delta_{0} .
$$

\subsection{Demonstrações dos Teoremas}

\subsubsection{O caso subcrítico}

Demonstração do Teorema 3.2. Para provar a existência de solução usaremos o Teorema do Passo da Montanha (ver Apêndice A, Teorema A.13). Para isso verifiquemos as hipóteses do teorema. Seja $X=H_{0}^{1}(\Omega)$, pela Proposição $3.8 \phi \in \mathcal{C}^{1}\left(H_{0}^{1}(\Omega), \mathbb{R}\right)$, pelo Corolário $3.12 \phi$ satisfaz a condição $(P S)_{c}$ para todo $c \in \mathbb{R}$, da definição de $\phi$, temos $\phi(0)=0$. Além disso, vejamos que se satisfaz as condições $i$ ) e $i i$ ) do Teorema A.13

i) É satisfeito pela Proposição 3.14.

ii) Seja $0 \neq u_{0} \in H_{0}^{1}(\Omega)$ e considere $Z=\left\{t u_{0}: t \in \mathbb{R}\right\}$, então $\operatorname{dim}(Z)<+\infty$, pela Proposição 3.13, existe $e \in Z$, tal que $\phi(e) \leq 0$ com $\|e\|>\rho$.

Então pelo Teorema A.13, $\phi$ possui um ponto crítico $c \geq a>0$. Consequentemente, o problema possui uma solução não trivial.

Para provar que o problema tem infinitas soluções verificaremos as hipóteses do Teorema A.14 do Apêndice A.

Sejam $V=0$ e $W=H_{0}^{1}(\Omega)$, pela hipótese $f(x, t)$ é uma função ímpar em $t$, então $F(x, t)=\int_{0}^{t} f(x, s) d s$ é uma função par em $t, \log$,

$$
\phi(-u)=\frac{1}{2}\|-u\|^{2}-\int_{\Omega} F(x,-u) d s=\phi(u) .
$$


Isto é, $\phi$ é uma função par. Além disso, se satisfazem as condições $i^{\prime}$ ) e $i i^{\prime}$ ) pois :

i') É satisfeito pela proposição 3.14 .

ii') É satisfeito pela proposição 3.13 .

Demonstração do Teorema 3.3. Para provar a existência de solução usaremos o Teorema do Passo da Montanha generalizado (ver Apêndice A, Teorema A.15). Considere $X=H_{0}^{1}(\Omega)$, o espaço $V$ gerado por as autofunções $\varphi_{j} \operatorname{com} j=1, \ldots, k$ e $W=V^{\perp}$, pela Proposição 3.8, $\phi \in \mathcal{C}^{1}\left(H_{0}^{1}(\Omega), \mathbb{R}\right)$, pelo Corolário $3.12 \phi$ satisfaz a condição $(P S)_{c}$ para todo $c \in \mathbb{R}$. Além disso, se satisfazem as condições $\left.i\right)$ e $\left.i i\right)$ do Teorema A.14, pois :

i) É satisfeito pela Proposição 3.14 .

ii) Seja $e=\varphi_{k+1}$, onde $\varphi_{k+1}$ é o autovalor normalizado associado ao autovalor $\lambda_{k+1}$, deste modo $\|e\|=1$, considerando o subespaço $Z=V \oplus V_{\lambda_{k+1}}$, onde $V_{\lambda_{k+1}}$ é o subespaço gerado por $\varphi_{k+1}$, então $\operatorname{dim}(Z)<+\infty$, pela Proposição 3.13, existe $R_{1}>0$, tal que $\phi(u) \leq 0$, se $u \in Z$ com $\|u\| \geq R_{1}$. Tome $R>\max \left\{R_{1}, \rho\right\}, \log$ o

$$
\phi(u) \leq 0, \quad \text { para todo } u \in Z, \text { com }\|u\| \geq R,
$$

e considerando

$$
Q=\left(\overline{B_{R}} \cap V\right) \oplus\left\{r \varphi_{k+1}: 0<r<R\right\}
$$

Vejamos que $\phi(u) \leq 0$, para todo $u \in \partial Q$.

Se $u \in \partial Q$, tem-se os seguintes casos:

a) $u=v+R \varphi_{k+1}, v \in \overline{B_{R}} \cap V$.

b) $u=v, v \in \overline{B_{R}} \cap V$.

c) $u=v+r \varphi_{k+1},\|v\|=R, 0<r<R$.

Caso a: Como $\|u\|^{2}=\|v\|^{2}+\left\|R \varphi_{k+1}\right\|^{2}=\|v\|^{2}+R^{2}$, então $\|u\| \geq R$, além disso $u \in Z$, logo, de (3.29), $\phi(u) \leq 0$.

Caso b: Note que $u \in V$, então pela parte $a$ ) da Observação 3.15, temos $\|u\|^{2} \leq$ $\lambda_{k}\|u\|_{L^{p}}^{2}$. Da condição (H6), temos $F(x, t) \geq \frac{1}{2} \lambda_{k} t^{2}, \forall x \in \Omega$, para todo $t \in \mathbb{R}$. Logo,

$$
\phi(u)=\frac{1}{2}\|u\|^{2}-\int_{\Omega} F(x, u) d x \leq \frac{1}{2}\|u\|^{2}-\frac{\lambda_{k}}{2}\|u\|_{L^{p}}^{2} \leq 0
$$


Caso c: Note que, $\|u\| \geq R$, então por (3.29), $\phi(u) \leq 0$.

Consequentemente, $\phi(u) \leq 0$, para todo $u \in \partial Q$.

Logo, pelo Teorema A.15, existe um valor crítico $c \geq a>0$, e por conseguinte, o problema possui uma solução não trivial.

Para provar que o problema tem infinitas soluções, basta verificar as hipóteses do Teorema A.14, de maneira análoga como foi feita no Teorema 3.2.

\subsubsection{O caso crítico}

Pela parte $b$ ) do Corolário 3.12, a condição $(P S)_{c}$ no caso crítico só é satisfeita se $c<\frac{2 \pi}{\alpha_{0}}$, onde $\alpha_{0}$ é o índice de criticalidade. O seguinte Lema mostra que o nível minimax do passo da montanha é menor que $\frac{2 \pi}{\alpha_{0}}$.

Lema 3.17. Com as hipóteses do Teorema 3.4, existe $\omega \in H_{0}^{1}(\Omega)$ com $\|\omega\|=1$, tal que

$$
\max \{\phi(t \omega): t \geq 0\}<\frac{2 \pi}{\alpha_{0}}
$$

Demonstração. Começamos definindo as seguintes funções

$$
\bar{\omega}_{n}(x)= \begin{cases}\sqrt{\ln n}, & 0 \leq|x|<\frac{1}{n} \\ \ln \frac{1}{|x|}, & \frac{1}{n} \leq|x|<1 \\ \frac{\sqrt{\ln n}}{n} & 1 \leq|x| .\end{cases}
$$

Então

$$
\nabla \bar{\omega}_{n}(x)=\frac{1}{\sqrt{2 \pi}} \begin{cases}0, & 0 \leq|x|<\frac{1}{n} \text { ou }|x| \geq 1 \\ -\frac{x}{|x|^{2} \sqrt{\ln n}}, & \frac{1}{n} \leq|x|<1\end{cases}
$$

Note que,

$$
\left\|\bar{\omega}_{n}\right\|_{B_{1}(0)}^{2}=\int_{B_{1}(0)}\left|\nabla \bar{\omega}_{n}(x)\right|^{2} d x=\frac{1}{2 \pi \ln n} \int_{0}^{2 \pi} \int_{\frac{1}{n}}^{1} \frac{r}{r^{2}} d r=1 .
$$


Por conseguinte, $\bar{\omega}_{n} \in H_{0}^{1}\left(B_{1}(0)\right)$ e $\left\|\bar{\omega}_{n}\right\|=1$, para todo $n \geq 1$. Como $d$ é o raio interno de $\Omega$, existe $x_{0} \in \Omega$, tal que $B_{d}\left(x_{0}\right) \subset \Omega$. Defina

$$
\omega_{n}=\bar{\omega}_{n}\left(\frac{x-x_{0}}{d}\right), \quad \text { para cada } n \geq 1 .
$$

Então

$$
\omega_{n} \in H_{0}^{1}(\Omega), \quad \operatorname{supp}\left(\omega_{n}\right) \subset B_{d}\left(x_{0}\right), \text { para cada } n \geq 1 .
$$

Afirmação. Existe $n_{0} \in \mathbb{N}$, tal que

$$
\max \left\{\phi\left(t \omega_{n_{0}}\right): t \geq 0\right\}<\frac{2 \pi}{\alpha_{0}} .
$$

De fato, suponha por contradição, que isso não ocorre, ou seja

$$
\max \left\{\phi\left(t \omega_{n}\right): t \geq 0\right\} \geq \frac{2 \pi}{\alpha_{0}}, \quad \text { para todo } n \geq 1
$$

Pelas Proposições 3.13 e 3.14, para cada $n \geq 1$ existe $t_{n}>0$, tal que

$$
\phi\left(t_{n} \omega_{n}\right)=\max \left\{\phi\left(t \omega_{n}\right): t \geq 0\right\}
$$

ou seja

$$
\phi\left(t_{n} \omega_{n}\right)=\frac{t_{n}^{2}}{2}\left\|w_{n}\right\|-\int_{\Omega} F\left(x, t_{n} w_{n}\right) \geq \frac{2 \pi}{\alpha_{0}}, \quad \text { para todo } n \geq 1 .
$$

Como $\left\|w_{n}\right\|=1$, para cada $n \geq 1$ e da condição (H3) a integral é positiva e, portanto,

$$
t_{n}^{2} \geq \frac{2 \pi}{\alpha_{0}}, \quad \text { para todo } n \geq 1
$$

Por outro lado, de (3.30), para todo $n \geq 1$, temos

$$
\frac{d \phi\left(t \omega_{n}\right)}{d t}=0, \quad \text { em } t=t_{n} .
$$

Então,

$$
t_{n}-\int_{\Omega} f\left(x, t_{n} \omega_{n}\right) \omega_{n} d x=0
$$

Logo,

$$
t_{n}^{2}=\int_{\Omega} f\left(x, t_{n} \omega_{n}\right) t_{n} \omega_{n} d x \geq \int_{B_{\frac{d}{n}}\left(x_{0}\right)} f\left(x, t_{n} \omega_{n}\right) t_{n} \omega_{n} d x, \quad \text { para todo } n \geq 1 .
$$


Dado $\epsilon>0$, de (H7), existe $s_{\epsilon}>0$, tal que

$$
f(x, s) s \geq(\beta-\epsilon) e^{\alpha_{0} s^{2}}, \quad \text { para cada } s \geq s_{\epsilon}
$$

Como $\omega_{n}(x)=\sqrt{\ln n}$, para cada $x \in B_{\frac{d}{n}}\left(x_{0}\right)$ e $\left\{t_{n}\right\}$ é uma sequência limitada inferiormente, então existe $n_{1} \in \mathbb{N}$, tal que

$$
t_{n} \omega_{n}(x) \geq s_{\epsilon}, \quad \text { em } \quad B_{\frac{d}{n}}\left(x_{0}\right), \quad \text { se } n \geq n_{1},
$$

logo, de (3.32), (3.33) e usando a definição de $\omega_{n}$, temos para cada $n \geq n_{1}$,

$$
\begin{aligned}
t_{n}^{2} & \geq(\beta-\epsilon) \int_{B_{\frac{d}{n}}\left(x_{0}\right)} e^{\alpha_{0} t_{n}^{2} \omega_{n}^{2}(x)} d x=(\beta-\epsilon) e^{\alpha_{0} t_{n}^{2} \frac{\ln n}{2 \pi}} \int_{B_{\frac{d}{n}}\left(x_{0}\right)} d x \\
& =(\beta-\epsilon) e^{\alpha_{0} t_{n}^{2} \frac{\ln n}{2 \pi} \pi} \frac{d^{2}}{n^{2}}=(\beta-\epsilon) \pi d^{2} e^{2 \ln n\left(\frac{\alpha_{0} t_{n}^{2}}{4 \pi}-1\right)} .
\end{aligned}
$$

Suponha que exista uma subsequência $\left\{t_{k_{n}}\right\}$ de $\left\{t_{n}\right\}$, tal que $\lim _{n \rightarrow \infty} t_{k_{n}}=+\infty$, então usando (3.34),

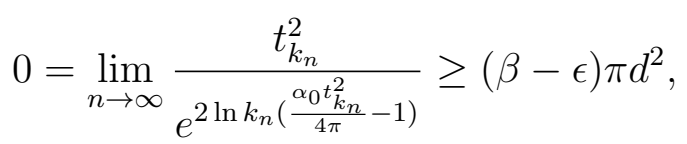

donde temos uma contradição e, portanto, $\left\{t_{n}\right\}$ é uma sequência limitada.

Por outro lado, suponha que $\lim _{n \rightarrow \infty} t_{n}^{2} \neq \frac{4 \pi}{\alpha_{0}}$, como $t_{n}^{2} \geq \frac{4 \pi}{\alpha_{0}}$, então existe uma subsequência $\left\{t_{k_{n}}\right\}$ de $\left\{t_{n}\right\}$ tal que $\lim _{n \rightarrow \infty} t_{k_{n}}^{2}=t_{0}^{2}>\frac{4 \pi}{\alpha_{0}}$, logo como em (3.35), temos uma contradição, por conseguinte,

$$
\lim _{n \rightarrow \infty} t_{n}^{2}=\frac{4 \pi}{\alpha_{0}}
$$

Defina, para cada $n \geq 1$,

$$
A_{n}=\left\{x \in B_{d}\left(x_{0}\right): t_{n} \omega_{n}(x) \geq s_{\epsilon}\right\}, \quad B_{n}=B_{d}\left(x_{0}\right) \backslash A_{n} .
$$

Provemos a seguinte afirmação

Afirmação. Com as definições acima, tem-se as seguintes convergências.

$$
t_{n} \omega_{n}(x) \rightarrow 0 \text {, q.t.p. em } B_{d}\left(x_{0}\right) \quad \text { e } \quad \chi_{B_{n}} \rightarrow 1 \text {, q.t.p. em } B_{d}\left(x_{0}\right)
$$

De fato, seja $x \in B_{d}\left(x_{0}\right) \backslash\left\{x_{0}\right\}$, existe $n_{0} \in \mathbb{N}$, tal que $\frac{d}{n_{0}}<\left|x-x_{0}\right|$ deste modo, 
$x \in B_{d}\left(x_{0}\right) \backslash B_{\frac{d}{n}}\left(x_{0}\right)$, para todo $n \geq n_{0}$. Logo,

$$
t_{n} \omega_{n}(x)=\frac{t_{n} \ln \frac{d}{\left|x-x_{0}\right|}}{\sqrt{\ln n}} \leq C_{2} \frac{\ln n_{0}}{\ln n}, \quad \text { para todo } n \geq n_{0}
$$

onde $C_{2}>0$ é uma cota superior de $\left\{t_{n}\right\}$. E, portanto,

$$
t_{n} \omega_{n}(x) \rightarrow 0 \text {, q.t.p. em } B_{d}\left(x_{0}\right)
$$

Logo, para $x \in B_{d}\left(x_{0}\right) \backslash\left\{x_{0}\right\}$, existe $n_{1} \in \mathbb{N}$, tal que $t_{n} \omega_{n}(x)<s_{\epsilon}$ para cada $n \geq n_{1}$, ou seja, $\chi_{B_{n}}=1$, para cada $n \geq n_{1}$. Assim

$$
\chi_{B_{n}} \rightarrow 1 \text {, q.t.p. em } B_{d}\left(x_{0}\right) .
$$

Como em (3.32), temos

$$
\begin{aligned}
t_{n}^{2} & \geq \int_{B_{d}\left(x_{0}\right)} f\left(x, t_{n} \omega_{n}\right) t_{n} \omega_{n} d x=\int_{B_{n}} f\left(x, t_{n} \omega_{n}\right) t_{n} \omega_{n} d x+\int_{B_{d}\left(x_{0}\right) \backslash B_{n}} f\left(x, t_{n} \omega_{n}\right) t_{n} \omega_{n} d x \\
& \geq \int_{B_{n}} f\left(x, t_{n} \omega_{n}\right) t_{n} \omega_{n} d x+(\beta-\epsilon) \int_{B_{d}\left(x_{0}\right) \backslash B_{n}} e^{\alpha_{0} t_{n}^{2} w_{n}^{2}} d x \\
& =\int_{B_{n}} f\left(x, t_{n} \omega_{n}\right) t_{n} \omega_{n} d x+(\beta-\epsilon) \int_{B_{d}\left(x_{0}\right)} e^{\alpha_{0} t_{n}^{2} \omega_{n}^{2}} d x-(\beta-\epsilon) \int_{B_{n}} e^{\alpha_{0} t_{n}^{2} \omega_{n}^{2}} d x .
\end{aligned}
$$

Analisaremos cada integral por separadamente . Note que,

$$
\int_{B_{n}}\left|f\left(x, t_{n} \omega_{n}\right) t_{n} \omega_{n}\right| d x \leq C_{3} \int_{B_{n}}\left|t_{n} \omega_{n}\right| d x=C_{3} \int_{B_{d}\left(x_{0}\right)}\left|t_{n} \omega_{n}\right| \chi_{B_{n}} d x
$$

onde $C_{3}=\sup _{(x, s) \in \bar{\Omega} \times\left[0, s_{\epsilon}\right]}|f(x, s)|$. Logo, por (3.36), temos

$$
\left|t_{n} \omega_{n}\right| \chi_{B_{n}} \rightarrow 0, \quad \text { q.t.p. em } B_{d}\left(x_{0}\right)
$$

Além disso,

$$
\left|t_{n} \omega_{n} \chi_{B_{n}}\right| \leq s_{\epsilon}, \quad \operatorname{com} s_{\epsilon} \in L^{2}\left(B_{d}\left(x_{0}\right)\right) .
$$

Pelo Teorema da Convergência Dominada de Lebesque, temos

$$
\int_{B_{n}} f\left(x, t_{n} \omega_{n}\right) t_{n} \omega_{n} d x \rightarrow 0, \quad \text { se } n \rightarrow \infty
$$


Fazendo a mudança de variável $y=\frac{x-x_{0}}{d}$ e do fato que $t_{n}^{2} \geq \frac{4 \pi}{\alpha_{0}}$, temos

$$
\begin{aligned}
\int_{B_{d}\left(x_{0}\right)} e^{\alpha_{0} t_{n}^{2} \omega_{n}^{2}(x)} d x & \geq \int_{B_{d}\left(x_{0}\right)} e^{4 \pi \omega_{n}^{2}(x)} d x=d^{2} \int_{B_{1}(0)} e^{4 \pi \bar{\omega}_{n}^{2}(y)} d y \\
& =d^{2} \int_{0}^{2 \pi}\left\{\int_{0}^{\frac{1}{n}} e^{4 \pi \frac{\ln n}{2 \pi}} r d r+\int_{\frac{1}{n}}^{1} e^{4 \pi \frac{\left(\ln \frac{1}{r}\right)^{2}}{2 \pi \ln n}} r d r\right\} d \theta \\
& =2 \pi d^{2}\left(\frac{1}{2}+\int_{\frac{1}{n}}^{1} e^{2 \frac{\left(\ln \frac{1}{r}\right)^{2}}{\ln n}} r d r\right)
\end{aligned}
$$

Fazendo na última integral a mudança de variável, $s=\frac{\left(\ln \frac{1}{r}\right)^{2}}{\ln n}$, obtemos

$$
\int_{B_{d}\left(x_{0}\right)} e^{\alpha_{0} t_{n}^{2} \omega_{n}^{2}(x)} d x \geq 2 \pi d^{2}\left(\frac{1}{2}+\int_{\frac{1}{n}}^{1} e^{2 \frac{\left(\ln \frac{1}{r}\right)^{2}}{\ln n}} r d r\right)=2 \pi d^{2}\left(\frac{1}{2}+\frac{2 \ln n \int_{0}^{1} e^{2 \ln n\left(s^{2}-s\right)} d s}{2}\right)
$$

Do Lema B.5, temos $\lim _{m \rightarrow \infty} m \int_{0}^{1} e^{m\left(s^{2}-s\right)} d s=2$, então tomando limite em (3.38), obtemos

$$
\lim _{n \rightarrow \infty} \int_{B_{d}\left(x_{0}\right)} e^{\alpha_{0} t_{n}^{2} \omega_{n}^{2}(x)} d x \geq 3 \pi d^{2} .
$$

Agora,

$$
\int_{B_{n}} e^{\alpha_{0} t_{n}^{2} \omega_{n}^{2}} d x=\int_{B_{d}\left(x_{0}\right)} e^{\alpha_{0} t_{n}^{2} \omega_{n}^{2}} \chi_{B_{n}} d x
$$

Por (3.36), temos

$$
e^{\alpha_{0} t_{n}^{2} \omega_{n}^{2}} \chi_{B_{n}} \rightarrow 1, \quad \text { q.t.p. em } B_{d}\left(x_{0}\right)
$$

Além disso,

$$
\left|e^{\alpha_{0} t_{n}^{2} \omega_{n}^{2}} \chi_{B_{n}}\right| \leq e^{\alpha_{0} s_{\epsilon}^{2}}, \quad \operatorname{com} e^{\alpha_{0} s_{\epsilon}^{2}} \in L^{2}\left(B_{d}\left(x_{0}\right)\right) .
$$

Pelo Teorema da Convergência Cominada de Lebesque, temos

$$
\int_{B_{d}\left(x_{0}\right)} e^{\alpha_{0} t_{n}^{2} \omega_{n}^{2}(x)} d x \rightarrow \int_{B_{d}\left(x_{0}\right)} 1 d x=\pi d^{2}, \quad \text { se } n \rightarrow \infty
$$

Fazendo $n \rightarrow \infty$ em (3.37), encontramos

$\lim _{n \rightarrow \infty} t_{n}^{2} \geq \lim _{n \rightarrow \infty} \int_{B_{n}} f\left(x, t_{n} \omega_{n}\right) t_{n} \omega_{n} d x+(\beta-\epsilon) \lim _{n \rightarrow \infty} \int_{B_{d}\left(x_{0}\right)} e^{\alpha_{0} t_{n}^{2} \omega_{n}^{2}} d x-(\beta-\epsilon) \lim _{n \rightarrow \infty} \int_{B_{n}} e^{\alpha_{0} t_{n}^{2} \omega_{n}^{2}} d x$,

donde

$$
\frac{4 \pi}{\alpha_{0}} \geq 2 \pi(\beta-\epsilon) d^{2}
$$


Como $\epsilon>0$ é arbitrário, tem-se que $\beta \leq \frac{2}{\alpha_{0} d^{2}}$, o qual é uma contradição.

Demonstração do Teorema 3.4.

Para provar a existência de solução verificaremos que se satisfazem hipóteses do Teorema A.13.

Seja $X=H_{0}^{1}(\Omega)$, pela proposição $3.15, \phi \in \mathcal{C}^{1}\left(H_{0}^{1}(\Omega, \mathbb{R})\right)$. Vejamos que se satisfazem as condições $i$ ) e $i i$ )

i) Pela Proposição 3.14, existem $a>0$ e $\rho>0$, tal que

$$
\phi(u) \geq a, \quad \text { se }\|u\|=\rho, u \in W .
$$

ii) Pelo Lema 3.17, existe $\omega_{0} \in H_{0}^{1}(\Omega)$, tal que

$$
\max \left\{\phi\left(t \omega_{0}\right): t \geq 0\right\}<\frac{2 \pi}{\alpha_{0}} .
$$

Pela Proposição 3.13, com $Z=\left\{t \omega_{0}: t \in \mathbb{R}\right\}$ existe $R>0$, tal que para todo $u \in Z$ e $\|u\| \geq R$, tem-se $\phi(u) \leq 0$, logo, tomando $t_{0}>\rho$, e considerando $e=t_{0} \omega_{0} \in H_{0}^{1}(\Omega)$, como $\left\|\omega_{0}\right\|=1$, temos $\|e\|>\rho$ e $e \in Z$ assim, $\phi(e) \leq 0$.

Resta verificar que $\phi$ satisfaz a condição $(P S)_{c}, \operatorname{com} c=\inf _{\gamma \in \Gamma} \sup _{t \in[0,1]} \phi(\gamma(t))$, onde

$$
\Gamma=\left\{\gamma \in \mathcal{C}\left([0,1], H_{0}^{1}(\Omega)\right): \gamma(0)=0 \text { e } \gamma(1)=e\right\}
$$

De fato, defina

$$
\begin{aligned}
\gamma_{0}:[0,1] & \rightarrow H_{0}^{1}(\Omega) \\
t & \mapsto \gamma_{0}(t)=t t_{0} \omega_{0}
\end{aligned}
$$

então $\gamma_{0} \in \Gamma$, além disso

$$
c=\inf _{\gamma \in \Gamma} \sup _{t \in[0,1]} \phi(\gamma(t)) \leq \sup _{t \in[0,1]} \phi\left(\gamma_{0}(t)\right)=\sup _{t \in[0,1]} \phi\left(t t_{0} \omega_{0}\right) \leq \sup _{t \geq 0} \phi\left(t \omega_{0}\right)<\frac{2 \pi}{\alpha_{0}} .
$$

Logo, pelo Corolário 3.12, a condição $(P S)_{c}$ é satisfeita. Então, pelo Teorema A.13, $\phi$ possui um ponto crítico $c \geq a>0$. E, portanto, o problema possui uma solução não trivial. 


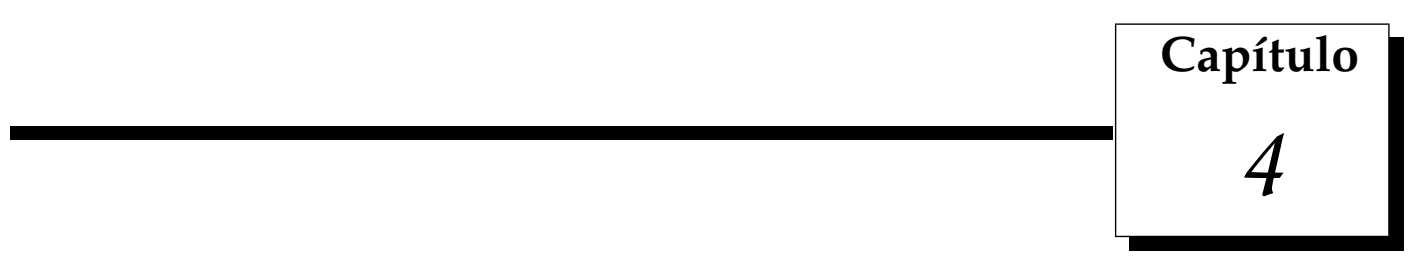

\section{Existência e não existência de soluções radiais}

Neste capítulo estudaremos o problema

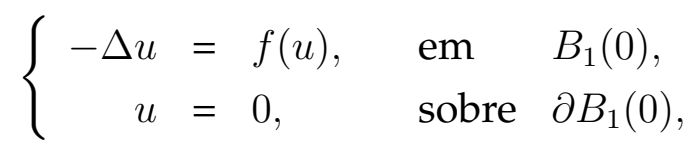

onde $B_{1}(0)$ é a bola unitária em $\mathbb{R}^{2}$ e a função $f$ possui crescimento crítico exponencial da forma $f(r)=e^{\alpha_{0} r^{2}} h(r)$, para algum $\alpha_{0}>0$ e $h \in \mathcal{C}(\mathbb{R})$ possui crescimento subcrítico, ou seja

$$
\lim _{|r| \rightarrow+\infty} \frac{|h(r)|}{e^{\alpha r^{2}}}=0, \quad \text { para todo } \alpha>0,
$$

ou equivalentemente, $\lim _{|r| \rightarrow+\infty} \frac{\ln |h(r)|}{r^{2}}=0$. Deste modo podemos escrever a equação (4.1), no caso onde $f$ possui crescimento crítico como:

$$
\left\{\begin{aligned}
-\Delta u & =\operatorname{sgn}(h(u)) e^{\alpha_{0} u^{2}+\ln |h(u)|} & & \text { em } \quad \Omega, \\
u & =0 & & \text { sobre } \partial \Omega,
\end{aligned}\right.
$$

O interesse desde capítulo é determinar a existência e a não existência do problema (4.1) em termos de $h$. Este capítulo é baseada no artigo [8] de De Figueiredo e Ruf. 


\subsection{Formulação do problema}

Consideramos somente soluções radiais e positivas do seguinte problema

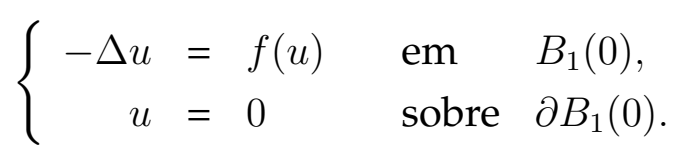

Sendo $u$ uma função radial podemos escrever $u\left(x_{1}, x_{2}\right)=U(r)$, para todo $\left(x_{1}, x_{2}\right) \in$ $B_{1}(0)$, onde $r=\sqrt{x_{1}^{2}+x_{2}^{2}}$. Logo

$$
\frac{\partial}{\partial x_{i}} u=U^{\prime}(r) \frac{\partial}{\partial x_{i}} r=U^{\prime}(r) \frac{x_{i}}{r}, \text { para } i=1,2
$$

$\mathrm{e}$

$$
\frac{\partial^{2}}{\partial x_{i}^{2}} u=U^{\prime \prime}(r) \frac{x_{i}^{2}}{r^{2}}+U^{\prime}(r)\left(\frac{1}{r}-\frac{x_{i}^{2}}{r^{3}}\right), \text { para } i=1,2 .
$$

E, portanto,

$$
-\nabla u=-\left(\frac{\partial^{2}}{\partial x_{1}^{2}} u+\frac{\partial^{2}}{\partial x_{2}^{2}} u\right)=U^{\prime \prime}(r)+\frac{1}{r} U^{\prime}(r) .
$$

Também, como $u=0$ em $\partial B_{1}(0)$, temos $U(1)=0$. Além disso, como $U$ é radial, temos $U(h)=u(h, 0)=u(-h, 0)$, para todo $h \in \mathbb{R}$. Logo,

$$
U^{\prime}(0)=\lim _{h \rightarrow 0} \frac{U(h)-U(0)}{h}=\lim _{h \rightarrow 0} \frac{u(h, 0)-u(0,0)}{h}=\frac{\partial}{\partial x_{1}} u(0,0) .
$$

Por outro lado,

$$
U^{\prime}(0)=\lim _{h \rightarrow 0} \frac{U(h)-U(0)}{h}=\lim _{h \rightarrow 0} \frac{u(-h, 0)-u(0,0)}{h}=-\frac{\partial}{\partial x_{1}} u(0,0) .
$$

Donde concluímos que $U^{\prime}(0)=0$. Deste modo, reduzimos a equação (4.1) para a equação radial

$$
\begin{gathered}
U^{\prime \prime}(r)+\frac{1}{r} U^{\prime}(r)+f(U)=0, \quad 0<r<1 \\
U(1)=0, U^{\prime}(0)=0 .
\end{gathered}
$$

Para encontrar soluções do problema (4.4), usamos o método de "shotting", ou seja consideraremos para cada $\gamma>0$ o seguinte problema de valor inicial

$$
\begin{gathered}
U^{\prime \prime}(r)+\frac{1}{r} U^{\prime}(r)+f(U)=0, \quad 0<r<1, \\
U(0)=\gamma, U^{\prime}(0)=0 .
\end{gathered}
$$


Denotaremos por $U_{\gamma}$, a solução do problema (4.5) e por $R(\gamma)$ o primeiro zero positivo tal que $U_{\gamma}(R(\gamma))=0$. Logo, se existe $\gamma_{0}>0$ tal que $R\left(\gamma_{0}\right)=1$, então $U_{\gamma_{0}}$ será uma solução do problema (4.4), por outro lado, se $R(\gamma) \neq 1, \forall \gamma>0$, então o problema (4.1) não tem solução, pois não existe uma solução que satisfaça a condição $U(1)=0$. Deste modo, encontrar soluções do problema (4.3) se reduz a estudar o comportamento de $R(\gamma)$. Fazendo as mudanças de variáveis $t=-2 \ln \left(\frac{r}{2}\right), y(t)=U(r)$ e considerando o caso que $f$ seja uma função positiva convertemos a equação (4.5), em

$$
\begin{aligned}
-y^{\prime \prime} & =f(y) e^{-t}=e^{g(y)-t}, \quad t>2 \ln 2 \\
y(+\infty) & =\gamma, y^{\prime}(+\infty)=0 .
\end{aligned}
$$

Note que, como $f$ possui crescimento crítico tem-se: $f(r)=e^{\alpha_{0} r^{2}+\ln h(r)}$, desse modo, $g(r)=\alpha_{0} r^{2}+\ln h(r)$, mostraremos depois que podemos supor que $\alpha_{0}=1$. Assim é suficiente estudar o problema

$$
\begin{aligned}
-y^{\prime \prime} & =e^{y^{2}+\ln h(y)-t}, \quad t>2 \ln 2 \\
y(+\infty) & =\gamma, y^{\prime}(+\infty)=0 .
\end{aligned}
$$

Com $h$ satisfazendo as seguintes hipóteses:

- $h: \mathbb{R} \rightarrow \mathbb{R}$ é de classe $\mathcal{C}^{2}$, e suponha que existem $r_{1}>0$ e $\sigma>0$ tais que para certas constantes $K>0$ e $c>0$.

$$
\begin{aligned}
& \left(A_{1}\right) h(r)=\frac{K}{r^{a}}, \text { para } r \geq r_{1}, a>0 \\
& \left(A_{2}\right) 0 \leq h(r) \leq c K r^{1+\sigma}, \text { para } 0 \leq r \leq r_{1} .
\end{aligned}
$$

Mostraremos que a solubilidade de (4.7) depende do parâmetro $a>0$ em $h$. Para provar os resultados, analisaremos a dependência de $\lim _{\gamma \rightarrow+\infty} T(\gamma)$ sobre o exponente $a>0$ em $h$, onde $T(\gamma)$ é o primeiro zero (desde $+\infty$ ) da solução $y_{\gamma}$ de (4.7). Observe que para calcular o limite somente precisamos considerar valores de $\gamma$ suficientemente grandes.

\subsection{Estimativas}

Nesta seção estimaremos o comportamento das soluções da equação (4.6), com $g(U)=\ln f(U)$ da forma $g(U)=\alpha U^{2}+\ln h(U)$. Note que, fazendo a mudança $y(t)=\sqrt{\alpha} U(t)$ na equação (4.6), temos a equação $-y^{\prime \prime}(t)=\sqrt{\alpha} h\left(\frac{y}{\sqrt{\alpha}}\right) e^{y^{2}-t}$. Desde 
que $h\left(\frac{r}{\sqrt{\alpha}}\right)=\alpha^{a / 2} \frac{K}{r^{a}}$, para $r \geq \sqrt{\alpha} r_{1}$, podemos restringir a funções $g(U)$ da forma $g(U)=U^{2}+\ln h(U)$, com a correspondente mudança de $K$ a $\alpha^{(1+a) / 2} K$ em os termos de $h$ e, portanto, é suficiente considerar a equação (4.7).

Lema 4.1. Dado qualquer numero $\gamma>0$, existe exatamente uma solução $U_{\gamma}$ de (4.5). Além disso, se $R(\gamma)$ denota o primeiro valor de $r$ tal que $U_{\gamma}(r)=0$; então $R(\gamma)$ depende continuamente de $\gamma$.

Demonstração. (Veja [3]).

Denotamos por $T_{1}=T_{1}(\gamma)$ o valor tal que $U_{\gamma}\left(T_{1}\right)=r_{1}$.

Lema 4.2. Suponha que $g \in \mathcal{C}^{2}\left(\left[r_{1},+\infty\right)\right), g^{\prime}(t)>0$ e $g^{\prime \prime}(t) \geq 0$ em $\left[r_{1},+\infty\right)$, então para cada $T_{1} \leq t<+\infty$, tem-se

(i) $y(t, \gamma) \leq \gamma-\frac{2}{g^{\prime}(\gamma)} \ln \left(1+\frac{1}{2} g^{\prime}(\gamma) e^{g(\gamma)-t}\right)$.

(ii) $g(y(t, \gamma)) \geq g(\gamma)-2 \ln \left(1+\frac{1}{2} g^{\prime}(\gamma) e^{g(\gamma)-t}\right)$.

Demonstração. Denotemos por $y(t)$ (ou $y$ ) a $y(t, \gamma)$. Usando a equação (4.6), temos

$$
-\int_{t}^{+\infty} y^{\prime \prime}(s) d s=\int_{t}^{+\infty} e^{g(y(s))-s} d s
$$

Logo, como $y^{\prime}(+\infty)=0$, obtemos

$$
y^{\prime}(t)=\int_{t}^{+\infty} e^{g(y(s))-s} d s
$$

Note que, da hipótese e da equação anterior que $g$ e $y$ têm derivadas positivas, portanto $g(y(\cdot))$ é uma função crescente. Logo

$$
\int_{t}^{+\infty} e^{g(y(t))-s} d s \leq \int_{t}^{+\infty} e^{g(y(s))-s} d s \leq \int_{t}^{+\infty} e^{g(y(+\infty))-s}, d s
$$

Assim,

$$
e^{g(y(t))} \int_{t}^{+\infty} e^{-s} d s \leq y^{\prime}(s) \leq e^{g(\gamma)} \int_{t}^{+\infty} e^{-s} d s
$$

Logo,

$$
e^{g(y(t))-t} \leq y^{\prime}(t) \leq e^{g(\gamma)-t}
$$

Logo, tomando logaritmo e somando $t$, temos

$$
g(y(t)) \leq \ln y^{\prime}(t)+t=\leq g(\gamma)
$$


Como $g$ é uma função contínua e $\lim _{t \rightarrow+\infty} y(t)=\gamma$, resulta

$$
\lim _{t \rightarrow+\infty} \ln y^{\prime}(t)+t=g(\gamma)
$$

Por outro lado, defina

$$
E(t)=y^{\prime}(t)-\frac{1}{2} y^{\prime}(t)^{2} g^{\prime}(y(t))-e^{g(y(t))-t} .
$$

Usando (4.6), temos

$$
E^{\prime}(t)=-\frac{1}{2} y^{\prime}(t)^{3} g^{\prime \prime}(y(t))
$$

Como $y^{\prime \prime} \geq 0$ e $g^{\prime \prime} \geq 0$, então $E^{\prime} \leq 0$ isto é, $E$ é uma função decrescente.

Além disso, temos

$$
\begin{aligned}
\lim _{t \rightarrow+\infty} E(t) & =y^{\prime}(+\infty)-\frac{1}{2} y^{\prime}(+\infty)^{2} g^{\prime}(y(+\infty))-e^{g(y(+\infty))} \lim _{t \rightarrow+\infty} e^{-t} \\
& =0-\frac{1}{2}(0)^{2} g^{\prime}(\gamma)-e^{g(\gamma)}(0)=0
\end{aligned}
$$

e

$$
0 \leq \frac{E(t)}{y^{\prime}(t)}=1-\frac{1}{2} y^{\prime}(t) g^{\prime}(y(t))-\frac{e^{g(y(t))-t}}{y^{\prime}(t)} .
$$

Como $-y^{\prime \prime}(t)=e^{g(y(t))-t}$, então

$$
0 \leq 1-\frac{1}{2} y^{\prime}(t) g^{\prime}(y(t))+\frac{y^{\prime \prime}(t)}{y^{\prime}(t)}=\frac{E(t)}{y^{\prime}(t)}
$$

Usando (4.10), temos $E(t)=-\int_{t}^{+\infty} E^{\prime}(s) d s$, logo de (4.9),

$$
0 \leq 1-\frac{1}{2} y^{\prime}(t) g^{\prime}(y(t))+\frac{y^{\prime \prime}(t)}{y^{\prime}(t)}=\frac{1}{2 y^{\prime}(t)} \int_{t}^{+\infty} y^{\prime}(s)^{3} g^{\prime \prime}(y(s)) d s
$$

Note que, como $y^{\prime \prime} \leq 0, y^{\prime}$ é decrescente; logo

$$
\begin{aligned}
\int_{t}^{+\infty} y^{\prime}(s)^{3} g^{\prime \prime}(y(s)) d s & \leq y^{\prime}(t)^{2} \int_{t}^{+\infty} g^{\prime \prime}(y(s)) y^{\prime}(s) d s \\
& =y^{\prime}(t)^{2}\left(g^{\prime}(\gamma)-g^{\prime}(y(t))\right) .
\end{aligned}
$$

Substituindo a desigualdade acima em (4.11), temos

$$
0 \leq 1-\frac{1}{2} y^{\prime}(t) g^{\prime}(y(t))+\frac{y^{\prime \prime}(t)}{y^{\prime}(t)} \leq \frac{1}{2} y^{\prime}(t)\left(g^{\prime}(\gamma)-g^{\prime}(y(t))\right)
$$


Integrando a primeira desigualdade de (4.12), obtemos

$$
\begin{aligned}
0 \leq \int_{t}^{+\infty}\left(1-\frac{1}{2} y^{\prime}(s) g^{\prime}(y(s))+\frac{y^{\prime \prime}(s)}{y^{\prime}(s)}\right) d s= & \int_{t}^{+\infty} \frac{d}{d s}\left(s-\frac{g(y(s))}{2}+\ln y^{\prime}(s)\right) d s \\
= & s-\frac{g(y(s))}{2}+\left.\ln y^{\prime}(s)\right|_{t} ^{+\infty} \\
= & \lim _{s \rightarrow+\infty}\left(s+\ln y^{\prime}(s)-\frac{g(y(s))}{2}\right)+\frac{g(y(t))}{2} \\
& -t-\ln y^{\prime}(t) \\
= & \frac{g(\gamma)+g(y(t))}{2}-t-\ln y^{\prime}(t) .
\end{aligned}
$$

Logo,

$$
\ln y^{\prime}(t) \leq \frac{g(\gamma)+g(y(t))}{2}-t
$$

E, portanto,

$$
y^{\prime}(t) \leq e^{\frac{g(\gamma)+g(y(t))}{2}-t}
$$

Assim,

$$
y^{\prime}(t) e^{-\frac{g(y(t))}{2}} \leq e^{\frac{g(\gamma)}{2}-t} .
$$

Sendo $g^{\prime}$ e $y$ funções crescentes, temos que $g^{\prime}(y(t)) \leq g^{\prime}(\gamma)$, multiplicando isto na desigualdade acima, tem-se

$$
g^{\prime}(y(t)) y^{\prime}(t) e^{-\frac{g(y(t))}{2}} \leq g^{\prime}(\gamma) e^{\frac{g(\gamma)}{2}-t}
$$

Integrando

$$
-2 \int_{t}^{+\infty} \frac{d}{d s}\left(e^{-\frac{g(y(s))}{2}}\right) d s \leq-g^{\prime}(\gamma) \int_{t}^{+\infty} \frac{d}{d s}\left(e^{\frac{g(\gamma)}{2}-s}\right) d s .
$$

Logo,

$$
-2 \lim _{s \rightarrow+\infty} e^{-\frac{g(y(s))}{2}}+2 e^{-\frac{g(y(t))}{2}} \leq-g^{\prime}(\gamma)\left(\lim _{s \rightarrow \infty} e^{\frac{g(\gamma)}{2}-s}-e^{\frac{g(\gamma)}{2}-t}\right) .
$$

Assim,

$$
e^{-\frac{g(y(t))}{2}}-e^{-\frac{g(\gamma)}{2}} \leq \frac{1}{2} g^{\prime}(\gamma) e^{\frac{g(\gamma)}{2}-t}
$$

Multiplicando por $e^{\frac{g(\gamma)}{2}}$,

$$
e^{-\frac{g(\gamma)-g(y(t))}{2}}-1 \leq \frac{1}{2} g^{\prime}(\gamma) e^{g(\gamma)-t}
$$

Logo,

$$
\frac{g(\gamma)-g(y(t))}{2} \leq \ln \left(1+\frac{1}{2} g^{\prime}(\gamma) e^{g(\gamma)-t}\right)
$$


Donde temos a desigualdade $(i i)$ :

$$
g(\gamma)-2 \ln \left(1+\frac{1}{2} g^{\prime}(\gamma) e^{g(\gamma)-t}\right) \leq g(y(t))
$$

Agora, integrando as duas últimas desigualdades de (4.12), obtemos

$$
\int_{t}^{+\infty}\left(1-\frac{1}{2} y^{\prime}(s) g^{\prime}(y(s))+\frac{y^{\prime \prime}(s)}{y^{\prime}(s)}\right) d s \leq \frac{1}{2} \int_{t}^{+\infty} y^{\prime}(s)\left(g^{\prime}(\gamma)-g^{\prime}(y(s))\right) d s .
$$

Então

$$
\begin{aligned}
\frac{g(\gamma)+g(y(t))}{2}-t-\ln y^{\prime}(t) & \leq\left.\frac{1}{2}\left(g^{\prime}(\gamma) y(s)-g(y(s))\right)\right|_{t} ^{+\infty} \\
& =\frac{1}{2}\left(\gamma g^{\prime}(\gamma)-g(\gamma)+g(y(t))-y(t) g^{\prime}(\gamma)\right)
\end{aligned}
$$

Simplificando

$$
g(\gamma)-t \leq \ln y^{\prime}(t)+\frac{1}{2}(\gamma-y(t)) g^{\prime}(\gamma)
$$

Assim,

$$
e^{g(\gamma)-t} \leq y^{\prime}(t) e^{\frac{(\gamma-y(t)) g^{\prime}(\gamma)}{2}}
$$

Integrando

$$
-\int_{t}^{+\infty} \frac{d}{d s}\left(e^{g(\gamma)-s}\right) \leq-\frac{2}{g^{\prime}(\gamma)} \int_{t}^{+\infty} \frac{d}{d s}\left(e^{\frac{(\gamma-y(s)) g^{\prime}(\gamma)}{2}}\right) .
$$

Donde,

$$
e^{g(\gamma)-t} \leq \frac{2}{g^{\prime}(\gamma)}\left(e^{\frac{(\gamma-y(t)) g^{\prime}(\gamma)}{2}}-1\right)
$$

Logo,

$$
1+\frac{g^{\prime}(\gamma)}{2} e^{g(\gamma)-t} \leq e^{\frac{(\gamma-y(t)) g^{\prime}(\gamma)}{2}}
$$

E, portanto,

$$
\ln \left(1+\frac{g^{\prime}(\gamma)}{2} e^{g(\gamma)-t}\right) \leq \frac{(\gamma-y(t)) g^{\prime}(\gamma)}{2} .
$$

Finalmente obtemos a desigualdade $(i)$ :

$$
y(t) \leq \gamma-\frac{2}{g^{\prime}(\gamma)} \ln \left(1+\frac{g^{\prime}(\gamma)}{2} e^{g(\gamma)-t}\right) .
$$

Note que, a função $g(r)=r^{2}+\ln \left(\frac{K}{r^{a}}\right) \operatorname{com} K$ e $a$ números positivos satisfazem as condições do Lema 4.2. no intervalo $[\sqrt{a / 2},+\infty)$. 
Definimos $T_{5}=g(\gamma)-4 \ln \left(\frac{g^{\prime}(\gamma)}{2}\right)$ e consideramos a função $G(t)=1+\frac{g^{\prime}(\gamma)}{2} e^{g(\gamma)-t}$.

Lema 4.3. Suponha que $g(r)=r^{2}+\ln \left(\frac{K}{r^{a}}\right)$, com $a>0$, para $r \geq r_{1}$. Então a solução de (4.6) satisfaz para $t \geq T_{5}$

$$
g(\gamma)-2 \ln G(t) \leq g(y(t)) \leq g(\gamma)-2 \ln G(t)+\frac{\ln ^{2} G(t)}{\gamma^{2}}+O\left(\frac{\ln G(t)}{\gamma^{3}}\right)
$$

Demonstração. Como y é uma função crescente para $t \geq T_{5} \geq T_{1}$, temos $y(t) \geq y\left(T_{1}\right)=$ $r_{1}$. Aplicando $g^{-1}$ na desigualdade $(i i)$ do Lema 4.2 e observando que $g^{-1}$ é uma função crescente, temos

$$
y(t) \geq g^{-1}(g(\gamma)-2 \ln G(t))
$$

Usando o Polinômio de Taylor do segundo grau, obtemos

$$
g^{-1}\left(b_{1}+b_{2}\right)=g^{-1}\left(b_{1}\right)+b_{2}\left(g^{-1}\right)^{\prime}\left(b_{1}\right)+b_{2}^{2} \frac{1}{2}\left(g^{-1}\right)^{\prime \prime}(\tilde{g}),
$$

com $b_{1}+b_{2}<\tilde{g}<b_{1}$, onde $b_{1}=g(\gamma)$ e $b_{2}=-2 \ln G(t)$. Assim, existe $\tilde{g}$ com $g(\gamma)-2 \ln G(t)<\tilde{g}<g(\gamma)$ e

$$
y(t) \geq \gamma-2 \ln G(t)\left(g^{-1}\right)^{\prime}(g(\gamma))+2 \ln ^{2} G(t)\left(g^{-1}\right)^{\prime \prime}(\tilde{g}) .
$$

Sendo $g$ uma função contínua crescente existe um único valor $\tilde{\gamma}$, tal que $\tilde{g}=g(\tilde{\gamma})$. Como $g(\tilde{\gamma})=\tilde{g}<g(\gamma)$ e $g$ função crescente então $\tilde{\gamma}<\gamma$, além disso, como $G(t)$ é uma função decrescente, temos

$$
g(\gamma)-2 \ln G(t) \leq g(\gamma)-2 \ln G\left(T_{5}\right) \leq g(\tilde{\gamma})
$$

Agora, faremos uma estimativa da segunda derivada de $g^{-1}$ em $\tilde{g}$ a qual é dada por

$$
\left(g^{-1}\right)^{\prime \prime}(\tilde{g})=-\frac{g^{\prime \prime}(\tilde{\gamma})}{g^{\prime}(\gamma)^{3}}
$$

A partir das expressões de $g^{\prime}$ e $g$, obtemos

$$
g^{\prime}(r) \geq \frac{2}{r} g(r), \text { se } r \geq e^{1 / 2} K^{1 / a} .
$$

Note que, como estamos supondo $\gamma$ grande, temos $\tilde{\gamma}$ é grande, logo, usando (4.14) e $\gamma>\tilde{\gamma}$, obtemos

$$
g^{\prime}(\tilde{\gamma}) \geq \frac{2}{\tilde{\gamma}} g(\tilde{\gamma}) \geq \frac{2}{\gamma}(g(\gamma)-2 \ln G(t))
$$


Por outro lado, como $G$ é uma função decrescente, temos $G(t) \leq G\left(T_{5}\right)$ se $T_{5} \leq t$, logo para $\gamma$ suficientemente grande, temos

$$
G(t) \leq G\left(T_{5}\right)=1+\frac{g^{\prime}(\gamma)}{2} e^{g(\gamma)-T_{5}}=1+\left(\frac{g^{\prime}(\gamma)}{2}\right)^{5}=1+\left(\gamma-\frac{a}{2 \gamma}\right)^{5}<\gamma^{6}
$$

Assim $\ln G(t) \leq 6 \ln \gamma$, usando isto em (4.16), obtemos

$$
\begin{aligned}
g^{\prime}(\tilde{\gamma}) & \geq \frac{2}{\gamma}\left(\gamma^{2}+\ln \left(\frac{K}{\gamma^{a}}\right)-12 \ln \gamma\right) \\
& =\frac{2}{\gamma}\left(\gamma^{2}+\ln K-a \ln \gamma-12 \ln \gamma\right) \\
& =2 \gamma-\frac{\ln \gamma}{\gamma}(24+2 a)+O\left(\frac{1}{\gamma}\right) \\
& =2 \gamma\left(1-\frac{\ln \gamma}{\gamma^{2}}(12+a)+O\left(\frac{1}{\gamma^{2}}\right)\right) .
\end{aligned}
$$

Usando o fato $(1-x)^{n} \geq 1-n x$, para $n \in \mathbb{N}$ e $x \in \mathbb{R}$, temos

$$
\begin{aligned}
g^{\prime}(\tilde{\gamma})^{3} & \geq 8 \gamma^{3}\left(1-\frac{\ln \gamma}{\gamma^{2}}(12+a)+O\left(\frac{1}{\gamma^{2}}\right)\right)^{3} \\
& \geq 8 \gamma^{3}\left(1-3 \frac{\ln \gamma}{\gamma^{2}}(12+a)+O\left(\frac{1}{\gamma^{2}}\right)\right) .
\end{aligned}
$$

Note que, $g^{\prime \prime}(\tilde{\gamma})=2+\frac{2}{\tilde{\gamma}^{2}}=2+O\left(\frac{1}{\gamma^{2}}\right)$ e, portanto,

$$
\frac{2+O\left(\frac{1}{\gamma^{2}}\right)}{8 \gamma^{3}\left(1-3 \frac{\ln \gamma}{\gamma^{2}}(12+a)+O\left(\frac{1}{\gamma^{2}}\right)\right)} \geq \frac{g^{\prime \prime}(\tilde{\gamma})}{g^{\prime}(\tilde{\gamma})^{3}} .
$$

Substituindo em (4.15), temos

$$
\left(g^{-1}\right)^{\prime \prime}(\tilde{g}) \geq-\frac{1+O\left(\frac{1}{\gamma^{2}}\right)}{4 \gamma^{3}\left(1-3 \frac{\ln \gamma}{\gamma^{2}}(12+a)+O\left(\frac{1}{\gamma^{2}}\right)\right)}
$$

Agora, usando o fato $\frac{1}{1-x}=1+x+x^{2}+\cdots$, para $x=3 \frac{\ln \gamma}{\gamma^{2}}(12+a)+O\left(\frac{1}{\gamma^{2}}\right)$ e notando 
que para as potências maiores que 2 tem ordem menor que $O\left(\frac{1}{\gamma^{2}}\right)$, resulta

$$
\left(g^{-1}\right)^{\prime \prime}(\tilde{g}) \geq-\frac{1+O\left(\frac{1}{\gamma^{2}}\right)}{4 \gamma^{3}}\left(1+3 \frac{\ln \gamma}{\gamma^{2}}(12+a)+O\left(\frac{1}{\gamma^{2}}\right)\right)
$$

Usando (4.17) em (4.13), temos

$$
\begin{aligned}
y(t) & \geq \gamma-\frac{2}{g^{\prime}(\gamma)} \ln G(t)-\frac{\ln ^{2} G(t)}{2 \gamma^{3}}\left(1+O\left(\frac{1}{\gamma^{2}}\right)\right)\left(1+3 \frac{\ln \gamma}{\gamma^{2}}(12+a)+O\left(\frac{1}{\gamma^{2}}\right)\right) \\
& =\gamma-\frac{2}{g^{\prime}(\gamma)} \ln G(t)-\frac{\ln ^{2} G(t)}{2 \gamma^{3}}-\frac{3 \ln \gamma}{2 \gamma^{5}}(12+a) \ln ^{2} G(t)+O\left(\frac{\ln ^{2} G(t)}{\gamma^{5}}\right) \\
& =\gamma-\frac{2}{g^{\prime}(\gamma)} \ln G(t)-\frac{1}{2 \gamma^{3}} \ln ^{2} G(t)+O\left(\frac{\ln ^{2} G(t)}{\gamma^{4}}\right) .
\end{aligned}
$$

Note também que

$$
\begin{aligned}
\frac{1}{g^{\prime}(\gamma)} & =\frac{1}{2 \gamma-\frac{a}{\gamma}}=\frac{1}{2 \gamma\left(1-\frac{a}{2 \gamma^{2}}\right)} \\
& =\frac{1}{2 \gamma}\left(1+\frac{a}{2 \gamma^{2}}+\left(\frac{a}{2 \gamma^{2}}\right)^{2}+\left(\frac{a}{2 \gamma^{2}}\right)^{3}+\cdots\right) \\
& =\frac{1}{2 \gamma}\left(1+\frac{a}{2 \gamma^{2}}\right)+O\left(\frac{1}{\gamma^{5}}\right) .
\end{aligned}
$$

Usando (4.19) em (4.26), temos

$$
\begin{aligned}
y(t) & \geq \gamma-2 \ln G(t)\left(\frac{1}{2 \gamma}\left(1+\frac{a}{2 \gamma^{2}}\right)+O\left(\frac{1}{\gamma^{5}}\right)\right)-\frac{1}{2 \gamma^{3}} \ln ^{2} G(t)+O\left(\frac{\ln ^{2} G(t)}{\gamma^{4}}\right) \\
& =\gamma-\frac{\ln G(t)}{\gamma}-\frac{a \ln G(t)}{2 \gamma^{3}}+O\left(\frac{\ln G(t)}{\gamma^{5}}\right)-\frac{1}{2 \gamma^{3}} \ln ^{2} G(t)+O\left(\frac{\ln ^{2} G(t)}{\gamma^{4}}\right) .
\end{aligned}
$$

Como

$$
O\left(\frac{\ln G(t)}{\gamma^{5}}\right)+O\left(\frac{\ln ^{2} G(t)}{\gamma^{4}}\right)=O\left(\frac{\ln ^{2} G(t)}{\gamma^{4}}\right)
$$

resulta

$$
y(t) \geq \gamma-\frac{\ln G(t)}{\gamma}-\frac{a \ln G(t)+\ln ^{2} G(t)}{2 \gamma^{3}}+O\left(\frac{\ln ^{2} G(t)}{\gamma^{4}}\right) .
$$


Por outro lado, pelo item $(i)$ do Lema 4.2 e por (4.19), temos

$$
\begin{aligned}
y(t) & \leq \gamma-2 \ln G(t)\left(\frac{1}{2 \gamma}\left(1+\frac{a}{2 \gamma^{2}}\right)+O\left(\frac{1}{\gamma^{5}}\right)\right) \\
& =\gamma-\frac{\ln G(t)}{\gamma}\left(1+\frac{a}{2 \gamma^{2}}\right)+O\left(\frac{\ln G(t)}{\gamma^{5}}\right) .
\end{aligned}
$$

Aplicando a função $g$, temos

$$
\begin{aligned}
g(y(t)) \leq & \left(\gamma-\frac{\ln G(t)}{\gamma}\left(1+\frac{a}{2 \gamma^{2}}\right)+O\left(\frac{\ln G(t)}{\gamma^{5}}\right)\right)^{2}+\ln K \\
& -a \ln \left(\gamma-\frac{\ln G(t)}{\gamma}\left(1+\frac{a}{2 \gamma^{2}}\right)+O\left(\frac{\ln G(t)}{\gamma^{5}}\right)\right) \\
= & \gamma^{2}+\frac{\ln ^{2} G(t)}{\gamma^{2}}\left(1+\frac{a}{2 \gamma^{2}}\right)^{2}-2 \ln G(t)\left(1+\frac{a}{2 \gamma^{2}}\right)+O\left(\frac{\ln G(t)}{\gamma^{4}}\right) \\
& +\ln K-a \ln \left(\gamma-\frac{\ln G(t)}{\gamma}\left(1+\frac{a}{2 \gamma^{2}}\right)+O\left(\frac{\ln G(t)}{\gamma^{5}}\right)\right) .
\end{aligned}
$$

Usando a identidade $\ln (x+z)=\ln x+\frac{z}{x}-\frac{1}{2}\left(\frac{z}{x}\right)^{2}+\frac{1}{3}\left(\frac{z}{x}\right)^{3}+\cdots$, com $x=\gamma \mathrm{e}$ $z=-\frac{\ln G(t)}{\gamma}\left(1+\frac{a}{2 \gamma^{2}}\right)+O\left(\frac{\ln G(t)}{\gamma^{5}}\right)$, obtemos

$$
\begin{aligned}
\ln \left(\gamma-\frac{\ln G(t)}{\gamma}\left(1+\frac{a}{2 \gamma^{2}}\right)+O\left(\frac{\ln G(t)}{\gamma^{5}}\right)\right) & =\ln \gamma-\frac{\ln G(t)}{\gamma^{2}}-\frac{a \ln G(t)}{4 \gamma^{4}}+\cdots \\
& =\ln \gamma-\frac{\ln G(t)}{\gamma^{2}}+O\left(\frac{\ln G(t)}{\gamma^{4}}\right) .
\end{aligned}
$$

Substituindo em (4.21), encontramos

$$
\begin{aligned}
g(y(t)) \leq & \gamma^{2}+\frac{\ln ^{2} G(t)}{\gamma^{2}}\left(1+\frac{a}{2 \gamma^{2}}\right)^{2}-2 \ln G(t)\left(1+\frac{a}{2 \gamma^{2}}\right)+O\left(\frac{\ln G(t)}{\gamma^{4}}\right) \\
& +\ln K-a \ln \gamma+a \frac{\ln G(t)}{\gamma^{2}}+O\left(\frac{\ln G(t)}{\gamma^{4}}\right) \\
= & \gamma^{2}+\ln K-a \ln \gamma-2 \ln G(t)+\frac{\ln ^{2} G(t)}{\gamma^{2}}+O\left(\frac{\ln G(t)}{\gamma^{4}}\right) \\
= & g(\gamma)-2 \ln G(t)+\frac{\ln ^{2} G(t)}{\gamma^{2}}+O\left(\frac{\ln G(t)}{\gamma^{4}}\right) .
\end{aligned}
$$

Portanto, usando a desigualdade acima e a parte (ii) do Lema 4.2 concluímos a prova do lema. 
Lema 4.4. Suponha que gé como no Lema 4.3. Então

$$
\begin{aligned}
\frac{2}{g^{\prime}(\gamma)} & =\frac{1}{\gamma}+\frac{1}{2 \gamma^{3}}+O\left(\frac{1}{\gamma^{4}}\right) \leq y^{\prime}(t) \leq \frac{1}{\gamma}+\frac{5}{2 \gamma^{3}}+O\left(\frac{\ln ^{2} \gamma}{\gamma^{4}}\right) \\
& =\frac{2}{g^{\prime}(\gamma)}+\frac{2}{\gamma^{3}}+O\left(\frac{\ln ^{2} \gamma}{\gamma^{4}}\right)
\end{aligned}
$$

para $T_{1} \leq t \leq T_{5}$, onde $T_{1}$ é tal que $y\left(T_{1}\right)=r_{1}$.

Demonstração. Usando o Lema 4.3, para $t \geq T_{5}$, temos

$$
\int_{t}^{+\infty} e^{g(\gamma)-2 \ln G(s)-s} d s \leq \int_{t}^{+\infty} e^{g(y(s))-s} d s \leq \int_{t}^{+\infty} e^{g(\gamma)-2 \ln G(s)-s+\frac{\ln ^{2} G(s)}{\gamma^{2}}+O\left(\frac{\ln G(s)}{\gamma^{3}}\right)} d s .
$$

Como $y^{\prime}(t)=\int_{t}^{+\infty} e^{g(y(s))-s} d s$, temos

$$
\int_{t}^{+\infty} e^{g(\gamma)-2 \ln G(s)-s} d s \leq y^{\prime}(t) \leq \int_{t}^{+\infty} e^{g(\gamma)-2 \ln G(s)-s+\frac{\ln ^{2} G(s)}{\gamma^{2}}+O\left(\frac{\ln G(s)}{\gamma^{3}}\right)} d s
$$

Fazendo $x=\frac{1}{2} g^{\prime}(\gamma) e^{g(\gamma)-s}$ na desigualdade , obtemos

$$
\frac{2}{g^{\prime}(\gamma)} \int_{0}^{\frac{1}{2} g(\gamma) e^{g(\gamma)-t}} \frac{1}{1+x^{2}} d x \leq y^{\prime}(t) \leq \frac{2}{g^{\prime}(\gamma)} \int_{0}^{\frac{1}{2} g(\gamma) e^{g(\gamma)-t}} \frac{e^{\frac{\ln ^{2}(1+x)}{\gamma^{2}}+O\left(\frac{\ln (1+x)}{\gamma^{3}}\right)}}{(1+x)^{2}} d x
$$

Agora para $t=T_{5}$ e fazendo a seguinte estimativa da exponencial

$$
\begin{aligned}
e^{\frac{\ln ^{2}(1+x)}{\gamma^{2}}+O\left(\frac{\ln (1+x)}{\gamma^{3}}\right)}= & 1+\frac{\ln ^{2}(1+x)}{\gamma^{2}}+O\left(\frac{\ln (1+x)}{\gamma^{3}}\right)+\frac{1}{2 !}\left(\frac{\ln ^{2}(1+x)}{\gamma^{2}}+O\left(\frac{\ln (1+x)}{\gamma^{3}}\right)\right)^{2} \\
& +\frac{1}{3 !}\left(\frac{\ln ^{2}(1+x)}{\gamma^{2}}+O\left(\frac{\ln (1+x)}{\gamma^{3}}\right)\right)^{3}+\cdots \\
= & 1+\frac{\ln ^{2}(1+x)}{\gamma^{2}}+O\left(\frac{\ln (1+x)}{\gamma^{3}}\right) \\
= & 1+\frac{\ln ^{2}(1+x)}{\gamma^{2}}+O\left(\frac{\ln (\gamma)}{\gamma^{3}}\right),
\end{aligned}
$$

no qual foi usado o fato $\ln G(t) \leq 6 \ln \gamma$, para $t=T_{5}$ e $\gamma$ suficientemente grande, assim $O\left(\frac{\ln (1+x)}{\gamma^{3}}\right)=O\left(\frac{\ln (\gamma)}{\gamma^{3}}\right)$. Logo, em (4.22), tem-se

$$
\frac{2}{g^{\prime}(\gamma)} \int_{0}^{\left(\frac{g^{\prime}(\gamma)}{2}\right)^{5}} \frac{1}{1+x^{2}} d x \leq y^{\prime}\left(T_{5}\right) \leq \frac{2}{g^{\prime}(\gamma)} \int_{0}^{\left(\frac{g^{\prime}(\gamma)}{2}\right)^{5}} \frac{1+O\left(\frac{\ln \gamma}{\gamma^{3}}\right)^{2}}{1+x}+\frac{\ln ^{2}(1+x)}{\gamma^{2}\left(1+x^{2}\right)} d x
$$


As integrais da desigualdade acima podem ser calculadas e estimadas para valores suficientemente grande de $z=\left(\frac{g^{\prime}(\gamma)}{2}\right)^{5}$. Assim temos:
a) $\int_{0}^{z} \frac{d x}{(1+x)^{2}}=\frac{z}{1+z}=1+O\left(\frac{1}{z}\right)$.
b) $\int_{0}^{z} \frac{\ln ^{2}(1+x)}{(1+x)^{2}} d x=\frac{2 z}{1+z}-\frac{2 \ln (1+z)}{1+z}-\frac{2 \ln ^{2}(1+z)}{1+z}=2+O\left(\frac{\ln ^{2} z}{z}\right)$.

Lembrando que $g^{\prime}(\gamma)=\gamma+O\left(\frac{1}{\gamma}\right)$ e usando as estimativas em (4.23), obtemos

$$
\frac{2}{g^{\prime}(\gamma)}\left(1+O\left(\frac{1}{\gamma^{5}}\right)\right) \leq y^{\prime}\left(T_{5}\right) \leq \frac{2}{g^{\prime}(\gamma)}\left(1+\frac{2}{\gamma^{2}}+O\left(\frac{\ln ^{2} \gamma}{\gamma^{3}}\right)\right)
$$

Agora estenderemos a estimativa para valores em $T_{1} \leq t \leq T_{5}$. Integrando (4.6) no intervalo $\left[t, T_{5}\right]$, obtemos

$$
y^{\prime}(t)=y^{\prime}\left(T_{5}\right)+\int_{t}^{T_{5}} e^{g(y(s))-s} d s
$$

Da parte $(i)$ do Lema 3.4, tem-se

$$
\ln \left(1+\frac{1}{2} g^{\prime}(\gamma) e^{g(\gamma)-t}\right) \leq \frac{1}{2} g^{\prime}(\gamma)(\gamma-y(t))
$$

Logo,

$$
\ln \left(\frac{1+\frac{1}{2} g^{\prime}(\gamma) e^{g(\gamma)-t}}{\frac{1}{e^{g(\gamma)-t}}+\frac{1}{2} g^{\prime}(\gamma)}\right) \leq \frac{1}{2} g^{\prime}(\gamma)(\gamma-y(t))-\ln \left(\frac{1}{e^{g(\gamma)-t}}+\frac{1}{2} g^{\prime}(\gamma)\right)
$$

E, portanto,

$$
g(y(t))-t \leq \frac{1}{2} g^{\prime}(\gamma)(\gamma-y(t))-\ln \left(\frac{1}{2} g^{\prime}(\gamma)\right)
$$

Defina

$$
\psi(y):=\frac{1}{2} g^{\prime}(\gamma)(\gamma-y(t))-\ln \left(\frac{1}{2} g^{\prime}(\gamma)\right) .
$$

Como $\psi^{\prime}(y)=g^{\prime \prime}(y) \geq 0$, temos que $\psi$ é uma função concava entre $y\left(T_{1}\right)$ e $y\left(T_{5}\right)$ e, portanto,

$$
\psi(t) \leq \max \left\{\psi\left(y\left(T_{1}\right)\right), \psi\left(y\left(T_{5}\right)\right)\right\}, \quad \text { para } T_{1} \leq t \leq T_{5} .
$$

Logo,

$$
g(y(t))-t \leq \max \left\{\psi\left(y\left(T_{1}\right)\right), \psi\left(y\left(T_{5}\right)\right)\right\}, \quad \text { para } T_{1} \leq t \leq T_{5}
$$


Note que,

$$
\begin{aligned}
\psi\left(y\left(T_{1}\right)\right) & =\psi\left(r_{1}\right)=g\left(r_{1}\right)-\frac{r_{1}}{2}\left(2 \gamma-\frac{a}{\gamma}\right)-\left(\gamma^{2}+\ln \left(\frac{K}{\gamma^{a}}\right)-\frac{\gamma}{2}\left(2 \gamma-\frac{a}{\gamma}\right)\right)-\ln \left(\frac{1}{2}\left(2 \gamma+\frac{a}{\gamma}\right)\right) \\
& =g\left(r_{1}\right)-\frac{r_{1}}{2}\left(2 \gamma-\frac{a}{\gamma}\right)-\ln \left(\frac{K}{\gamma^{a}}\right)-\frac{a}{2}-\ln \gamma+O\left(\frac{1}{\gamma^{2}}\right) .
\end{aligned}
$$

Por outro lado, pelo Lema 4.3, temos

$$
g(y(t))=g(\gamma)-2 \ln G(t)+O\left(\frac{\ln ^{2} G(t)}{\gamma^{2}}\right) .
$$

Também usando a parte $i$ ) do Lema 4.2 e (4.18), temos

$$
y(t)=\gamma-\frac{2}{g^{\prime}(\gamma)} \ln G(t)+O\left(\frac{\ln ^{2} G(t)}{\gamma^{2}}\right)
$$

Usando (4.28) e (4.29), resulta

$$
\begin{aligned}
\psi\left(y\left(T_{5}\right)\right)= & g\left(y\left(T_{5}\right)\right)-\frac{y\left(T_{5}\right)}{2}\left(2 \gamma-\frac{a}{\gamma}\right)-\left(\gamma^{2}+\ln \left(\frac{K}{\gamma^{a}}\right)-\frac{\gamma}{2}\left(2 \gamma-\frac{a}{\gamma}\right)\right)-\ln \left(\frac{1}{2}\left(2 \gamma+\frac{a}{\gamma}\right)\right) \\
= & g(\gamma)-2 \ln G\left(T_{5}\right)+O\left(\frac{\ln ^{2} G\left(T_{5}\right)}{\gamma^{2}}\right)-\frac{1}{2}\left(\gamma-\frac{2}{g^{\prime}(\gamma)} \ln G\left(T_{5}\right)\right. \\
& \left.+O\left(\frac{\ln ^{2} G\left(T_{5}\right)}{\gamma^{2}}\right)\right)\left(2 \gamma-\frac{a}{\gamma}\right)-\ln \left(\frac{K}{\gamma^{a}}\right)-\frac{a}{2}-\ln \gamma+O\left(\frac{1}{\gamma^{2}}\right) \\
= & -\ln \gamma-\ln \left(1+\left(\frac{g^{\prime}(\gamma)}{2}\right)^{5}\right)+O\left(\frac{\ln ^{2} \gamma}{\gamma^{2}}\right) .
\end{aligned}
$$

Portanto para $\gamma$ suficientemente grande, de (4.27) e (4.30), temos

$$
\psi\left(y\left(T_{1}\right)\right) \leq \psi\left(y\left(T_{5}\right)\right)
$$

Usando (4.26), obtemos

$$
\begin{aligned}
\int_{t}^{T_{5}} e^{g(y(s))-s} d s & \leq e^{\psi\left(y\left(T_{5}\right)\right)}\left(T_{5}-t\right)=e^{-\ln \gamma-\ln \left(1+\left(\frac{g^{\prime}(\gamma)}{2}\right)^{5}\right)+O\left(\frac{\ln ^{2} \gamma}{\gamma^{2}}\right)}\left(T_{5}-t\right) \\
& =e^{-\ln \left(\gamma\left(1+\frac{1}{2}\left(2 \gamma-\frac{a}{\gamma}\right)^{5}\right)\right)+O\left(\frac{\ln ^{2} \gamma}{\gamma^{2}}\right)}\left(T_{5}-t\right) \\
& =e^{-c \gamma^{6}}\left(T_{5}-t\right)=\frac{c}{\gamma^{6}}\left(T_{5}-t\right) \leq \frac{c}{\gamma^{6}}\left(T_{5}-T_{1}\right) .
\end{aligned}
$$


Pelo Teorema do valor médio, existe $\theta$ com $T_{1} \leq \theta \leq T_{5}$, e usando o fato que $y^{\prime}$ é decrescente, temos

$$
\frac{T_{5}-T_{1}}{y\left(T_{5}\right)-y\left(T_{1}\right)}=\frac{1}{y^{\prime}(\theta)} \leq \frac{1}{y^{\prime}\left(T_{5}\right)}
$$

Como $y\left(T_{1}\right) \geq 0$,

$$
T_{5}-T_{1} \leq \frac{y\left(T_{5}\right)-y\left(T_{1}\right)}{y^{\prime}\left(T_{5}\right)} \leq \frac{y\left(T_{5}\right)}{y^{\prime}\left(T_{5}\right)} \leq \frac{\gamma}{y^{\prime}\left(T_{5}\right)} .
$$

Usando a primeira desigualdade de (4.24), temos

$$
T_{5}-T_{1} \leq \frac{\gamma}{y^{\prime}\left(T_{5}\right)} \leq \frac{g^{\prime}(\gamma) \gamma}{2\left(1+O\left(\frac{1}{\gamma^{5}}\right)\right)}=\frac{2 \gamma^{2}-a}{2\left(1+O\left(\frac{1}{\gamma^{5}}\right)\right)}=O\left(\gamma^{2}\right)
$$

E, portanto, substituindo em (4.31) e logo usando (4.24), temos

$$
y^{\prime}\left(T_{5}\right) \leq y^{\prime}(t) \leq y^{\prime}\left(T_{5}\right)+O\left(\frac{1}{\gamma^{4}}\right), \quad \text { para } T_{1} \leq t \leq T_{5}
$$

Combinando a desigualdade anterior e (4.24), concluímos a prova do lema.

Lema 4.5. Seja g como no Lema 4.3. Então

$$
T_{1}=r_{1} \gamma+\left[\frac{1}{2}, \frac{5}{2}\right]-(a-1) \ln \gamma+\ln K+O\left(\frac{\ln ^{2} \gamma}{\gamma}\right)
$$

onde $[b, c]$ denota uma estimativa superior é inferior, isto é $w=[a, b] \Leftrightarrow a \leq w \leq b$.

Demonstração. Existe $\theta$ tal que $T_{1} \leq \theta \leq T_{5}$, satisfaz

$$
T_{1}=T_{5}-\frac{y\left(T_{5}\right)-y\left(T_{1}\right)}{y^{\prime}(\theta)} .
$$

Do Lema 4.4, temos a seguinte estimativa

$$
y^{\prime}(\theta)=\frac{1}{\gamma}\left(1+\frac{1}{\gamma^{2}}\left[\frac{1}{2}, \frac{5}{2}\right]+O\left(\frac{\ln ^{2} \gamma}{\gamma^{3}}\right)\right)
$$

Também

$$
\begin{aligned}
y^{\prime}\left(T_{5}\right) & =\gamma-\frac{1}{\gamma}\left(1+\frac{a}{2 \gamma^{2}}+O\left(\frac{1}{\gamma^{5}}\right)\right) \ln G\left(1+\left(\frac{g^{\prime}(\gamma)}{2}\right)^{5}\right)+O\left(\frac{\ln 1+\left(\frac{g^{\prime}(\gamma)}{2}\right)^{5}}{\gamma^{3}}\right) \\
& =\gamma-\frac{1}{\gamma} \ln \left(\frac{g^{\prime}(\gamma)}{2}\right)^{5}+O\left(\frac{\ln \left(\frac{g^{\prime}(\gamma)}{2}\right)^{5}}{\gamma^{3}}\right) .
\end{aligned}
$$


Substituindo em (4.32), temos

$$
\begin{aligned}
T_{1}= & g(\gamma)-4 \ln \frac{g^{\prime}(\gamma)}{2}-\frac{\gamma-\frac{1}{\gamma} \ln \left(\frac{g^{\prime}(\gamma)}{2}\right)^{5}+O\left(\frac{\ln \left(\frac{g^{\prime}(\gamma)}{2}\right)^{5}}{\gamma^{3}}\right)-r_{1}}{\frac{1}{\gamma}\left(1+\frac{1}{\gamma^{2}}\left[\frac{1}{2}, \frac{5}{2}\right]+O\left(\frac{\ln ^{2} \gamma}{\gamma^{3}}\right)\right)} \\
= & g(\gamma)-4 \ln \frac{g^{\prime}(\gamma)}{2}-\gamma\left(\gamma-\frac{5}{\gamma} \ln \left(\frac{g^{\prime}(\gamma)}{2}\right)+O\left(\frac{\ln ^{2} \gamma}{\gamma^{3}}\right)-r_{1}\right) \\
& \times\left(1-\frac{\left[\frac{1}{2}, \frac{5}{2}\right]}{\gamma^{2}}+O\left(\frac{\ln ^{2} \gamma}{\gamma^{3}}\right)\right) \\
= & g(\gamma)-\gamma^{2}+\ln \left(\frac{\left.g^{\prime} \gamma\right)}{2}+r_{1} \gamma+\left[\frac{1}{2}, \frac{5}{2}\right]+O\left(\frac{\ln ^{2} \gamma}{\gamma}\right)\right. \\
= & r_{1} \gamma+\left[\frac{1}{2}, \frac{5}{2}\right]-(a-1) \ln \gamma+\ln K+O\left(\frac{\ln ^{2} \gamma}{\gamma}\right) .
\end{aligned}
$$

\subsection{Demonstrações dos Teoremas}

Teorema 4.6. Suponha que $h: \mathbb{R} \rightarrow \mathbb{R}$ satisfaz as condições $\left(A_{1}\right)$ e $\left(A_{2}\right)$. Então o primeiro zero $T(\gamma)($ desde $+\infty)$ da solução $y_{\gamma}$ de (4.7) satisfaz:

i) Se $a>1$, então $\lim _{\gamma \rightarrow+\infty} T(\gamma)=-\infty$.

ii) Se $a=1$, então $\lim _{\gamma \rightarrow+\infty} T(\gamma) \in\left[\frac{1}{2}+\ln K, \frac{5}{2}+\ln K\right]$.

iii) Se $a<1$, então $\lim _{\gamma \rightarrow+\infty} T(\gamma)=+\infty$.

Demonstração. Escrevemos $O_{+}(1)=f_{0}$ se a função $f_{0}$ for limitada superiormente por uma constante positiva. Note que $f(y)$ é limitada superiormente em qualquer intervalo da forma $\left[0, y_{0}\right]$, pois basta considerar $O_{+}(1)=\sup _{0 \leq s \leq y_{0}}|f(s)|$. Assim integrando a equação (4.6), no intervalo $\left[t, t_{3}\right] \operatorname{com} t_{3}$ tal que $y\left(t_{3}\right) \leq y_{0} \leq r_{1}$, temos

$$
-\left(y^{\prime}\left(t_{3}\right)-y^{\prime}(t)\right)=-O_{+}(1)\left(e^{-t_{3}}-e^{-t}\right)
$$

Em particular para $t=t_{2}, \operatorname{com} t_{2}<t_{3}$

$$
-\left(y^{\prime}\left(t_{3}\right)-y^{\prime}\left(t_{2}\right)\right)=-O_{+}(1)\left(e^{-t_{3}}-e^{-t_{2}}\right) .
$$

Integrando a (4.33) com relação a $t$ em $\left[t_{2}, t_{3}\right]$, temos

$$
y^{\prime}\left(t_{3}\right)\left(t_{3}-t_{2}\right)-\left(y\left(t_{3}\right)-y\left(t_{2}\right)\right)=O_{+}(1)\left(e^{-t_{3}}\left(t_{3}-t_{2}\right)+e^{-t_{3}}-e^{-t_{2}}\right) .
$$


Então

$$
y\left(t_{3}\right)-y\left(t_{2}\right)=y^{\prime}\left(t_{3}\right)\left(t_{3}-t_{2}\right)+O_{+}(1)\left(e^{-t_{2}}-e^{-t_{3}}\left(1+t_{3}-t_{2}\right)\right) .
$$

Note que,

$$
O_{+}(1)\left(e^{-t_{2}}-e^{-t_{3}}\left(1+t_{3}-t_{2}\right)\right)=O_{+}(1) e^{-t_{2}}\left(1-e^{t_{2}-t_{3}}\left(1+t_{3}-t_{2}\right)\right)=O_{+}(1) e^{-t_{2}},
$$

pois $1-e^{t_{2}-t_{3}}\left(1+t_{3}-t_{2}\right) \leq 1$. Logo,

$$
y\left(t_{3}\right)-y\left(t_{2}\right)=y^{\prime}\left(t_{3}\right)\left(t_{3}-t_{2}\right)+O_{+}(1) e^{-t_{2}} .
$$

Note que, esta estimativa pode ser melhorada usando a condição $\left(A_{2}\right)$, pois

$$
f(s)=h(s) e^{s^{2}} \leq c K e^{r_{1}^{2}} s^{1+\sigma}, \quad \text { se } 0 \leq s \leq r_{1} .
$$

Desse modo, podemos considerar $O_{+}(1)=O\left(y_{0}^{1+\sigma}\right)$. E, portanto,

$$
y\left(t_{3}\right)-y\left(t_{2}\right)=y^{\prime}\left(t_{3}\right)\left(t_{3}-t_{2}\right)+O\left(y_{0}^{1+\sigma}\right) e^{-t_{2}} .
$$

Usando (4.35), com $t_{2}=T(\gamma)$ e $t_{3}=T_{1}$, temos

$$
y\left(T_{1}\right)-y(T(\gamma))=y^{\prime}\left(T_{1}\right)\left(T_{1}-T(\gamma)\right)+O_{+}(1) e^{-T(\gamma)}
$$

Lembrando que, $y\left(T_{1}\right)=r_{1}$ e $y(T(\gamma))=0$. Além disso, pelo Lema 4.4, temos $y^{\prime}\left(T_{1}\right)=\frac{1}{\gamma}+O\left(\frac{1}{\gamma^{3}}\right)$ e pelo Lema 4.5 , temos $T_{1}=r_{1} \gamma-(a-1) \ln \gamma+O(1)$. Substituindo em (4.37), temos

$$
\begin{aligned}
r_{1}= & \left(\frac{1}{\gamma}-O\left(\frac{1}{\gamma^{3}}\right)\right)\left(r_{1} \gamma-(a-1) \ln \gamma+O(1)-T(\gamma)\right)+O_{+}(1) e^{-T(\gamma)} \\
= & r_{1}-(a-1) \frac{\ln \gamma}{\gamma}-\frac{T(\gamma)}{\gamma}+\frac{O(1)}{\gamma}+O_{+}(1) e^{-T(\gamma)}+r_{1} O\left(\frac{1}{\gamma}\right) \\
& -(a-1) \ln \gamma O\left(\frac{1}{\gamma^{3}}\right)+O\left(\frac{1}{\gamma^{3}}\right)-T(\gamma) O\left(\frac{1}{\gamma^{3}}\right) \\
= & r_{1}-(a-1) \frac{\ln \gamma}{\gamma}-\frac{T(\gamma)}{\gamma}+\frac{O(1)}{\gamma}+O_{+}(1) e^{-T(\gamma)} .
\end{aligned}
$$

Assim,

$$
\frac{T(\gamma)}{\gamma}=-(a-1) \frac{\ln \gamma}{\gamma}+\frac{O(1)}{\gamma}+O_{+}(1) e^{-T(\gamma)}
$$

Portanto,

$$
T(\gamma)=-(a-1) \ln \gamma+O(1)+O_{+}(1) \gamma e^{-T(\gamma)}
$$


Prova de iii) Suponha que $\lim _{\gamma \rightarrow+\infty} T(\gamma) \neq+\infty$, então existe $c_{1} \in \mathbb{R}$ tal que $T(\gamma) \leq$ $c_{1}$, para todo $\gamma>0$.

Logo, usando (4.38), temos

$$
-(a-1) \ln \gamma+O(1)+O_{+}(1) \gamma e^{-T(\gamma)} \leq c_{1} .
$$

Como $e^{-c_{1}} \leq e^{-T(\gamma)}$, temos

$$
-(a-1) \ln \gamma+O(1)+O_{+}(1) \gamma e^{-c_{1}} \leq c_{1} .
$$

Fazendo $\gamma \rightarrow+\infty$ na desigualdade acima e dado que $a<1$, temos que $+\infty \leq c_{1}$, o qual é uma contradição. Portanto, se $a<1$, temos que $\lim _{\gamma \rightarrow+\infty} T(\gamma)=+\infty$.

Para provar $i$ ) e $i i)$ precisaremos das seguintes afirmações.

Afirmação 1: Existe $\gamma_{0}$ tal que

$$
T(\gamma)<4 \ln \gamma, \text { se } \gamma \geq \gamma_{0}
$$

além disso, se $a=1$, existe $c_{2}>0$, tal que $-c_{2} \leq T(\gamma)$, para todo $\gamma \geq \gamma_{0}$.

De fato, suponha por contradição que $4 \ln \gamma \leq T(\gamma)$, para todo $\gamma \in \mathbb{R}$. Portanto, $e^{-T(\gamma)} \leq e^{-4 \ln \gamma}=\frac{1}{\gamma^{4}}$ e , portanto, substituindo em (4.38), temos

$$
4 \ln \gamma \leq T(\gamma) \leq-(a-1) \ln \gamma+O(1)+O_{+}(1) \frac{1}{\gamma^{3}}
$$

Fazendo $\gamma \rightarrow+\infty$ e usando o fato que $a \geq 1$, temos uma contradição.

Portando, existe $\gamma_{0}$ tal que $T(\gamma)<4 \ln \gamma$, para todo $\gamma \geq \gamma_{0}$,

No caso que $a=1$ em (4.38), temos

$$
T(\gamma)=O(1)+O_{+}(1) \gamma e^{-T(\gamma)}
$$

Usando a primeira parte da Afirmação 1, temos $e^{-T(\gamma)} \geq e^{-4 \ln \gamma}=\frac{1}{\gamma^{4}}$, e assim

$$
T(\gamma) \geq O(1)+O_{+}(1) \frac{1}{\gamma}
$$

donde concluímos a prova.

Afirmação 2:

$$
y^{\prime}(4 \ln \gamma)=y^{\prime}\left(T_{1}\right)+O\left(\frac{1}{\gamma^{4}}\right)
$$


De fato, pelo Lema 4.5, $T_{1}=r_{1} \gamma-(a-1) \gamma+O(1)$. Logo, para $\gamma$ suficientemente grande, temos

$$
4 \ln \gamma<T_{1}=r_{1} \gamma-(a-1) \gamma+O(1)
$$

Usando (4.34), com $t_{3}=T_{1}$ e $t_{2}=4 \ln \gamma$, temos

$$
y^{\prime}\left(T_{1}\right)-y^{\prime}(4 \ln \gamma)=O_{+}(1)\left(e^{-T_{1}}-e^{-4 \ln \gamma}\right) .
$$

Logo,

$$
y^{\prime}\left(T_{1}\right)=y^{\prime}(4 \ln \gamma)+O_{+}(1) e^{-r_{1} \gamma+(a-1) \gamma+O(1)}+O\left(\frac{1}{\gamma^{4}}\right) .
$$

donde concluímos a prova, notando que $O_{+}(1) e^{-r_{1} \gamma+(a-1) \gamma+O(1)}=O\left(\frac{1}{\gamma^{4}}\right)$.

Afirmação 3:

$$
y(4 \ln \gamma)=\frac{4 \ln \gamma}{\gamma}-\frac{1}{\gamma}\left(\left[\frac{1}{2}, \frac{5}{2}\right]-(a-1) \ln \gamma+\ln K\right)+O\left(\frac{\ln ^{2}}{\gamma^{2} \gamma}\right)
$$

De fato, usando (4.35) com $t_{3}=T_{1}$ e $t_{2}=4 \ln \gamma$, temos

$$
y\left(T_{1}\right)-y(4 \ln \gamma)=y^{\prime}\left(T_{1}\right)\left(T_{1}-4 \ln \gamma\right)+O_{+}(1) e^{-4 \ln \gamma}
$$

Usando as estimativas dos Lemas 4.4 e 4.5, obtemos

$$
\begin{aligned}
r_{1}-y(4 \ln \gamma)= & \left(\frac{1}{\gamma}+O\left(\frac{1}{\gamma^{3}}\right)\right)\left(\left[\frac{1}{2}, \frac{5}{2}\right]+r_{1} \gamma-(a-1) \ln \gamma+\ln K+O\left(\frac{\ln ^{2} \gamma}{\gamma}\right)-4 \ln \gamma\right) \\
& +O(1) \frac{1}{\gamma^{4}} \\
= & r_{1}+\frac{1}{\gamma}\left(\left[\frac{1}{2}, \frac{5}{2}\right]-(a-1) \ln \gamma+\ln K\right)-\frac{4 \ln \gamma}{\gamma}+O\left(\frac{\ln ^{2} \gamma}{\gamma^{2}}\right) .
\end{aligned}
$$

Donde concluímos a prova da afirmação.

Usando (4.36) com $t_{3}=4 \ln \gamma$ e $t_{2}=T(\gamma)$, temos

$$
y(4 \ln \gamma)-y(T(\gamma))=y^{\prime}(4 \ln \gamma)(4 \ln \gamma-T(\gamma))+O\left(\left(\frac{\ln \gamma}{\gamma}\right)^{1+\gamma}\right) e^{-T(\gamma)}
$$

Usando as Afirmações 2 e 3, temos

$$
\frac{4 \ln \gamma}{\gamma}-\frac{1}{\gamma}\left(\left[\frac{1}{2}, \frac{5}{2}\right]+r_{1} \gamma-(a-1) \ln \gamma+\ln K\right)+O\left(\frac{\ln ^{2} \gamma}{\gamma}\right)
$$




$$
=\left(\frac{1}{\gamma}+O\left(\frac{1}{\gamma^{3}}\right)\right)(4 \ln \gamma-T(\gamma))+O\left(\left(\frac{\ln \gamma}{\gamma}\right)^{1+\sigma}\right) e^{-T(\gamma)}
$$

Portanto,

$$
T(\gamma)\left(1+O\left(\frac{1}{\gamma^{2}}\right)\right)=\left[\frac{1}{2}, \frac{5}{2}\right]+r_{1} \gamma-(a-1) \ln \gamma+\ln K+O\left(\frac{\ln ^{1+\sigma} \gamma}{\gamma^{\sigma}}\right) e^{-T(\gamma)}
$$

Prova de i) Suponha que $\lim _{\gamma \rightarrow+\infty} T(\gamma) \neq-\infty$, então existe uma constante $c_{3}>0$ tal que $T(\gamma) \geq-c_{3}$, para todo $\gamma \in \mathbb{R}$, logo, $e^{-T(\gamma)} \leq e^{c_{3}}$. Usando (4.39), temos

$$
T(\gamma)\left(1+O\left(\frac{1}{\gamma^{2}}\right)\right) \leq O(1)-(a-1) \ln \gamma+O\left(\frac{\ln ^{1+\sigma} \gamma}{\gamma^{\sigma}}\right) e^{c_{3}}
$$

Fazendo $\gamma \rightarrow+\infty$ e usando o fato que $a>1$ temos que $-c_{3} \leq-\infty$, o qual é uma contradição.

Prova de ii) Usando (4.39), para $a=1$, temos

$$
T(\gamma)\left(1+O\left(\frac{1}{\gamma^{2}}\right)\right)=\left[\frac{1}{2}, \frac{5}{2}\right]+r_{1} \gamma+\ln K+O\left(\frac{\ln ^{1+\sigma} \gamma}{\gamma^{\sigma}}\right) e^{-T(\gamma)}
$$

Da Afirmação 1, temos que $T(\gamma) \geq-c_{2}$ assim, $e^{-T(\gamma)} \leq e^{c_{2}}$.

Então fazendo $\gamma \rightarrow+\infty$, temos

$$
T(\gamma)=\left[\frac{1}{2}+\ln K, \frac{5}{2}+\ln K\right]
$$

Finalmente, provaremos o resultado principal deste capítulo.

Teorema 4.7. Suponha que $h \in \mathcal{C}^{2}(\mathbb{R})$ satisfaz as condições $\left(A_{1}\right)$ e $\left(A_{2}\right), \Omega=B_{1}(0)$ e $\alpha_{0}=1$ na equação (4.2). Então:

1) Se a $<$, então a equação (4.2) possui uma solução radial positiva.

2) Se $a=1$, então a equação (4.2) possui uma solução radial positiva para $K>K_{1}=\frac{4}{e^{1 / 2}}$, e existe uma constante $K_{0}$ com $0<K_{0} \leq K_{1}$ tal que a equação (4.2) não possui solução radial para $0<K<K_{0}$

3) Se $a>1$, existe $K_{2}$ tal que se $0<K \leq K_{2}$ então a equação (4.2) não possui solução radial e se $K>K_{2}$ então a equação (4.2) possui duas soluções radiais.

Demonstração. Iniciamos a prova do teorema com a seguinte afirmação. 
Afirmação $I$.

$$
\lim _{\gamma \rightarrow 0^{+}} T(\gamma)=-\infty
$$

De fato, usando (4.36) para $t_{2}=T(\gamma), t_{3}=+\infty$ e $y_{0}=\gamma \operatorname{com} \gamma \leq r_{1}$, temos

$$
\lim _{t \rightarrow+\infty} y(t)-y(T(\gamma))=\lim _{t \rightarrow+\infty} y^{\prime}(t)(t-T(\gamma))+O\left(\gamma^{1+\sigma}\right) e^{T(\gamma)}
$$

Portanto, $\gamma=\lim _{t \rightarrow+\infty} y^{\prime}(t) t+O\left(\gamma^{1+\sigma}\right) e^{T(\gamma)}, \operatorname{logo}$ usando a relações $t=-2 \ln \frac{r}{2}, y(t)=U(r)$, $U(1)=0$ e $U^{\prime}(0)=0$, tem-se $\lim _{t \rightarrow+\infty} y^{\prime}(t) t=0$, portanto $\gamma=O\left(\gamma^{1+\sigma}\right) e^{-T(\gamma)}$, então $e^{T(\gamma)}=O\left(\gamma^{\sigma}\right)$. Logo $\lim _{\gamma \rightarrow 0^{+}} e^{T(\gamma)}=\lim _{\gamma \rightarrow 0^{+}} O\left(\gamma^{\sigma}\right)=0$, donde temos a afirmação. tem-se

Como $t=-2 \ln \left(\frac{r}{2}\right)$ e $U(r)=y(t, \gamma)$, então $R(\gamma)=2 e^{-\frac{T(\gamma)}{2}}, \operatorname{logo}$ pela Afirmação $I$,

$$
\lim _{\gamma \rightarrow 0^{+}} R(\gamma)=+\infty
$$

a) Pela parte $i i i)$ do Teorema 4.6 temos que $\lim _{\gamma \rightarrow+\infty} T(\gamma)=+\infty$. Portanto

$$
\lim _{\gamma \rightarrow+\infty} R(\gamma)=\lim _{\gamma \rightarrow+\infty} 2 e^{-\frac{T(\gamma)}{2}}=0
$$

De (4.40) e do Lema 4.1, existe $\gamma_{0} \in \mathbb{R}$ tal que $R\left(\gamma_{0}\right)=1$. Portanto, $U\left(r, \gamma_{0}\right)$ é solução radial de (4.2).

b) Pelo Lema 4.1 para que exista solução do problema (4.2) é suficiente que

$$
\lim _{\gamma \rightarrow+\infty} R(\gamma)=\lim _{\gamma \rightarrow+\infty} 2 e^{-\frac{T(\gamma)}{2}}<1
$$

Isto quer dizer, que é necessário

$$
\lim _{\gamma \rightarrow+\infty} T(\gamma)>\ln 4
$$

Logo, pela parte $i$ ) do Teorema 4.6 , temos que é suficiente que $e^{1 / 2} K>4 \mathrm{e}$, portanto, existira solução do problema (4.2) se $K>K_{1}=\frac{4}{e^{1 / 2}}$.

Por outro lado, pela parte $i i)$ do Teorema 4.6, temos que $\lim _{\gamma \rightarrow+\infty} T(\gamma) \leq \frac{5}{2}+\ln K$, logo, existe uma constante $c>0$ tal que $T(\gamma) \leq c$, para todo $\gamma>0$ e, por tanto,

$$
R(\gamma)=2 e^{-\frac{T(\gamma)}{2}} \geq 2 e^{-\frac{c}{2}}, \quad \text { para todo } \gamma>0
$$

Note que, como $R$ depende também da constante $K$ nas condições $\left(A_{1}\right)$ e 
$\left(A_{2}\right)$ (assumindo que as demais constantes continuam fixas) Portanto, podemos escrever $R=R(\gamma, K)$.

Afirmação $I I$.

$$
R\left(\gamma, \frac{K}{d}\right)=\sqrt{d} R(\gamma, K), \quad \forall \gamma>0
$$

De fato, fazendo a mudança de variável $s=t-\ln d$ e seja $w(s)=u(t)$, temos

$$
-w^{\prime \prime}(s)=-y^{\prime \prime}(t)=h(y) e^{y^{2}-t}=h(w) e^{w^{2}-(s+\ln d)} .
$$

Assim,

$$
-w^{\prime \prime}=\frac{1}{d} h(w) e^{w^{2}-s}
$$

Seja $S(\gamma)$ o primeiro zero (desde $+\infty$ ) de $w(s) \operatorname{logo}, S(\gamma)=T(\gamma)-\ln d, \forall \gamma>0$, além disso $\frac{h(r)}{d}$ satisfaz as condições $\left(A_{1}\right)$ e $\left(A_{2}\right)$ com $\tilde{K}=\frac{K}{d}$ e, portanto,

$$
R\left(\gamma, \frac{K}{d}\right)=2 e^{-\frac{S(\gamma)}{2}}=2 e^{-\frac{T(\gamma)-\ln d}{2}}=\sqrt{d} R(\gamma, K)
$$

Escolha $d_{0}>\frac{e^{c}}{4} \operatorname{logo}$, de (4.41) e (4.42), temos

$$
R\left(\gamma, \frac{K}{d_{0}}\right)=\sqrt{d_{0}} R(\gamma, K)>\left(\frac{e^{c / 2}}{2}\right)\left(\frac{2}{e^{c / 2}}\right)=1
$$

Note que, para $d_{1}>d_{2}$,

$$
R\left(\gamma, \frac{K}{d_{1}}\right)=\sqrt{d_{1}} R(\gamma, K)>\sqrt{d_{2}} R(\gamma, K)=R\left(\gamma, \frac{K}{d_{2}}\right) .
$$

Em particular, temos que $R$ é uma função decrescente na segunda variável.

Logo, para cada $\tilde{K}$ tal que $0<\tilde{K} \leq K_{0}=\frac{K}{d_{0}}$, temos

$$
R(\gamma, \tilde{K})>1
$$

Isto, é que o primeiro zero de $U(r)$ é maior que 1 para todo $\gamma>0$, donde temos que não existe solução radial de (4.2) se $0<\tilde{K} \leq K_{0}=\frac{K}{d_{0}}$.

c) Neste caso, pela parte $i$ ) do Teorema 4.6, temos $\lim _{\gamma \rightarrow+\infty} T(\gamma)=-\infty$. Logo, para qualquer $K>0$ fixado, existe $c>0$, tal que $T(\gamma) \leq c$, para todo $\gamma>0$.

Considere $K=1$ e seja $c_{1}=\sup _{\gamma>0} T(\gamma), \log$ como na parte $b$ ) existe $d_{1}=\frac{e^{c_{1}}}{4}$ tal 
que

$$
R\left(\gamma, \frac{1}{d_{1}}\right) \geq 1, \quad \forall \gamma>0
$$

Seja $K_{2}=\frac{1}{d_{1}}$, como $R$ é decrescente na segunda variável, então

$$
R(\gamma, K)>1, \quad \text { para todo } \gamma>0, \quad \text { se } 0<K<K_{2}
$$

Portanto, não existe solução radial de (4.2).

Por outro lado, como $c_{1}=\sup _{\gamma>0} T(\gamma, 1)$ e $R(\gamma, K)=2 e^{-\frac{T(\gamma, K)}{2}}$, temos

$$
\min _{\gamma>0} R(\gamma, 1)=\frac{2}{e^{c_{1} / 2}}=\frac{1}{\sqrt{d_{1}}}=\sqrt{K_{2}}
$$

Logo, se $K>K_{2}$

$$
\min _{\gamma>0} R(\gamma, K)=\min _{\gamma>0} R(\gamma, 1) \frac{1}{\sqrt{K}}=\sqrt{\frac{K_{2}}{K}}<1 .
$$

Seja $\gamma_{0}>0$ tal que $R\left(\gamma_{0}, K\right)<1$. Além disso de (4.40) e $\lim _{\gamma \rightarrow+\infty} R(\gamma)=+\infty$, existem $\gamma_{1}<\gamma_{0}<\gamma_{2}$ tais que $R\left(\gamma_{1}, K\right)>1$ e $R\left(\gamma_{2}, K\right)>1$, usando o Lema 4.1, existem $\gamma_{3}<\gamma_{0}<\gamma_{4}$ tais que

$$
R\left(\gamma_{3}, K\right)=R\left(\gamma_{4}, K\right)=1
$$

Isto é, temos duas soluções radias para o problema (4.2). 


\section{Apêndice}

\section{$\mathcal{A}$}

\section{Resultados Básicos}

Neste apêndice são apresentados resultados básicos que são utilizados no desenvolvimento deste trabalho. Embora sejam omitidas as demonstração desses resultados, são citadas referências que as contém.

Teorema A.1. (Desigualdade de Hölder) [5, Theorem 4.6] Sejam $\Omega$ um domínio de $\mathbb{R}^{N}$, $1 \leq p \leq \infty$ e $1 \leq q \leq \infty \operatorname{com} \frac{1}{p}+\frac{1}{q}=1$. Se $f \in L^{p}(\Omega)$ e $g \in L^{q}(\Omega)$, então

$$
f g \in L^{1}(\Omega) \quad e \quad \int_{\Omega}|f(x) g(x)| d x \leq\|f\|_{L^{p}(\Omega)}\|g\|_{L^{q}(\Omega)} .
$$

Teorema A.2. (Teorema da Convergência Dominada de Lebesgue) [5, Theorem 4.2] Seja $\left(f_{n}\right)$ uma sequência de funções em $L^{1}(\Omega)$ que satisfaz:

(i) $f_{n}(x) \longrightarrow f(x)$ q.t.p.em $\Omega$,

(ii) Existe uma função $h \in L^{1}(\Omega)$, tal que para todo $n \geq 1$, verifica-se $\left|f_{n}(x)\right| \leq h(x)$ q.t.p. em $\Omega$.

Então,

$$
f \in L^{1}(\Omega) \quad \text { e }\left\|f_{n}-f\right\|_{L^{1}(\Omega)} \longrightarrow 0
$$

Teorema A.3. [5, Theorem 4.9] Sejam $1 \leq p \leq \infty,\left\{u_{n}\right\}$ uma sequência em $L^{p}(\Omega) e$ $u \in L^{p}(\Omega)$, tais que $u_{n} \longrightarrow u$ em $L^{p}(\Omega)$. Então, existe uma subsequência $\left\{u_{n_{k}}\right\} \subseteq\left\{u_{n}\right\}$, satisfazendo: $u_{n_{k}}(x) \longrightarrow u(x)$ q.t.p. em $\Omega$, 


\section{A.1 Os Espaços de Sobolev}

Definição A.4. Sejam $u, v \in L_{L o c}^{1}(\Omega)$ e $\alpha=\left(\alpha_{1}, \alpha_{2}, \ldots, \alpha_{n}\right)$ um multi-índice de ordem $|\alpha|=\alpha_{1}+\ldots+\alpha_{n}$. Dizemos que vé $\alpha$-derivada fraca de u e escrevemos $D^{\alpha} u=v$, desde que

$$
\int_{\Omega} u D^{\alpha} \varphi d x=(-1)^{|\alpha|} \int_{\Omega} v \varphi d x, \quad \forall \varphi \in C_{c}^{\infty}(\Omega)
$$

$\operatorname{com} D^{\alpha} \varphi=\frac{\partial^{\alpha_{1}}}{\partial x_{1}^{\alpha_{1}}} \cdots \frac{\partial^{\alpha_{n}}}{\partial x_{n}^{\alpha_{n}}} \varphi$.

Definição A.5. Sejam $p \in[1, \infty], k$ um inteiro positivo e $\Omega$ um aberto não vazio de $\mathbb{R}^{n}$. Definimos o espaço de Sobolev $W^{k, p}(\Omega)$ como

$$
W^{k, p}(\Omega)=\left\{u \in L^{p}(\Omega) ; D^{\alpha} u \in L^{p}(\Omega), \quad \forall \alpha \in \mathbb{N}^{N} \operatorname{com}|\alpha| \leq k\right\}
$$

Observe que na definição de $W^{k, p}(\Omega)$ a derivada considerada é a derivada fraca. Assim, dizer que $D^{\alpha} u \in L^{p}(\Omega)$ significa que existe $v_{\alpha} \in L^{p}(\Omega)$ tal que $D^{\alpha} u=v_{\alpha}$ no sentido fraco.

Definição A.6. Se $u \in W^{k, p}(\Omega)$ definimos sua norma como sendo

$$
\|u\|_{W^{k, p}(\Omega)}:=\left\{\begin{array}{cl}
\left(\sum_{|\alpha| \leq k} \int_{\Omega}\left|D^{\alpha} u\right|^{p} d x\right)^{1 / p}, & 1 \leq p<\infty \\
\sum_{|\alpha| \leq k}\left\|D^{\alpha} u\right\|_{L^{\infty}(\Omega)} & , \quad p=\infty .
\end{array}\right.
$$

Se $p=2$ e $k=1$ escreveremos

$$
H^{1}(\Omega)=W^{1,2}(\Omega)
$$

Teorema A.7. (Desigualdade geral de Sobolev) [1, Theorem 4.12] Seja $\Omega \subseteq \mathbb{R}^{N}$ um aberto limitado regular. Então as imersões abaixo são contínuas:

(i) Se $k p<N$ então $W^{k, p}(\Omega) \hookrightarrow L^{q}(\Omega)$, para todo $1 \leq q \leq \frac{p N}{N-k p}$.

(ii) Se $k p=N$ e $p>1$ então $W^{k, p}(\Omega) \hookrightarrow L^{q}(\Omega)$, para todo $1 \leq q<\infty$.

Teorema A.8. (Imersões Compactas: Rellich-Kondrachov) [1, Theorem 6.3] Seja $\Omega \subseteq \mathbb{R}^{N}$ um aberto limitado regular. Então, as seguintes imersões são compactas:

(i) Se $k p<N$ então $W^{k, p}(\Omega) \hookrightarrow L^{q}(\Omega)$ para todo $1 \leq q<\frac{p N}{N-k p}$. 
(ii) Se $k p=N$ e $p>1$ então $W^{k, p}(\Omega) \hookrightarrow L^{q}(\Omega)$ para todo $1 \leq q<\infty$.

\section{A.2 O Teorema do Passo da Montanha}

Uma das principais ferramentas empregadas para estudar a existência de soluções de problemas elípticos semilineares é a teoria de pontos críticos. Um dos resultados principais desta teoria é o Teorema do Passo da Montanha de Ambrosetti e Rabinowitz. Este teorema garante, sob hipóteses adequadas, a existência de pontos críticos minimax para funcionais $\phi: X \rightarrow \mathbb{R}$ onde $X$ é um espaço de Banach real. O Teorema do Passo da Montanha envolve uma condição técnica, a condição de Palais-Smale.

Definição A.9. Seja $\varphi: U \rightarrow \mathbb{R}$ onde $U$ é um subconjunto aberto de um espaço de Banach $X$ e $u \in U$. Dizemos que $\varphi$ é Gâteaux-diferenciável em u se existe $A \in X^{*}$, tal que

$$
\lim _{t \rightarrow 0} \frac{\varphi(u+t v)-\varphi(u)}{t}=A v, \quad \forall v \in X
$$

O operador A é chamado a derivada de Gâteaux de $\varphi$ em u e é denotado por $\varphi^{\prime}(u)$.

Definição A.10. Seja $\varphi: U \rightarrow \mathbb{R}$ onde $U$ é um subconjunto aberto de um espaço de Banach $X$ e $u \in U$. Dizemos que $\varphi$ é Fréchet-diferenciável em u se existe $A \in X^{*}$, tal que

$$
\lim _{h \rightarrow 0} \frac{|\varphi(u+h)-\varphi(u)-A h|}{\|h\|}=0 .
$$

O operador A é chamado a derivada de Fréchet de $\varphi$ em u e é denotado por $D \varphi(u)$.

Observação: operador adjunto. Sejam $E$ e $F$ espaços normados e $T: E \rightarrow F$ um operador linear contínuo. O operador adjunto de $T$, representado por $T^{*}$ é o operador $T^{*}: F^{*} \rightarrow E^{*}$ definido por $T^{*} \phi:=\phi \circ T$ para $\phi \in F^{*}$. Tem-se então que $T^{*}$ é também um operador linear contínuo.

Proposição A.11. [14, Proposition 1.3] Suponha que $\varphi: U \rightarrow \mathbb{R}$ tenha derivada de Gâteaux em todo ponto de $U$ e que $\varphi^{\prime}: U \rightarrow X^{*}$ seja contínua (isto é, $\varphi$ tem derivada de Gâteaux contínua em $U$ ). Então $\varphi$ é Fréchet-diferenciável em $U$ e $D \varphi=\varphi^{\prime}$. Em particular, $D \varphi: U \rightarrow X^{*}$ é contínua, isto é, $\varphi$ tem derivada de Fréchet contínua em $U$ (o que é denotado $\left.\varphi \in C^{1}(U, \mathbb{R})\right)$.

Definição A.12. Seja $X$ um espaço de Banach real e $\varphi \in C^{1}(X, \mathbb{R})$ um funcional. Diz-se que $\phi$ satisfaz a condição de Palais-Smale no nível $c \in \mathbb{R}$ (denotada por $(P S)_{c}$ ) se toda sequência $\left(u_{n}\right) \subseteq X$ para a qual $\phi\left(u_{n}\right) \longrightarrow$ ce $\phi^{\prime}\left(u_{n}\right) \longrightarrow 0$, quando $n \rightarrow \infty$, possui uma subsequência convergente. Diz-se que $\phi$ satisfaz a condição (PS) quando $\phi$ satisfaz $(P S)_{c}$ para todo $c \in \mathbb{R}$. 
Teorema A.13. (Teorema do Passo da Montanha) [4] Sejam X um espaço de Banach e $\phi \in C^{1}(X, \mathbb{R})$ um funcional. Suponha que $\phi$ satisfaz a condição $(P S), \phi(0)=0 e$

i) existem $a>0$ e $\rho>0$, tais que

$$
\phi(u) \geq a, \quad \text { se }\|u\|=\rho, u \in W
$$

ii) existe $e \in X,\|e\|>\rho$, tal que $\phi(e) \leq 0$.

Então $\phi$ possui um valor crítico $c \geq$ a que pode ser caracterizado como

$$
c=\inf _{\gamma \in \Gamma} \sup _{t \in[0,1]} \phi(\gamma(t))
$$

onde,

$$
\Gamma=\{\gamma \in C([0,1], X) ; \gamma(0)=0 \text { e } \gamma(1)=e\}
$$

Teorema A.14. [4] Sejam $X$ um espaço de Banach de dimensão infinita com $X=V \oplus W$, $\operatorname{dim}(V)<+\infty$ e $\phi \in C^{1}(X, \mathbb{R})$ é um funcional par, $\phi(0)=0$ e satisfaz a condição (PS). Além disso, suponha que $\phi$ satisfaz

$\left.i^{\prime}\right)$ existem $a>0$ e $\rho>0$ tais que:

$$
\phi(u) \geq a, \quad \text { se } u \in W e\|u\|=\rho,
$$

ii') para cada subespaço $\tilde{X}$ de $X$ de dimensão finita, existe $R=R(\tilde{X})>0$ tal que $\phi(u) \leq 0$ se $\|u\|>R$ e $u \in \tilde{X}$.

Então ф possui uma infinidade de valores críticos.

Teorema A.15. (Teorema do Passo da Montanha Generalizado) [4] Seja X um espaço de Banach de dimensão infinita, com $X=V \oplus W, \operatorname{dim}(V)<+\infty$. Suponha $\phi \in \mathcal{C}^{1}(X, \mathbb{R})$ um funcional satisfazendo a condição (PS) $e$

i) existem $a>0$ e $\rho>0$ tais que $\phi(u) \geq a$, se $\|u\|=\rho, u \in W$,

ii) existe $e \in W,\|e\|=1$, e existe $R>\rho$, tal que se

$$
Q=\left(\overline{B_{R}} \cap V\right) \oplus\{r e: 0<r<R\}
$$

Então,

$$
\phi(u) \leq 0, \quad \forall u \in \partial Q
$$


Então $\phi$ possui um valor crítico em $c \geq a$, com

$$
c=\inf _{h \in \Gamma} \sup _{u \in \bar{Q}} \phi(h(u))
$$

onde,

$$
\Gamma=\{h \in \mathcal{C}(\bar{Q}, X) ; h=I d, \text { em } \partial Q\} .
$$


Apêndice

$\mathcal{B}$

\section{Resultados técnicos}

Nesta parte do apêndice estão apresentados alguns resultados técnicos utilizados nesta dissertação.

Lema B.1. Sejam $\xi \in \mathbb{R}^{N} e \Omega \subset \mathbb{R}^{N}$ tais que $|\Omega|<\infty$. Se $0 \leq k<N$, então

$$
\int_{\Omega} \frac{1}{|x-\xi|^{k}} d x \leq C(k, N) \frac{|\Omega|^{1-\frac{k}{N}}}{N-k}
$$

Demonstração. Seja $B_{r}(\xi) \subset \mathbb{R}^{N}$ a bola tal que $\left|B_{r}(\xi)\right|=|\Omega|$. Note que, para cada $x \in \Omega \backslash B_{r}(\xi)$ e $y \in B_{r}(\xi) \backslash \Omega$, temos $\frac{1}{|x-\xi|^{k}} \leq \frac{1}{|y-\xi|^{k}}$ e como $\left|\Omega \backslash B_{r}(\xi)\right|=\left|B_{r}(\xi) \backslash \Omega\right|$, segue-se que

$$
\int_{\Omega \backslash B_{r}(\xi)} \frac{1}{|x-\xi|^{k}} d x \leq \int_{B_{r}(\xi) \backslash \Omega} \frac{1}{|y-\xi|^{k}} d y .
$$

Logo,

$$
\begin{aligned}
\int_{\Omega} \frac{1}{|x-\xi|^{k}} d x & =\int_{\Omega \cap B_{r}(\xi)} \frac{1}{|x-\xi|^{k}} d x+\int_{\Omega \backslash B_{r}(\xi)} \frac{1}{|x-\xi|^{k}} d x \\
& \leq \int_{\Omega \cap B_{r}(\xi)} \frac{1}{|y-\xi|^{k}} d y+\int_{B_{r}(\xi) \backslash \Omega} \frac{1}{|y-\xi|^{k}} d y \\
& =\int_{B_{r}(\xi)} \frac{1}{|y-\xi|^{k}} d y=\frac{\omega_{N} r^{N-k}}{N-k} .
\end{aligned}
$$

Onde $\omega_{N}$ é a medida da bola unitária dimensional. Como $\omega_{N} r^{N}=\left|B_{r}(\xi)\right|=|\Omega|$. E, portanto,

$$
\int_{\Omega} \frac{1}{|x-\xi|^{k}} d x \leq \omega_{N}^{k / N} \frac{|\Omega|^{1-k / N}}{N-k}
$$


Lema B.2. A aplicação:

$$
\begin{aligned}
\gamma: L^{1}(\Omega) & \rightarrow L^{1}(\Omega) \\
f & \mapsto \gamma(f)(x)=\int_{\Omega} \frac{|f(\xi)|}{|x-\xi|^{N-1}} d \xi
\end{aligned}
$$

é lipschitziana e, portanto, contínua.

Demonstração. Do Lema B.1, existe uma constante $C_{0}>0$, tal que para todo $\xi \in \Omega$, tem-se

$$
\int_{\Omega} \frac{1}{|x-\xi|^{N-1}} d x \leq C_{0}|\Omega|^{\frac{1}{N}}
$$

Dados $f, g \in L^{1}(\Omega)$, temos

$$
\begin{aligned}
\|\gamma(f)-\gamma(g)\|_{L^{1}(\Omega)} & \leq \int_{\Omega} \int_{\Omega} \frac{|f(\xi)-g(\xi)|}{|x-\xi|^{N-1}} d \xi d x=\int_{\Omega}|f(\xi)-g(\xi)| \int_{\Omega} \frac{1}{|x-\xi|^{N-1}} d x d \xi \\
& \leq C_{0}|\Omega|^{\frac{1}{N}} \int_{\Omega}|f(\xi)-g(\xi)| d \xi=C_{0}|\Omega|^{\frac{1}{N}}\|f-g\|_{L^{1}(\Omega)},
\end{aligned}
$$

de onde segue o lema.

Lema B.3. Dado $n \geq 2$, tem-se as seguintes desigualdades

(i) $(a+b)^{n} \geq a^{n}+n a^{n-1} b+(n-1) a^{n-2} b^{2}$, se $a \geq 0, a+b \geq 0$.

(ii) $a^{n}+n a^{n-1} b+b^{n} \leq(a+b)^{n} \leq a^{n}+C_{7}\left(a^{n-1} b+b^{n}\right)$, se $a, b \geq 0, C_{7}=C_{7}(n)$.

Demonstração. Usando a identidade $F(1)=F(0)+F^{\prime}(0)+\int_{0}^{1} F^{\prime}(x)(1-x) d x$, para $F(x)=(a+b x)^{n}$, temos

$$
(a+b)^{n}=a^{n}+n a^{n-1} b+n(n-1) b^{2} \int_{0}^{1}(a+b x)^{n-2}(1-x) d x .
$$

(i) Note que, se $a+b \geq 0$ e $0 \leq x \leq 1$, então $a+x b=(1-x) a+x(a+b) \geq(1-x) a$, como $a \geq 0$, temos

$$
\int_{0}^{1}(a+b x)^{n-2}(1-x) d x \geq a^{n-2} \int_{0}^{1}(1-x)^{n-1} d x \geq \frac{a^{n-2}}{n} \geq 0 .
$$

Substituindo em (B.1) temos a desigualdade (i). 
(ii) Se $a \geq 0$ e $b \geq 0$, então $a+x b \geq 0$, $\log 0$

$$
\int_{0}^{1}(a+b x)^{n-2}(1-x) d x \geq b^{n-2} \int_{0}^{1} x^{n-2}(1-x) d x=\frac{b^{n-2}}{n(n-1)} .
$$

Substituindo em (B.1) temos a primeira desigualdade de (ii).

Note que, a segunda desigualdade de (ii) é valida para $a=0$, por outro lado, para cada $z>0$

$$
\frac{(1+z)^{n}-1}{z+z^{n}}=\sum_{j=1}^{n}\left(\begin{array}{l}
n \\
j
\end{array}\right) \frac{z^{j}}{z+z^{n}} \leq \sum_{j=1}^{n}\left(\begin{array}{l}
n \\
j
\end{array}\right)=2^{n}-1=C_{7}(n) .
$$

E, portanto, $(1+z)^{n} \leq 1+C_{7}\left(z+z^{n}\right)$, para todo $z>0$, então basta tomar $z=\frac{b}{a}$, no caso $a \neq 0$ para obter a segunda desigualdade de (ii).

Lema B.4. Seja $\Omega \subset \mathbb{R}^{2}$ limitado e $\left\{u_{n}\right\}$ uma sequência em $L^{1}(\Omega)$ tal que $u_{n} \rightarrow$ u em $L^{1}(\Omega)$ e suponha que as sequências $\left\{f\left(x, u_{n}\right)\right\}$ e $\{f(x, u)\}$ estão em $L^{1}(\Omega)$ para a função contínua $f: \Omega \times \mathbb{R} \rightarrow \mathbb{R}$. Além disso, suponha que existe $C>0$ tal que $\int_{\Omega}\left|f\left(x, u_{n}\right) u_{n}\right| d x \leq C$. Então $f\left(x, u_{n}\right) \rightarrow f(x, u), \quad$ em $L^{1}(\Omega)$.

Demonstração. Tome $\epsilon>0$ arbitrário, como $f(x, u) \in L^{1}(\Omega)$, existe $\delta>0$, tal que

$$
\int_{A}|f(x, u)| d x<\epsilon, \quad \text { se }|A|<\delta
$$

para qualquer conjunto $A$ mensurável em $\Omega$.

Defina $\Omega_{n}=\{x \in \Omega:|u(x)| \geq n\}$, como $u \in L^{1}(\Omega)$, então

$$
n\left|\Omega_{n}\right|=\int_{\Omega_{n}} n d x \leq \int_{\Omega_{n}}|u(x)| d x \leq \int_{\Omega}|u(x)| d x<+\infty .
$$

Donde $\lim _{n \rightarrow \infty}\left|\Omega_{n}\right|=0$, então existe $M_{1}>0$ tal que

$$
\left|\left\{x \in \Omega:|u(x)|>M_{1}\right\}\right|<\delta .
$$


Logo, para $M=\max \left\{M_{1}, \frac{C}{\epsilon}\right\}$

$$
\begin{aligned}
\left|\int_{\Omega}\right| f\left(x, u_{n}\right)|-| f(x, u)|d x|= & \left|\int_{\left\{\left|u_{n}\right| \geq M\right\}}\right| f\left(x, u_{n}\right)\left|d x+\int_{\left\{\left|u_{n}\right|<M\right\}}\right| f\left(x, u_{n}\right) \mid d x \\
& -\int_{\{|u| \geq M\}}|f(x, u)| d x-\int_{\{|u|<M\}}|f(x, u)| d x \mid \\
\leq & \int_{\left\{\left|u_{n}\right| \geq M\right\}}\left|f\left(x, u_{n}\right)\right| d x+\int_{\{|u| \geq M\}}|f(x, u)| d x \\
& +\left|\int_{\left\{\left|u_{n}\right|<M\right\}}\right| f\left(x, u_{n}\right)\left|d x-\int_{\{|u|<M\}}\right| f(x, u)|d x| .
\end{aligned}
$$

Analisaremos cada termo do lado direito

$$
\begin{aligned}
\int_{\left\{\left|u_{n}\right| \geq M\right\}}\left|f\left(x, u_{n}\right)\right| d x & =\int_{\left\{\left|u_{n}\right| \geq M\right\}} \frac{\left|f\left(x, u_{n}\right) u_{n}\right|}{\left|u_{n}\right|} d x \leq \frac{1}{M} \int_{\left\{\left|u_{n}\right| \geq M\right\}}\left|f\left(x, u_{n}\right) u_{n}\right| d x \\
& \leq \frac{1}{M} \int_{\Omega}\left|f\left(x, u_{n}\right) u_{n}\right| d x \leq \frac{C}{M} \leq \epsilon
\end{aligned}
$$

Dado que $|\{x \in \Omega:|u(x)| \geq M\}|=|\{x \in \Omega:|u(x)| \geq M\}|<\delta$, então por (B.2), temos

$$
\int_{\{|u| \geq M\}}|f(x, u)| d x<\epsilon .
$$

Seja $I_{n}=\left|\int_{\{|u|<M\}}\right| f\left(x, u_{n}\right)\left|d x-\int_{\{|u|<M\}}\right| f(x, u)|d x|$. Logo,

$$
\begin{aligned}
I_{n}= & \mid \int_{\Omega}\left(\left|f\left(x, u_{n}\right)\right|-|f(x, u)|\right) \chi_{\left\{\left|u_{n}\right|<M\right\}} d x \\
& +\int_{\Omega}|f(x, u)|\left(\chi_{\left\{\left|u_{n}\right|<M\right\}}-\chi_{\{|u|<M\}}\right) d x \mid \\
\leq & \int_{\Omega}\left|f\left(x, u_{n}\right)-f(x, u)\right| \chi_{\left\{\left|u_{n}\right|<M\right\}} d x+\int_{\Omega}|f(x, u)| \chi_{\{|u|>M\}} d x .
\end{aligned}
$$

Agora estimemos essas duas últimas integrais. Seja $h_{n}(x)=\left|f\left(x, u_{n}\right)-f(x, u)\right| \chi_{\left\{\left|u_{n}\right|<M\right\}}$. Observe que

$$
h_{n}(x) \rightarrow 0 \text {, q.t.p. em } \Omega \text {. }
$$

Também,

$$
\left|h_{n}(x)\right| \leq\left|f\left(x, u_{n}\right)\right| \chi_{\left\{\left|u_{n}\right|<M\right\}}+|f(x, u)| \chi_{\left\{\left|u_{n}\right|<M\right\}} \leq \bar{C}+|f(x, u)| \in L^{1}(\Omega),
$$


onde $\bar{C}=\sup \{f(x, t):(x, t) \in \bar{\Omega} \times[0, M]\}$. Pelo Teorema da Convergência Dominada, temos $\int_{\Omega} h_{n}(x) d x \rightarrow 0$ se $n \rightarrow \infty$, assim, existe $n_{0} \in \mathbb{N}$ tal que $0 \leq \int_{\Omega} \mid f\left(x, u_{n}\right)-$ $f(x, u) \mid \chi_{\left\{\left|u_{n}\right|<M\right\}} d x<\epsilon$, para todo $n \geq n_{0}$ e de (B.2), temos $\int_{\{|u|>M\}}|f(x, u)| d x<\epsilon$. E, portanto,

$$
\left|\int_{\left\{\left|u_{n}\right|<M\right\}}\right| f\left(x, u_{n}\right)\left|d x-\int_{\{|u|<M\}}\right| f(x, u)|d x|<2 \epsilon \quad \text { para todo } n \geq n_{0} .
$$

Logo,

$$
\left|\int_{\Omega}\right| f\left(x, u_{n}\right)|-| f(x, u)|d x|<4 \epsilon, \quad \text { para todo } n \geq n_{0} .
$$

E, por conseguinte,

$$
\int_{\Omega}\left|f\left(x, u_{n}\right)\right| d x \rightarrow \int_{\Omega}|f(x, u)| d x, \quad \text { quando } n \rightarrow \infty .
$$

Lema B.5. $\lim _{m \rightarrow \infty} m \int_{0}^{1} e^{\left(s^{2}-s\right) m} d s=2$.

Demonstração. Primeiramente provemos as seguintes afirmações

Afirmação 1. Para todo $0<b \leq \frac{1}{2}$

$$
\int_{0}^{b} e^{m\left(s^{2}-s\right)} d s=\int_{1-b}^{1} e^{m\left(s^{2}-s\right)} d s
$$

De fato, basta considerar a mudança de variável $t=1-s$.

Afirmação 2. Para todo $0<a<\frac{1}{2}$

$$
\lim _{m \rightarrow \infty} m \int_{a}^{1-a} e^{\left(s^{2}-s\right) m} d s=0
$$

De fato, observe que para $a \leq s \leq 1-a$ tem-se, $s^{2}-s \leq a^{2}-a<0$, logo,

$$
\lim _{m \rightarrow \infty} m \int_{a}^{1-a} e^{\left(s^{2}-s\right) m} d s \leq \lim _{m \rightarrow \infty} m e^{\left(a^{2}-a\right) m}(1-2 a)=0 .
$$

Afirmação 3. Para todo $0<b<1$

$$
\limsup _{m \rightarrow \infty} \int_{b}^{1} m e^{m\left(s^{2}-s\right)} \leq \frac{1}{b}
$$


De fato, como $b \leq s \leq 1$, então $s(s-1) \leq b(s-1)$, logo

$$
m \int_{b}^{1} e^{m\left(s^{2}-s\right)} d s \leq m \int_{b}^{1} e^{m b(s-1)} d s=\left.\frac{1}{b} e^{m b(s-1)}\right|_{b} ^{1}=\frac{1}{b}-\frac{e^{m\left(b^{2}-b\right)}}{b} .
$$

Então tomando limite superior tem-se a afirmação.

Note que, como $0 \leq s \leq 1$, então $s^{2}-s=s(s-1) \geq s-1$, pela Afirmação 1 , com $b=\frac{1}{2}$

$$
m \int_{0}^{1} e^{m\left(s^{2}-s\right)} d s=2 m \int_{\frac{1}{2}}^{1} e^{m\left(s^{2}-s\right)} d s \geq 2 m \int_{\frac{1}{2}}^{1} e^{m(s-1)} d s=\left.2 e^{m(s-1)}\right|_{\frac{1}{2}} ^{1}=2-2 e^{-\frac{m}{2}} .
$$

Logo, tomando limite inferior, temos

$$
\liminf _{m \rightarrow \infty} m \int_{0}^{1} e^{\left(s^{2}-s\right) m} d s \geq 2
$$

Por outro lado, seja $a=\frac{1}{n}$ e $b=1-a$, pela Afirmação 1 , tem-se

$$
m \int_{0}^{1} e^{m\left(s^{2}-s\right)} d s=m \int_{\frac{1}{n}}^{1-\frac{1}{n}} e^{m\left(s^{2}-s\right)} d s+2 m \int_{1-\frac{1}{n}}^{1} e^{m\left(s^{2}-s\right)} d s .
$$

Agora, tomando limite superior e usando a afirmações 2 e 3

$$
\limsup _{m \rightarrow \infty} \int_{0}^{1} m e^{m\left(s^{2}-s\right)} d s \leq \frac{2}{1-\frac{1}{n}}
$$

Logo, tomando o limite, quando $n \rightarrow \infty$, temos

$$
\limsup _{m \rightarrow \infty} m \int_{0}^{1} e^{m\left(s^{2}-s\right)} d s \leq 2 .
$$

A proposição segue-se de (B.3) e (B.4). 


\section{Referências Bibliográficas}

[1] ADAMS, R. A., FOURNIER, J. J. F. Sobolev spaces. Amsterdam: Academic Press, 2005.

[2] ADIMURTHI. Existence of positive solutions of the semilinear Dirichlet problems with critical growth for the $N$-Laplacian. Ann. Sc. Norm. Sup. Pisa, 17, p. 393-413, 1990.

[3] ADIMURTHI, SRIKANTH, P.N., YADAVA, S.L. Phenomena of critical exponent in $\mathbb{R}^{2}$. Proc. Royal Soc. Edinburgh Sect. A, 119, p. 19-25, 1991.

[4] AMBROSETTI, A., RABINOWITZ, P.H. Dual variational methods in critical point theory and applications. J. Funct. Anal., 14, p. 349-381, 1973.

[5] BREZIS. H. Functional analysis, Sobolev spaces and partial differential equations. New Yorh: Springer, 2010. (Série Universitext).

[6] CARLESON, L.; CHANG, A. On the existence of an extremal function for an inequality of J. Moser. Bull. Sc. Math., 110, p. 113-127, 1986.

[7] DE FIGUEIREDO, G.; MIYAGAKI, O.H.; RUF, B. Elliptic equations in $\mathbb{R}^{2}$ with nonlinearities in the critical growth range. Calc. Var. Partial Differential Equations, 3, p. 139-153, 1995.

[8] DE FIGUEIREDO, G.; RUF, B. On the existence and non-existence of solutions for elliptic equations with critical growth in $\mathbb{R}^{2}$. Comm. Pure Appl. Math., 48, p. 640-655, 1995.

[9] GIDAS, B.; NI, W.M.; NIRENBERG, L. Symmetry and related properties via maximum principle. Comm. Math. Phys., 68, p. 209-243, 1979. 
[10] KRASNOSELSKI, M . A., RUTICKII, Y. B. Convex functions and Orlicz Spaces. Groningen: P. Noord- hoff LTD., 1961.

[11] MOSER, J. A sharp form of an inequality by N. Trudinger. Indiana Univ. Math. J., 20, p. 1077-1092, 1971.

[12] PÓLYA, G.; SZEGÖ, G. Isoperimetric Inequalities in Mathematics Physics. Princeton: University Press, 1951.

[13] TRUDINGER, N. On embedding into Orlicz spaces and some applications. J. Math. Mech., 17, p. 473-484, 1967.

[14] WILLEM, M. Minimax Theorems. Boston: Birhauser, 1996. 